\title{
Blue emitting organic semiconductors under high pressure: status and outlook
}

\author{
Matti Knaapila $^{1^{*}}$ and Suchismita Guha ${ }^{2 * *}$ \\ ${ }^{1}$ Department of Physics, Technical University of Denmark, 2800 Kgs. Lyngby, Denmark \\ ${ }^{2}$ Department of Physics and Astronomy, University of Missouri, Columbia, MO 65211, USA \\ *E-mail: matti.knaapila@fysik.dtu.dk \\ ** E-mail: guhas@missouri.edu \\ Received XXX \\ Published XXX \\ This article was invited by $\mathrm{XX}$
}

\begin{abstract}
This review describes essential optical and emerging structural experiments that use high GPa range hydrostatic pressure to probe physical phenomena in blue-emitting organic semiconductors including $\pi$-conjugated polyfluorene and related compounds. The work emphasizes molecular structure and intermolecular self-organization that typically determine transport and optical emission in $\pi$-conjugated oligomers and polymers. In this context, hydrostatic pressure through diamond anvil cells has proven to be an elegant tool to control molecular and intermolecular interactions and structure without chemical intervention. High pressure optical spectroscopy has been highlighted in a variety of $\pi$ - conjugated systems whilst analogous X-ray diffraction experiments remain scarce. By focusing on a class of blue-emitting conjugated polymers, polyfluorenes, this article reviews optical spectroscopic studies under hydrostatic pressure, addressing the impact of molecular and intermolecular interactions on optical excitations, electron-phonon interaction, and changes in backbone conformations. This picture is connected to the optical high pressure studies of other $\pi$-conjugated systems and emerging X-ray scattering experiments from polyfluorenes which provides a structure-property map of pressure-driven intra and interchain interactions. Key obstacles to obtain further advances are identified and experimental ways for their resolution are suggested.
\end{abstract}

Keywords: High pressure, conjugated polymers, polyfluorene (Some figures may appear in colour only in the online journal)

\section{Contents}

1. Introduction

2. $\pi$-Conjugated polymers and molecules

3. Experimental techniques and challenges

3.1. Optical spectroscopy

3.2. X-ray scattering

4. Electronic states under pressure

4.1 Singlet states
4.1.1 Photoluminescence energy: insights into backbone planarity
4.1.2 Photoluminescence line-width: inter-chain versus intra-chain interaction
4.1.3 Photoluminescence in PF2/6: the nexus between phase change and, defect and triplet states

4.2 Triplet and polaron states

5 Vibrational states under pressure
5.1 Insights into backbone planarity in oligophenyls

5.2 Mode Grüneisen parameter

5.3 Raman spectra of PF2/6 and PF8 under pressure 5.3.1 Enhanced electron-phonon interaction in PF2/6

6 Structure under pressure

6.1 Structural analogues

6.1.1 $\pi$-Conjugated molecules

6.1.2 $\pi$-Conjugated polymers

6.1.3 Rigid rod polymers and $\pi$-conjugated rigid rod polymers

6.2 Polyfluorene PF2/6

6.2.1 Theoretical considerations

6.2.2 Pressure induced structural effects

6.2.3 Structural inhomogeneity under pressure

7. Summary and Outlook 


\section{Introduction}

In this review, we describe the status of current high pressure research of organic semiconductors focusing on electronic, optical, and structural aspects of $\pi$-conjugated blue emitting molecules and polymers. Although high pressure science of organic semiconductors has been a less studied subfield of semiconductor physics, high pressure research, and polymer science, it has received a renewed interest with advances in new instrumentation, materials synthesis and theoretical methods.

High pressure materials physics is largely based on the pioneering work of P. W. Bridgman (Nobel Prize in Physics1946), who developed important techniques and made a variety of measurements on the macroscopic properties of condensed matter systems [1-3]. Over the years, Bridgman's pressure cell underwent several modifications including anvil devices for ultra-high pressures by Drickamer and co-workers [4, 5]. Afterwards Merrill-Bassett type diamond anvil cells (DACs) opened the most rich avenues for studying phonons and related phase transitions using high pressure Raman spectroscopy and other optical spectroscopic techniques. The DACs also allowed sensitive determination of the crystal structures using X-rays [6].

In the area of inorganic semiconductors, the DACs have played a major role in unravelling the electronic structure of materials. Mail stones include detailed band structure of tetrahedral semiconductors as realized in the early 1960s by William Paul [7]. Independent of the class of inorganic semiconductors - Group IV (Si, Ge, and Sn) or Group III-V (GaP, GaAs, InSb, and AlSb) - the work reported by Paul showed that the pressure coefficient of the direct energy gap (in $k$ space) at the $\Gamma$ point falls in the range of 100-150 $\mathrm{meV} / \mathrm{GPa}$, that of the indirect gap at the $L$ point as $\sim 50$ $\mathrm{meV} / \mathrm{GPa}$, and that of the indirect gap at the $X$ point between -10 to $-20 \mathrm{meV} / \mathrm{GPa}$. Elsewhere, the dielectric properties of Group IV, III-V and II-VI semiconductors were measured in the late 1950s by Cardona and co-workers [8]. Characteristically, the pressure effects of most semiconductors were found to be dominated by the change in polarizability with volume. Such insights from pressure experiments provided guidance to predict the band structure of new alloy semiconductors at atmospheric pressure.

Equally important has been the role of high pressure in understanding the nature of superconductors including superconductivity for different Si phases $[9,10]$. On the basis of an electron-phonon coupling mechanism, superconductivity was theoretically predicted in the simple hexagonal phase of $\mathrm{Si}$, and the transition temperature $\left(\mathrm{T}_{\mathrm{c}}\right)$ was measured as $8.2 \mathrm{~K}$ at $15 \mathrm{GPa}$. In high $\mathrm{T}_{\mathrm{c}}$ superconductors, $15 \mathrm{GPa}$ pressure is known to enhance $\mathrm{T}_{\mathrm{C}}$ above $150 \mathrm{~K}$ in Hg-1223 compounds [11].

There has also been a huge effort in pushing the limit of normal high pressure techniques in the hundreds of GPa range [12-14] whereas shock waves have allowed an access to an experimental pressure-temperature phase space range beyond $1000 \mathrm{GPa}$ and $10,000 \mathrm{~K}$ [15]. Such a pressure range is relevant to the area of planetary sciences and geophysics to linear dispersion, as described by a Dirac-like effectiv better understand the makeup of the Earth's interior.

Some of the earliest high pressure studies of $\pi$ conjugated polymers concerned polyacetylenes (PAs) [1618]. In these studies, the conductivity of iodine doped PA was found to increase till $\sim 0.4 \mathrm{GPa}$ with pressure, indicating a pressure-induced crossover from the critical regime to the metallic regime, followed by a decrease with a further pressure increase. These papers were followed by numerous high pressure works on $\pi$-conjugated systems mostly concerning optical properties.

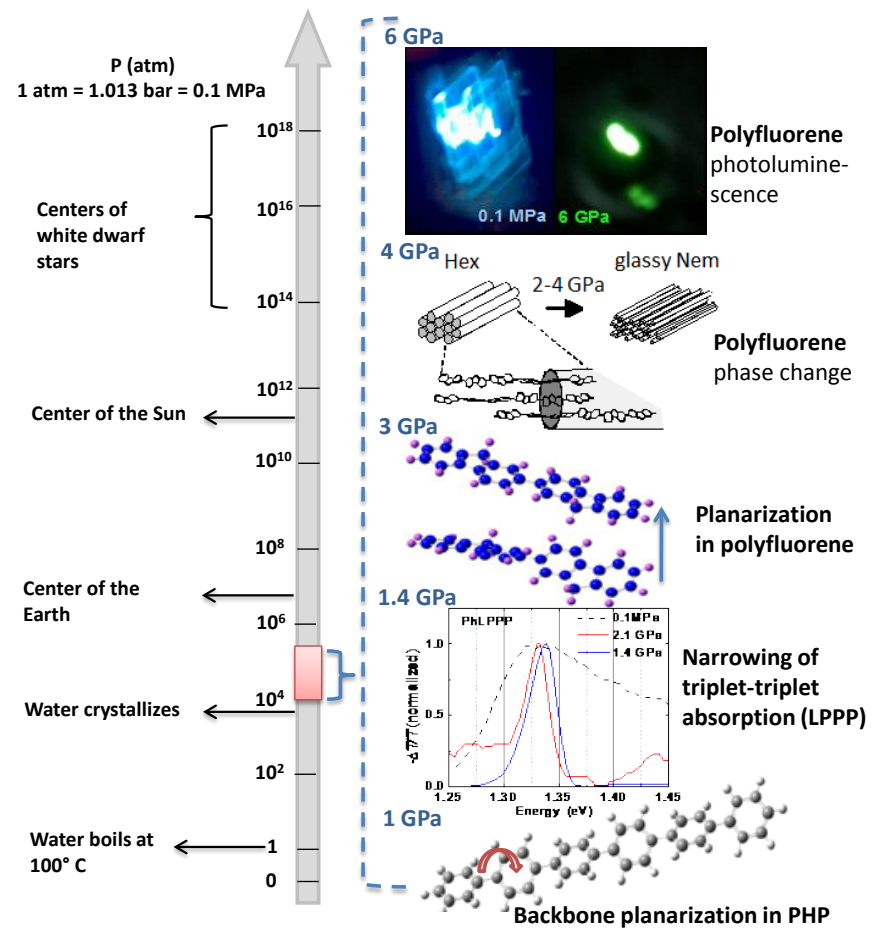

Figure 1. The pressure range relevant to various processes and electronic, optical and structural changes in $\pi$-conjugated molecules and polymers under pressure. The logarithmic vertical scale is in atmospheres. Adapted from Ref. [19].

There are several review papers and monographs published in the area of high pressure techniques and its application to inorganic semiconductors. However, to date there is no comprehensive review in the area of high pressure studies of organic semiconductors $-\pi$-conjugated molecules and polymers - or in the area of optically active soft materials. Our goal here is to bridge this gap by highlighting the effect of high pressure on $\pi$-conjugated molecules and polymers. Much of the physics of these materials can be understood by considering blue-emitting molecules that allow easy emission tuning towards longer wavelengths and utilization in white light-emitting diodes. Moreover, these molecules serve as archetypical rigid rod or hairy rod type molecules which form a basis when understanding their intermolecular self-organization. For these reasons, we build our discussion around blue-emitting organic molecules and illustrate how pressure is used to control their structure, molecular and intermolecular interactions without chemical intervention. 
Figure 1 shows various processes as a function of pressure in our universe along with some key insights obtained from optical spectroscopy relevant to blue-emitting conjugated polymers and molecules. Much of the exciting physical phenomena in the class of blue-emitting molecules and polymers happen at a pressure range from 1-10 GPa. Recent $\mathrm{x}$-ray scattering experiments from $\pi$-conjugated polymers show also relevance of pressure measurements above $10 \mathrm{GPa}$, where structural inhomogeneity starts to play a role.

This review is organized as follows: First, we briefly introduce $\pi$-conjugated molecules and polymers that have been subjected to high pressure research as well as experimental aspects and challenges. This is followed by a detailed discussion of pressure induced effects covering aspects from electronic and vibrational states to intra- and intermolecular structure. It is then illustrated how high pressure research devoted to optical, electrical and structural properties of $\pi$-conjugated polymers may provide new directions for chemical synthesis, and a mechanism for understanding the complex nature of soft materials in general. The summary section casts a glance on some new directions where high pressure may play a role towards understanding soft materials. Throughout this review, particular attention is placed on polyfluorene type materials.

A note on units: we have tried to be consistent in this review and report pressure in the SI unit $(\mathrm{Pa})$. However, many published papers in the past reported pressure in a nonSI unit (bar); figures originating from these works may be labelled in bar and kbar.

\section{2. $\pi$-Conjugated polymers and molecules}

$\pi$-Conjugated molecules and polymers have attracted widespread attention due to their use as low-cost and largearea optoelectronic and photonic devices [20]. Semiconducting properties of these materials are defined by their charge transport along the chain due to the $\pi$ conjugation or between adjacent chains due to the overlapping $\pi$-orbitals between neighboring molecules [21]. In these compounds the carbon atoms have $\mathrm{sp}^{2}+\pi$ bonding and the electronic states mix to form the $\pi-\pi^{*}$ levels, i.e., the highest occupied molecular orbital (HOMO) and the lowest unoccupied molecular orbital (LUMO) energy bands.

The performance of organic devices such as organic light-emitting diodes (OLEDs) [22, 23], thin film transistors [24-26], and solar cells [27, 28] is determined by the chemical structure of individual molecules and the supramolecular organization of the molecule network. Almost all $\pi$-conjugated polymers are made solution processable by flexible side chains which lead to complex molecular microstructure with a critical influence on photonic properties, charge-carrier mobility, morphology, steric hindrance and electronic effects among other things [29-31].

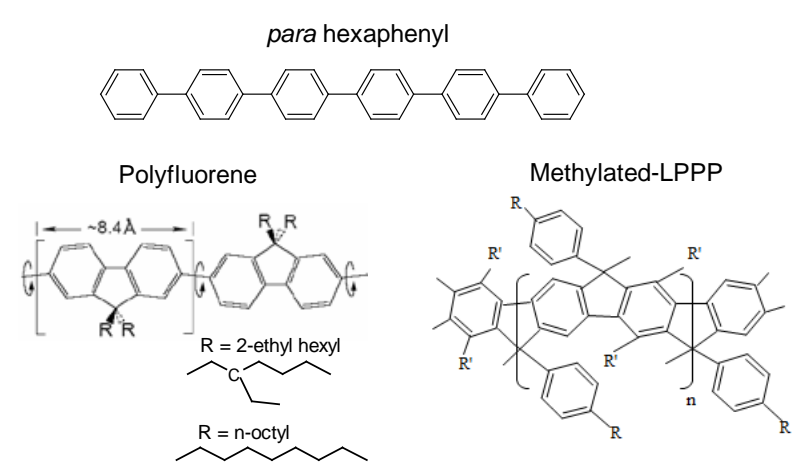

Figure 2. Chemical structure of blue-emitting poly para phenylenetype molecules and polymers. For polyfluorene, $\mathrm{R}$ is an alkyl side chain group. For MeLPPP: R is n-decyl and R' is n-hexyl.

Figure 2 shows chemical structures of four blue-emitting poly para phenylene (PPP) derivatives that has been employed in high pressure studies: Para hexaphenyl (abbreviated as PHP), polyfluorenes (PFs) with 2-ethylhexyl side chains (poly [9,9-bis(2ethylhexyl)fluorene-2,7 diy] (PF2/6) or with n-octyl side chains (poly(9,9-dioctylfluorene) (PFO or PF8), and methylated ladder-type PPP (MeLPPP).

PHP manifests a space group P21/a and a monoclinic unit cell with $\mathrm{a}=8.09 \AA \mathrm{b}=5.56 \AA \mathrm{c}=26.24 \AA$ and $\beta=98.17^{\circ}$ $[32,33]$. In this structure, the single carbon-carbon bond between adjacent phenyl rings introduces a torsion angle (or dihedral angle) $\tau$. The long axis of the unit cell defines the caxis and connects two layers of PHP molecules whereby each layer is represented by two molecules. The plane defined by other crystallographic axes (ab-plane) is parallel to the PHP layers. The nature of the torsional motion between the neighboring phenyl rings is determined at least by two factors: intramolecular repulsion between the ortho hydrogen atoms and the tendency toward planarization in a $\pi$-electron system due to the resonance interaction. Simulations of crystalline packed PHP show a movement of the phenyl rings in a symmetrical potential where the amplitude of the torsions is approximately $\pm 20^{\circ}$ [34].

MeLPPP possesses a permanently planar backbone conformation due to the methyl bridges resulting in low defect concentration [35-38]. In terms of the backbone conformation, PFs lie in between PHP and MeLPPP; within the monomer there is no torsional freedom but adjacent monomers manifest torsional freedom due to the carboncarbon single bond.

Side chain substitutions in polyfluorenes (PFs) confer new functionality and dictate the backbone confirmation. In this review, we will touch upon two PFs: an archetypical branched side chain PF2/6 and an archetypical linear side chain substituted PF8. Besides modifying torsional freedoms, the alkyl side chains in PFs give rise to a rich array of mesomorphic behavior as well as the appearance of the liquid crystalline phase (n-LC) at temperature above $\sim 160^{\circ} \mathrm{C}$.

Figure 3 illustrates ambient structure of PF2/6. PF2/6 adopts a 5- [39, 40] or 21-helical [41, 42] main chain conformation. In the solid state, helical polymers assume bundles of three which self-organizes into a hexagonal (Hex) phase which turns to a nematic (Nem) phase with increasing temperature and decreasing molecular weight. In Ref. [43] 
this transition is understood theoretically in terms of temperature vs. molecular weight binodal as

$$
t \approx A\left(1-\frac{M_{n 0} *}{M_{n} *}\right),
$$

where

$$
M_{n 0} * \approx M_{u} \frac{l_{K}^{2} l_{u}}{v} \ln \frac{e}{f},
$$

is the number averaged molecular weight at which Hex-Nem transition occurs at low temperatures. Here $M_{u}$ and $l_{u}$ are the molecular weight and length of one repeat unit, $l_{K}$ and $v$ are the statistical length and the volume of the segment, $e$ the Euler number and $f$ the volume fraction of the backbone. The value for $M_{n 0}^{*}$ can be calculated in dimensional units which gives $M_{n 0}^{*} \approx 10^{4} \mathrm{~g} / \mathrm{mol}$, corresponding to the experimental data. If $M_{n}<M_{n 0}^{*}$, the polymer is denoted as low molecular weight $\mathrm{PF} 2 / 6$, i.e., LMW-PF2/6. If $M_{n}>M_{n 0}^{*}$, the polymer is denoted as high molecular weight PF2/6, HMW-PF2/6. Parameter $A$ includes experimental parameters and was found to be $\approx 90 \mathrm{~K}$ when fitted to the experimental data. The experimental data suggest that the LMW nematic phase consists of polymer 3-mers and HMW of individual polymers. In the ambient conditions, LMW nematic is the densest form followed by hexagonal and HMW nematic phases.

(a)

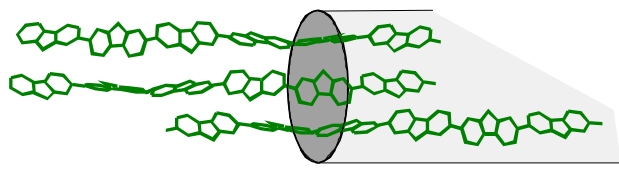

(b)

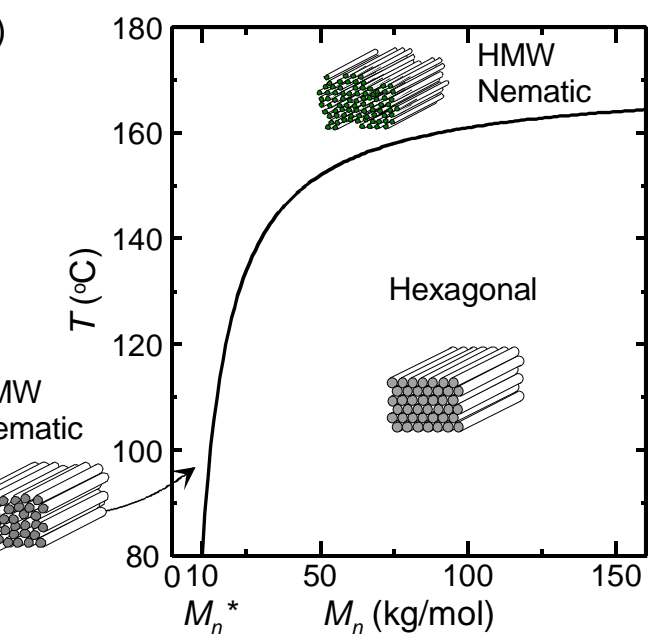

Figure 3 (a) PF2/6 helices (green) forming ordered 3-mers (gray). (b) Phase diagram and intermolecular Hex and Nem structures as a function of molecular weight. $M_{n}^{*}$ is the transition molecular weight separating LMW and HMW materials. Reproduced with permission from [44]. Copyright 2014, Wiley Periodicals, Inc.

The structure of PF8 should be assessed both in terms of its intermolecular structure (also denoted as a phase) as well as its backbone conformation. Bulk PF8 is polymorphic showing crystalline $\alpha$ and $\alpha^{\prime}$ phases and non-crystalline amorphous, glassy, nematic and isotropic phases as well as a mesomorphic $\beta$ phase and a (twisted glassy) g phase that all depend upon the thermal history and the solvent treatment [45-47].

The main chain conformation may be understood in terms of conformational isomers introduced by Chunwaschirasiri et al. [48]. A molecular modeling study shows at least three different "families" for conformational isomers that define the backbone torsional angle, as shown in Figure 4 The most planar conformation, $\mathrm{C}_{\beta}$, is characterized by a torsion angle of $165^{\circ}$ between adjacent monomer units. $\mathrm{C}_{\alpha}$ and $\mathrm{C}_{\gamma}$ refer to more non planar conformations with torsional angles of $135^{\circ}$ and $155^{\circ}$, respectively. These single molecule conformations have their counterparts in the solid state such that the crystalline $\alpha$ is indeed compatible with the $\mathrm{C}_{\alpha}$ conformation and the mesomorphic $\beta$ phase with the $\mathrm{C}_{\beta}$ conformation.

A question that arises: how is the backbone conformation related to the side chain conformation in PF8? After a thermal anneal, the polymer has predominantly $\mathrm{C}_{\alpha}$ and $\mathrm{C}_{\gamma}$ type chromophores. At the simplest level, the relation between the conformational isomers and the side chain conformation can be understood by looking at the gas phase molecular calculations by Chunwaschirasiri et al. [48]. These calculations show that the $\mathrm{C}_{\beta}$ conformer is stabilized when the alkyl side chains adopt an anti-gauche-gauche (agg) conformation. Thus planar backbone units in PF8 require the presence of gauche defects. This is depicted in Figure 4 for a fluorene dimer with two fixed side chain conformations. With an anti-anti-anti (aaa) side chain conformation, the dimer adopts a $\mathrm{C}_{\alpha}$ type non-planar conformation and an agg type side chain conformation leads to a more planar backbone conformation. Although these calculations ignore threedimensional packing they reveal basic attributes to the local intrachain structure that is critically important for high pressure studies.

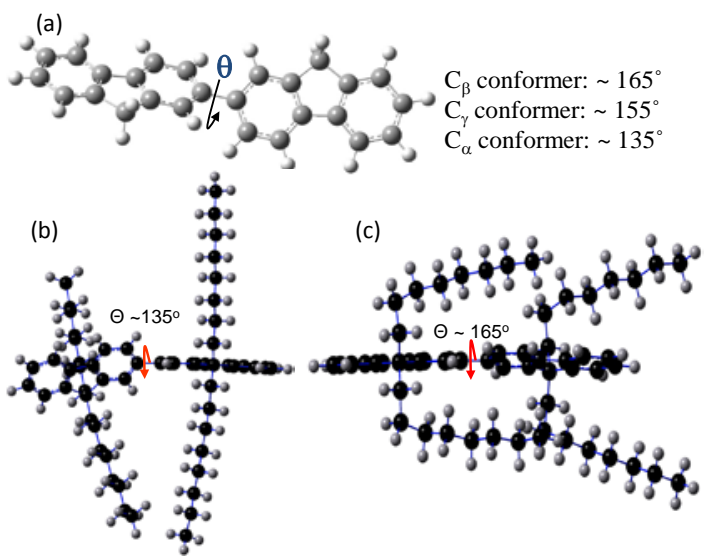

Figure 4. (a) The torsional angle between adjacent monomers in fluorene result in three different conformational isomers. (b) and (c) Fluorene dimer with aaa and agg-type di-octyl side chains. Reproduced with permission from [49]. 


\section{Experimental techniques and challenges} 3.1 Optical spectroscopy

High pressure optical spectroscopy is mainly conducted using a Merrill-Bassett-type DAC. As an example, the ones used at the U. Missouri (MU) consist of two triangular copper-beryllium plates with gem quality diamonds that are cemented to the base as shown in Figure 5 (a). The selection of diamonds for high pressure optical spectroscopy is almost an art by itself. They are designated as type I and II, which refer to the high energy absorption of the stones governed by the impurity content of the carbon lattice. The impurities are typically nitrogen and boron. The diamonds are selected to minimize the luminescence from the stones. For optical absorption measurements, the rare and expensive type II diamonds are preferred due to their smooth absorption spectrum that does not change as drastically as type I with applied uniaxial stress. The cut of the diamond and the alignment of the crystal axis affect the structural integrity of the anvil. The higher the number of facets, the better is the structural integrity; however increasing the number of facets also increases the cost. Typically, culets with 16 facets are used in a DAC and some of them are also beveled, especially in the high pressure range (>100 GPa) [50]

In this typical system, the sample chamber is made out of a stainless steel disc-gasket with a hole drilled in the middle (schematically shown in Figure 5 (b)). The size of the hole is about $200 \mu \mathrm{m}$ while the culet size of the diamonds is about $\sim 1000 \mu \mathrm{m}$. Work hardening by indentation of the gasket is necessary in order to achieve and maintain high pressures in the sample chamber. A thin and a uniformly pressed gasket will allow the cell to easily go up to $\sim 10 \mathrm{GPa}$ without failure. The elastic property of the work-hardened stainless steel allows very high pressure in the sample chamber. Along with the sample, a small piece of ruby is also placed in the sample chamber (Figure 5 (c)). The loading procedure well-suited for organic semiconductors and many modifications to the DAC including designing various jigs for easy loading of the cell at MU were pioneered by Meera Chandrasekhar [51, 52].

For cryogenic loading, it is essential that indium gaskets are used as a seal around the seat of the diamond. Argon used as a pressure transmitting fluid is introduced into the sample chamber via a cylindrical ring attached to a capillary tube as shown in Figure 5 (a). Argon loading is usually done in a liquid $\mathrm{N}_{2}$ bath. After the loading process (where the liquid argon is trapped, bolts are tightened, and the DAC is brought to room temperature), the gasket hole which is the sample chamber usually contracts a bit. The pressure medium inside the sample chamber ensures a uniform hydrostatic environment.

The three bolts on the triangular DAC control the pressure inside the chamber and the ruby fluorescence is a good pressure calibrant. There are two strong ruby fluorescence lines at $694.2 \mathrm{~nm}\left(\mathrm{R}_{1}\right)$ and $692.8 \mathrm{~nm}\left(\mathrm{R}_{2}\right)$ at atmospheric pressure. Shown in Figure 5 (d) are the ruby fluorescence peaks in wavenumber $\left(\mathrm{cm}^{-1}\right)$ for a few selected pressures. Till $20 \mathrm{GPa}$, the fluorescence peaks shift linearly at $-7.53 \mathrm{~cm}^{-1} / \mathrm{GPa}$ [53]. Although at higher pressures the shift of the $\mathrm{R}_{1}$ line becomes nonlinear, Mao et al. have shown that a nonlinear equation yields a reasonable pressure calibration till about 100 GPa [54]. The relative intensity of the two $\mathrm{R}$ lines is sensitive to the temperature, and the line width depends on the hydrostatic environment.

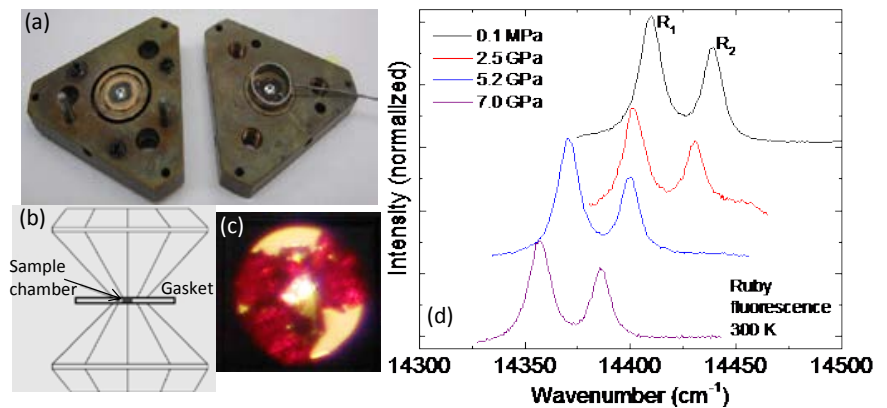

Figure 5. (a) Photograph of a DAC. (b) Schematic of the DAC showing the sample chamber and the gasket. (c) Photograph of a polymer sample (reddish) and a piece of ruby (clear piece) inside the sample chamber of a closed DAC. (d) Ruby fluorescence in wavenumbers at selected values of pressure.

One inherent problem for measuring Raman scattering of conjugated polymers and molecules with a DAC is the strong Raman peak of the diamond itself (at $1330 \mathrm{~cm}^{-1}$ ). Almost all $\pi$-conjugated systems have carbon-carbon backbone stretch frequencies in the $1300 \mathrm{~cm}^{-1}$ range in the Raman spectra, which cannot be tracked with pressure as the diamond peak overwhelms this region. Sapphire anvils, which have almost no Raman peaks above $1000 \mathrm{~cm}^{-1}$, are an option [55], although they are more prone to being damaged during the alignment process.

\subsection{X-ray scattering}

High pressure X-ray diffraction (XRD) and scattering methods in the GPa pressure range are well-established for inorganic materials [56] and organic solids [57] and used to reveal complicated features of water [58], for example. The literature for polymers is far less comprehensive. The main emphasis has been in the MPa range where XRD has been employed to construct full phase diagrams as functions of temperature and pressure as shown e.g. for melt poly(4methyl-1-pentane) [59]. When GPa range studies are concerned, the focus has been on relatively simple model polymers including polyethylene [60] and poly(tetrafluoroethylene) (PTFE) [61-63]. These studies have revealed compression of an ambient phase as well as several new high pressure phases.

Why the main stream of polymer science has been off the extreme condition research stems mainly from experimental challenges. In high pressure XRD, we should ideally obtain unit cell parameters as a function of pressure and temperature. This would allow understanding not only about materials structure but also about pressure dependent packing density. Polymers are typically semi-crystalline which means that the material contains both crystalline and amorphous domains. They are multiphase systems such that several crystalline and non-crystalline phases can coexist and the fraction of all phases can be significant. This means that we should determine pressure dependent change not only in 
their crystalline structure but also in their domain fractions. Similarly, we should follow packing density not only for individual phases but also for their overall mixture. This kind of overall analysis has turned to be an overwhelming task.

Generating high pressure requires small samples. Small samples mean weak signal per se and thus careful control of the scattering background. This is challenging to polymers which display small crystallites and magnitudes lower structural order than inorganic materials. Small crystallite size also means that the peaks are broad and appear as bundles of peaks, making analysis tricky. This requires several experimental measures including, among other things, careful beam collimation and the use of perforated diamonds [64].

To understand polymers we should consider intra- and intermolecular order on multiple length scales from Angstroms to the submicron scale. Similarly, the phase transitions at different length scales are associated with different energy levels and thus different pressure levels. This means that the scattering should be detected over wide range of scattering angles and the pressure screened both in the $\mathrm{MPa}$ and GPa range. Instruments allowing such a broad spectrum of measurements are rare.

Polymers suffer from radiation damage. This becomes particularly complicated with small culet size, which requires small and intense X-ray beam, and when the required thermodynamical characterization prevents the use of cryostats. Other challenges of polymer research under high pressure are that polymers may interact, become dissolved or form co-crystals with alcohols or other pressure transmitting media.

\section{Electronic states under pressure}

There are many excellent accounts of optical processes in organic materials [65-68]. The brief overview in this section is intended to provide a background for understanding the optical spectra of luminescent conjugated materials under high pressure. The photophysics of conjugated organic materials exhibits several excitation and relaxation processes, as depicted in Figure 6. The basic absorption and photoluminescence (PL) processes are observed as electronic transitions between the singlet ground and excited states. Upon absorption of a photon, a neutral excited state- the singlet exciton - is created followed by both radiative (PL) and non-radiative recombination processes. In molecular materials, the singlet excitons are Frenkel-type, which are tightly bound with large binding energies $(\sim 1 \mathrm{eV})$. Through a change in their spin multiplicity singlet excitons may populate the triplet exciton states, which have long lifetimes. Triplet generation can be probed by triplet-triplet $\left(\mathrm{T}_{1}-\mathrm{T}_{\mathrm{N}}\right)$ absorption using modulation spectroscopy. The singlet excitons may further dissociate to form singly charged polarons or polaron pairs resulting in a polaronic absorption. The polarons are generated either via exciton dissociation (electric fields, defects, and electron or hole acceptors) or via direct creation (charge injection or doping mechanisms). In addition to the spin $1 / 2$ polarons, spinless bipolarons occur, which arise from gap states that are deeper than the polaron states. The population of the polaron and triplet states leads to a small depopulation of the ground state, giving rise to a bleaching of the absorption.

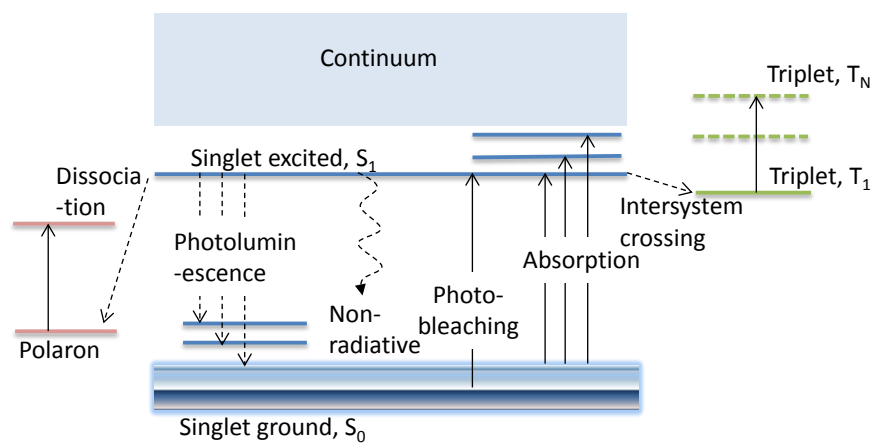

Figure 6. A schematic diagram of energy levels in conjugated molecules and polymers. The singlet states are involved in the process of absorption and photoluminescence, including photobleaching. The triplet state, $T_{1}$, is populated from the $S_{1}$ via intersystem crossing. The polaronic states may results from a dissociation of the singlet excitons.

The absorption and PL emission spectra of conjugated polymers and molecules are characterized by a well-resolved Franck-Condon (FC) type progression of the vibronic subbands in addition to the main $\pi-\pi^{*}$ electronic transition. In the adiabatic picture, a vibronic progression in the electronic spectra implies that the ground and excited state equilibrium structures are displaced relative to one another in configuration space, as shown in Figure 7. The absorption and the PL spectra typically show a Stokes shift, which is related to the displacement of the ground and excited states $(\Delta)$. If there is no displacement of the potential energy curve in the excited state relative to the ground state, only a single absorption line would be allowed due to the orthogonality of the vibrational wavefunctions. Since excited-state distortions mainly arise from bond alternation, the coupling is the strongest for the carbon-carbon bond stretching modes in the $1200-1600 \mathrm{~cm}^{-1}$ region. Additional vibronic replicas, representing the coupling of weaker phonon modes to the electronic transitions, are also observed between the main vibronic peaks.

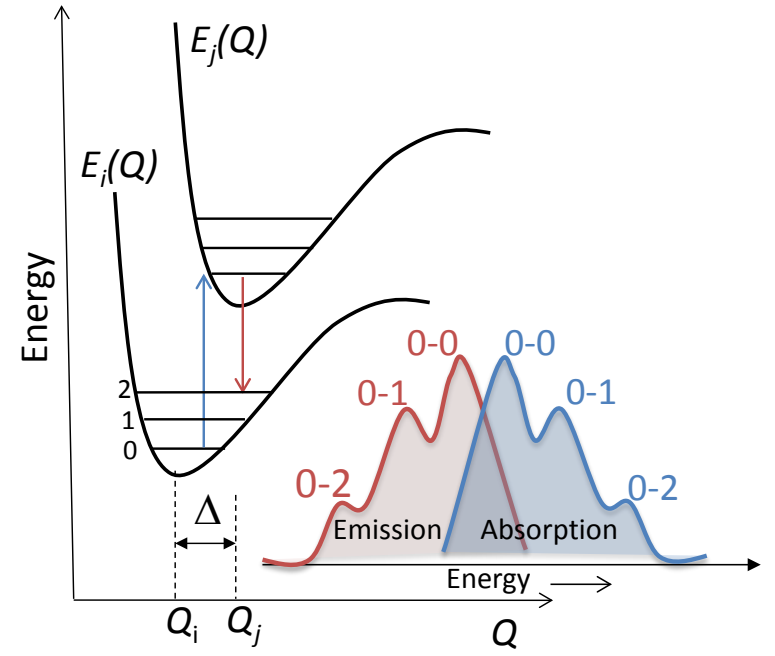

Figure 7. The electronic ground and excited states in configuration 
space. The inset shows a schematic of the absorption and PL spectra.

It is easy to understand the mirror-image rule between absorption and PL spectra as schematically shown in the inset of Figure 7. PL occurs from the lowest vibrational state; the emissive transition highest in energy is the $0-0$ transition, which takes place between zeroth vibronic level in the excited state and the zeroth vibronic level in the ground state. At the simplest level, one may think of a long-chain conjugated polymer chain being composed of a series of 1-D potential wells with varying well widths, reflecting conjugation breaks. Absorption occurs at all sites as long as the incident energy is higher than the $\pi-\pi^{*}$ gap of all sites. Exciton formation occurs usually in sub-picosecond time scales followed by excitation migration to the longest chain segment (with the lowest energy), which occurs in tens of picoseconds [69]. The PL occurs from the lowest energy site. In essence, absorption samples all conjugated segments whereas PL represents the longest chain segment in a conjugated polymer.

In the emission process, the Huang-Rhys factor $(S)$ corresponds to an average number of phonons that are involved when an excited molecule relaxes to its ground state configuration from its new equilibrium configuration in the excited state [67]. Assuming that the vibrational frequency is the same for ground and excited states and that the potentials are perfectly parabolic, $S$ may be experimentally determined from the fractional intensity of the vibronic peaks. The relative intensities of the features coupled by a single phonon frequency $(\omega)$ are described by

$$
\frac{I_{0 \rightarrow n}}{I_{\text {total }}}=\frac{e^{-S} S^{n}}{n !}
$$

where $I_{\text {total }}$ is the total intensity of the individual transitions. $I_{0 \rightarrow n}$ is the intensity of the transition from the $0^{\text {th }}$ vibronic excited state to the $n^{\text {th }}$ vibronic state of the electronic ground state. The Huang-Rhys factor therefore corresponds to the average number of phonons that are involved when the excited molecule relaxes to its ground-state configuration from the excited state with $S \hbar \omega$ as the relaxation energy.

Using a configuration co-ordinate model, Drickamer and co-workers came up with a scheme for obtaining the change in volume of the system upon electronic excitation and the ratio of the force constants of the excited and the ground states from high pressure optical data [70-72]. We do not describe this model in detail here but it becomes particularly useful when structural data of systems under pressure are not available. In pressure-induced thermal and optical processes, pressure selects volume of the system as the appropriate configuration coordinate. If one assumes a single configuration coordinate, as shown in Figure $7, \Delta$ can be obtained from the dependence of the PL energy and linewidth as a function of pressure. Additionally, if details of the absorption spectrum as a function of pressure are available, an independent verification of $\Delta$ may be achieved. The effect of pressure on the electronic excitation is strongly coupled to the intermolecular displacement, resulting in a decrease in configuration coordinate (volume) upon excitation, which is further related to an enhanced attractive force between the molecule and the surroundings.

\subsection{Singlet states}

An understanding of the influence of intermolecular interactions is crucial since the photoluminescence quantum yield (PLQY) of conjugated polymers is known to decrease in the solid state compared to solutions [73, 74]. Luminescent conjugated molecules and polymers provide an easy access to study the electronic states, and thus the mechanisms that influence PLQY using a DAC.

The absorption and PL spectra of poly ( $p$-phenylene vinylene) (PPV)-type and thiophene-based conducting polymers under pressure were measured in the late 1980s and 1990s [75-78]. Most of these works utilized DACs with either oil or methanol-ethanol mixture as the pressure transmitting fluid. Since PPVs have a torsional degree of freedom in the backbone, the absorption spectrum does not show any vibronic structure. Moreover, the polydispersity of conjugated polymers also play a role in smearing out the vibronics in the optical spectra due to a range of conjugation lengths.

Figure 8 shows the PL spectra of MeLPPP film, PF2/6 film, and bulk PHP. In all three cases the vibronic progression is clearly seen at $0.1 \mathrm{MPa}$, smearing out at high pressures. The $0-0$ transition in PF2/6 is observed at $\sim 2.95$ $\mathrm{eV}$, followed by the $0-1$ transition at $2.78 \mathrm{eV}$. The MeLPPP film shows the $0-0$ transition at $2.68 \mathrm{eV}$, followed by the $0-1$ peak at $2.52 \mathrm{eV}$. The PHP sample shown in Figure 8 is from bulk powder; the 0-0 transition is thus not observed due to self-absorption at $0.1 \mathrm{MPa}$. Thin evaporated films show the $0-0$ transition at $3.12 \mathrm{eV}$ followed by the $0-1$ transition at $2.95 \mathrm{eV}$ [79]. Since the overall PL spectrum red-shifts under pressure, the 0-0 transition in PHP is observed at higher pressures as shown in Figure 8.

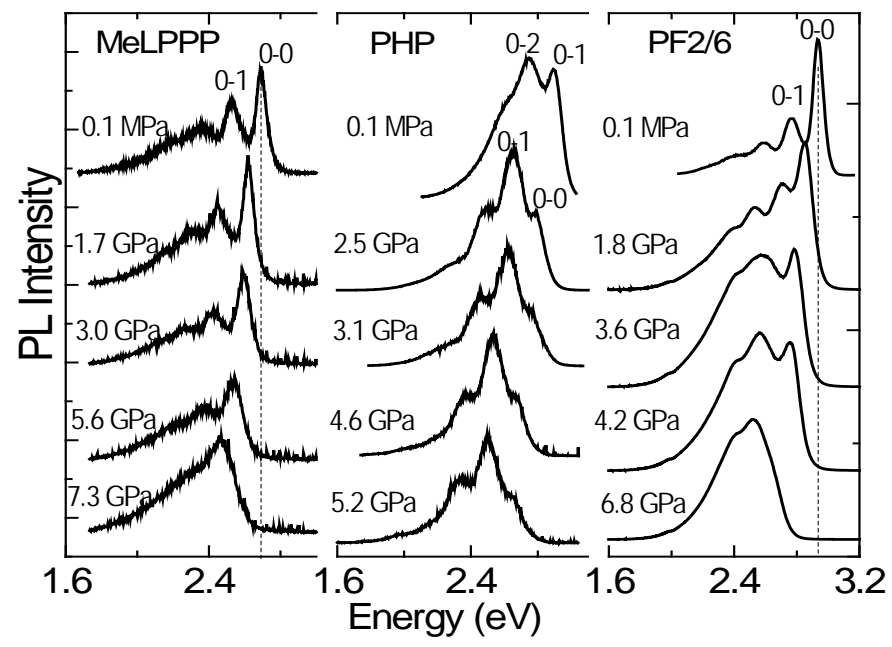

Figure 8. PL spectra of MeLPPP film, PHP, and PF2/6 film at selected values of pressure at $300 \mathrm{~K}$. Reproduced with permission from [80]. Copyright 2004 Wiley Periodicals, Inc.

Since MeLPPP has a planar backbone without any torsional degree of freedom, the absorption spectrum shows a vibronic progression even under pressure, as shown in Figure 
9. The absorption edge typically red-shifts at the same rate as the PL energies. The broadening of the individual vibronics is seen in most amorphous conjugated polymers, and is discussed in detail in Section 4.1.2.

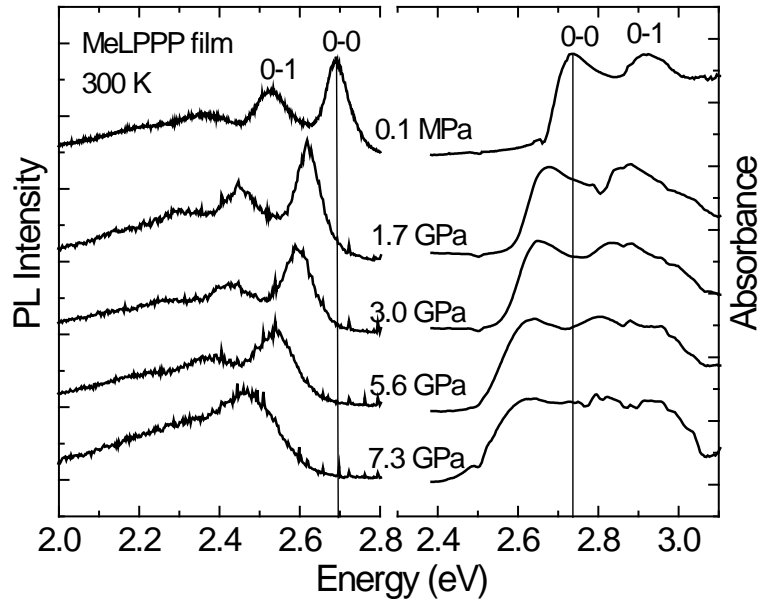

Figure 9. PL and absorption spectra from MeLPPP film at selected values of pressure at $300 \mathrm{~K}$. Reproduced with permission from [81]. Copyright 1999 Wiley Periodicals, Inc

\subsubsection{Photoluminescence energy: insights into backbone planarity}

In this section we look into the details of the red-shift in PL/absorption energies and how it relates to specific backbone conformations. As shown above, all $\pi$-conjugated polymers and molecules exhibit a red-shift in PL and absorption energies under pressure. This is in contrast to inorganic semiconductors such as GaAs and GaN, where the band to band as well as defect (shallow and deep) states are seen to show a net blue-shift in their energies with increasing pressure [82-84]. As an example of an inorganic semiconductor, the direct gap transition $\left(E^{\mathrm{I}}\right)$ of $\mathrm{GaAs}$ in a $(20,20) \mathrm{GaAs} / \mathrm{AlAs}$ superlattice is shown in Figure 10. Between $0.2 \mathrm{GPa}$ and $0.7 \mathrm{GPa}$, the $\mathrm{E}^{\mathrm{I}}$ transition blue-shifts by more than $60 \mathrm{meV}$. The linear pressure coefficient of the $\mathrm{E}^{\mathrm{I}}$ transition in this superlattice is similar to bulk GaAs $(\sim 100$ $\mathrm{meV} / \mathrm{GPa}$ ) [84]. In such direct band gap semiconductors, the p-type valence band and the s-type conduction band as schematically shown in Figure 10 (b) may shift at different rates, resulting in a net blue-shift in the optical band gap.
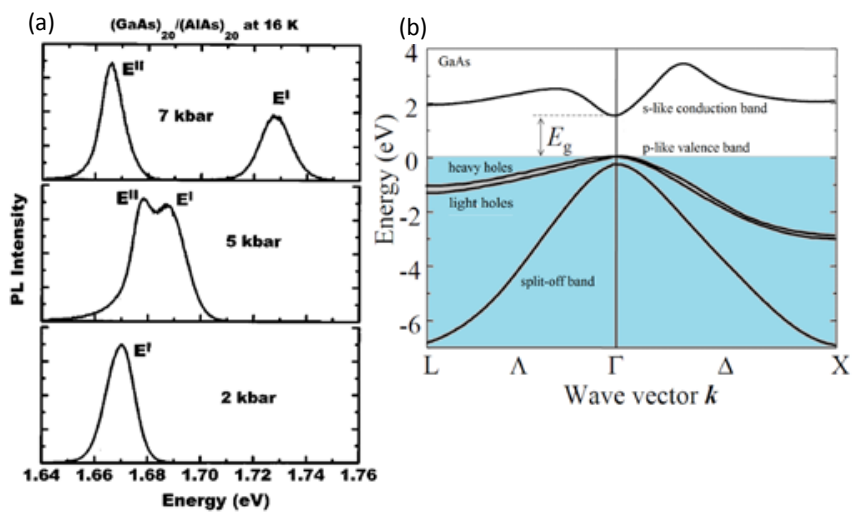

Figure 10. (a) PL spectra of a GaAs/AlAs $(20,20)$ superlattice, showing the blue-shift of the direct gap transition in GaAs $\left(\mathrm{E}^{\mathrm{I}}\right)$ with increasing pressure. Reproduced with permission from [84]. Copyright 1998 The American Physical Society. (b) Schematic of the band structure of GaAs with its s-like conduction band and p-like valence band. Reproduced with permission from [85]. Copyright 1976 The American Physical Society.

The red-shift of electronic energies under pressure has been attributed to a "gas-to-crystal" shift, which arises from dipole-dipole interaction, lowering the excited state energy relative to the ground state energy [76]. Essentially, pressure enhances the conformational ordering, resulting in a higher degree of the overlap of the $\pi$-electron wavefunctions. Stateof-the-art $a b$ initio calculations from high pressure studies of oligo para-phenylenes show that the red-shift in the absorption spectrum is mainly intermolecular in origin [86]. A reduction in the inter-ring bond lengths enhances the $\pi$ conjugation of the molecules [87]. Further advancement of theoretical methods, which explicitly consider the electronhole pairs within a Bethe-Salpeter formalism in oligoacenes, predict an accurate trend in the red-shift of the absorption edge in anthracene under pressure along with an estimation of the exciton binding energy [88].

Although $\pi$-conjugated polymers and molecules show a red-shift in their PL energies, their pressure coefficients (both linear and non-linear) can vastly differ depending on their backbone conformation. Figure 11 (a) shows the 0-0 and the 0-1 PL vibronic energies for PHP, PF2/6 and MeLPPP at 300 $\mathrm{K}$. Below $1 \mathrm{GPa}$, it is often difficult to tune the pressure at room temperature; lowering the temperature provides a better tunability of pressure in a DAC. PHP shows a sharp change below $1 \mathrm{GPa}$, as indicated by the dotted rectangle in the figure. All the vibronic peaks are clearly seen at $16 \mathrm{~K}$ (between $0.1 \mathrm{MPa}$ and $1 \mathrm{GPa}$ ), and their energies are plotted in Figure 11 (b). A sharp change in the energies is found at low pressures for PHP. PF2/6 also shows a slight deviation from a linear shift below $2 \mathrm{GPa}$. These sharp changes in PL energies reflect a partial planarization of the backbone, i.e., a reduction in the torsional angle between the neighboring phenyl rings or adjacent units.
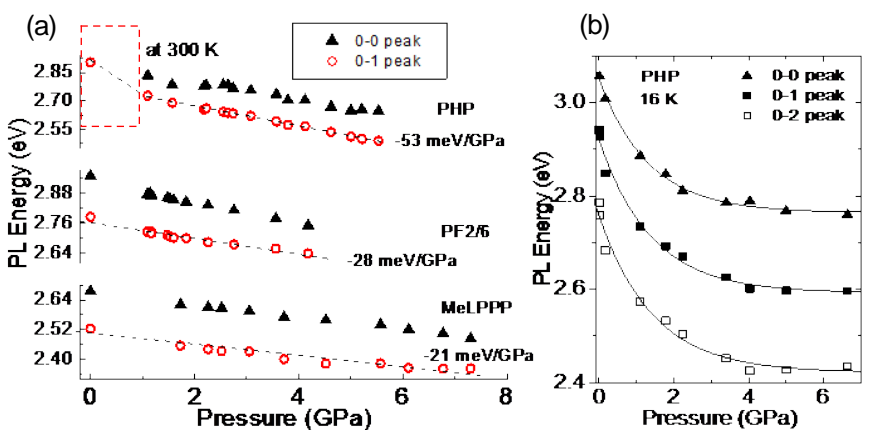

Figure 11 (a) PL peak positions of the $0-0$ and $0-1$ transitions in PHP powder, PF2/6 film, and MeLPPP film as a function of pressure at $300 \mathrm{~K}$. (b) PL peak positions of a PHP powder sample as a function of pressure at $16 \mathrm{~K}$. Adapted from Refs. [80, 89].

Table I summarizes the linear pressure coefficients of the vibronic peaks of various conjugated polymers/molecules. The energy shift is described by: $E(P)=E(0)+\alpha P$, where 
$E(P)$ is the energy at pressure, $P, E(0)$ is the energy at ambient pressure, and $\alpha$ is the pressure coefficient. The PHP data at $16 \mathrm{~K}$ is fit only till $2 \mathrm{GPa}$. The two highlighted rows in the table depict systems with high pressure coefficients. These systems further show a partial planarization of the backbone under pressure. Raman scattering and IR (infrared) absorption are excellent probes to detect planarization of the backbone in oligophenyls, as discussed in Section 5.1

Table 1 Pressure coefficients of $0-0$ and $0-1$ PL vibronic peaks. Only the linear pressure coefficients are shown. Ref. [89].

\begin{tabular}{lcc}
\hline Sample & $\begin{array}{c}\alpha_{0-0} \\
(\mathrm{meV} / \mathrm{GPa})\end{array}$ & $\begin{array}{c}\alpha_{0-1} \\
(\mathrm{meV} / \mathrm{GPa})\end{array}$ \\
\hline MeLPPP film & -25 & -21 \\
PHP $(16 \mathrm{~K})^{\mathrm{a}}$ & -116 & -135 \\
PHP (300 K) & -44 & -53 \\
PF2/6 film & -40 & -28 \\
PF8 bulk $\left(\mathrm{C}_{\beta}\right)$ & -38 & -37 \\
PF8 bulk $\left(\mathrm{C}_{\alpha} / \mathrm{C}_{\gamma}\right)$ & -51 & -76 \\
PPV $^{\mathrm{b}}$ & -37 & \\
\hline
\end{tabular}

${ }^{\mathrm{a}}$ linear fit till 2.0 GPa; ${ }^{\mathrm{b}}$ Ref. [77].

As described earlier, PF8 shows polymorphism. The overall $\alpha$ crystalline phase consists of individual chromophores which are more non planar ( $\alpha$ or $\gamma$-type). This phase may be induced by thermal cycling the polymer to its n-LC phase. The pressure coefficient of the $\mathrm{C}_{\alpha} / \mathrm{C}_{\gamma}$ PF8 in Table I corresponds to a sample that was thermally cycled in a DAC.

Figure 12 (a) shows the PL spectra of as-is $\left(\mathrm{C}_{\beta}\right)$ and thermally-cycled $\left(\mathrm{C}_{\alpha} / \mathrm{C}_{\gamma}\right)$ PF8 at $0.1 \mathrm{MPa}$. Since the $\alpha$ phase is incompatible with $\mathrm{C}_{\beta}$ conformers, the emission blue-shifts upon thermal cycling. The $0-1$ peak position is plotted in Figure 12 (b) for the as-is and the thermally cycled (TC) sample as a function of pressure. Shown by the shaded region, the PL vibronics from the two samples do not shift in a parallel fashion. Since the TC sample is mainly in the $\mathrm{C}_{\alpha}$ and $\mathrm{C}_{\gamma}$ conformation, pressure has two effects: an enhanced conformational ordering similar to the as-is sample, and planarization of the backbone, driving it towards the planar $\mathrm{C}_{\beta}$ conformation. The nature of planarization in PF8 conformers can be further understood by a simple theoretical model, shown as an inset in Figure 12 (b). Details of the theoretical calculations are provided in Ref. [90].

The calculated energy gap in the inset is the singleparticle HOMO-LUMO gap for flourene dimers using density functional theory (DFT). We point out that the HOMOLUMO gap is not the experimentally observed optical gap. The optical gap arises from many-body excitonic levels, and thus has a lower energy compared to the HOMO-LUMO gap. The $\mathrm{C}_{\beta}$ conformation was modeled as being fully planar with a torsion angle of $180^{\circ}$. The torsion angle for each of the conformers was held constant for the various pressure points. Pressure effects were simulated by compressing the C-C bond between the two monomers in a single chain. The pressure was estimated using the Murnaghan Equation of State, which expresses the unit cell volume $(V(P))$ as a function of P by [91]

$$
V(P)=V_{0}\left(1+\frac{B_{0}^{\prime} P}{B_{0}}\right)^{-1 / B_{0}^{\prime}},
$$

where $\mathrm{B}_{0}$ is the bulk modulus, $B_{0}^{\prime}$ is its derivative, and $V_{0}$ is the unit cell volume at $\mathrm{P}=0$ (optimized geometry). Since the monomer units are connected by $\mathrm{C}-\mathrm{C}$ single bonds, the known bulk modulus of PHP was used in these calculations [90].

The theoretical energies show a non-linear shift with increasing pressure. Experimentally, the TC sample does not simply follow the theoretical phase space of the $\mathrm{C}_{\alpha}$ or $\mathrm{C}_{\gamma}$ conformation as a function of pressure, but rather, due to a planarization of the torsion angle, it may follow an intermediate path, shown by the dash-dot line in the inset. (a)
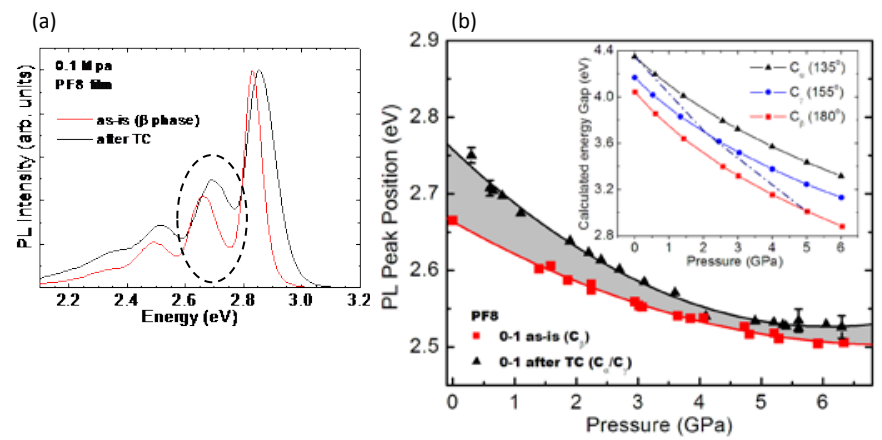

Figure 12 (a) PL spectra of an as-is and thermally cycled PF8 film at 0.1 MPa. (b) The 0-1 PL energy peak position of as-is and thermally cycled PF8 as a function of pressure. The inset shows the calculated energy gap of fluorene dimers with $\mathrm{C}_{\alpha}, \mathrm{C}_{\gamma}$, and $\mathrm{C}_{\beta}$ conformations as a function of pressure. The dashed-dot line is a schematic of a plausible transition for the thermally cycled PF8 sample. Reproduced with permission from [90]. Copyright 2010 The American Chemical Society.

We point out that the compressibility of rigid molecules can be anisotropic. In oligophenyls, a pressure dependent compressibility arises from the two perpendicular axes to the chain axis [33]. For polycrystalline fluorene, it is the hardest to compress the unit cell along the long unit cell direction, which is parallel to the long axis of the fluorene molecule [92]. Non-linear pressure coefficients of the PL and absorption energies in conjugated molecules and polymers thus often indicate a planarization of the backbone. Thus, a red-shift in electronic energies with pressure represents an increased conformational ordering, followed by a planarization of the backbone, especially in systems which possess a torsional degree of freedom. PF2/6, which has a helical backbone, also shows a partial planarization of the backbone. This is now inferred from x-ray scattering data under pressure; details are provided in Section 6.2.

\subsubsection{Photoluminescence line-width: inter-chain versus intra- chain interaction}

PL broadening as a function of pressure sheds light on the nature of intermolecular interactions. In this respect, the amorphous polymers show differences compared to more crystalline systems. The increase in line widths of the PL vibronics is symptomatic of an enhanced inter-chain interaction, observed in most amorphous conjugated polymers. 
Figure 13 shows the full width half maxima (FWHM) of the $0-1 \mathrm{PL}$ peaks for various polymer samples. We note that the actual widths vary from sample to sample; MeLPPP, which has a planar backbone conformation without any torsional degree of freedom, shows the lowest FWHM for the individual vibronics at $0.1 \mathrm{MPa}$. Figure 13 (a) plots the linewidths of PF samples. Annealed (thermally cycled) PF8 is semi-crystalline with the $\alpha$ and $\alpha$ ' phase and does not show a change in its PL linewidth with increasing pressure. The as-is PF8 sample, which is more amorphous, however, shows a monotonic increase in its linewidth with increasing pressure. Plotted in the same figure are also the pressure dependence from two PF2/6 samples, one with a high molecular weight and the other with a low molecular weight (with $\mathrm{M}_{\mathrm{n}}<10$ ). For the HMW PF2/6 sample, the PL linewidth change is quite gradual beyond $2 \mathrm{GPa}$, which is attributed to a weakly ordered state with a partial planarization of the backbone. Detailed XRD measurements from HMW and LMW PF2/6, presented in Section 6.2, shows the similarities and dissimilarities in their structures. LMW PF2/6, which maintains its nematic liquid phase with increasing pressure, shows an increasing PL linewidth with pressure. A similar trend is seen for MeLPPP (Figure 13 (b)). PHP, which is more crystalline, shows an unchanged PL linewidth with pressure.

A 3D band structure DFT calculation of the imaginary part of the dielectric function, $\operatorname{Im}(\varepsilon)$, offers some insights in understanding the origin of line broadening. These calculations were performed for PPP-type molecules. A redshift of the bandgap with almost no broadening of $\operatorname{Im}(\varepsilon)$ occurs when the inter-ring bond between the phenyl rings is shortened in PPP molecules. In contrast, when the inter-chain distance is decreased, $\operatorname{Im}(\varepsilon)$ broadens significantly due to an increased bandwidth of the localized bands perpendicular to the molecule axis [87]. In light of these theoretical results, one can then attribute the PL broadening to an enhanced inter-chain interaction. Polymers such as MeLPPP, which have bulky side chains, show an enhanced inter-chain interaction in this pressure range. In contrast, the herringbone arrangement of molecules in PHP prevents the chains from interacting under pressure; the primary effect in such molecules is a compression of the molecular axis. (a)
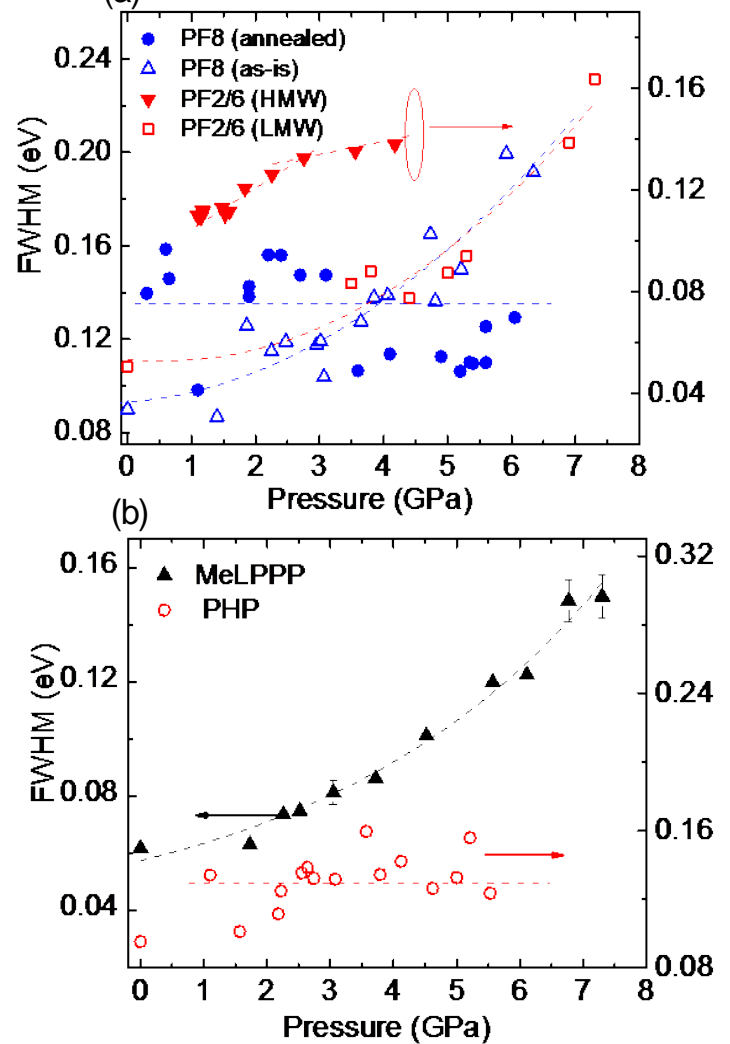

Figure 13 (a) Pressure dependence of the FWHM of the $0-1$ PL linewidths of various PF8 and PF2/6 samples. (b) FWHM of the $0-1$ PL vibronics of MeLPPP films and bulk PHP at $30 \mathrm{~K}$ as a function of pressure. The dotted lines are a guide to the eye and represent the trends. Adapted from Ref. [89].

Time-dependent PL experiments also shed light into the nature of intra-chain versus inter-chain interactions in conjugated polymers/oligomers under pressure. Schmidtke et al. compared a thin film and isolated chains (within an inert matrix) of a copolymer of PF: poly(9,9-di-n-octylfluorenealt-benzothiadiazole) (F8BT) under pressure [93-95]. At higher pressures, the thin film sample showed biexponential PL decay with time constants 0.6 ns and 2.3 ns compared to a monoexponential PL decay rate of 2 ns at $0.1 \mathrm{MPa}$. The greater change in the PL decay rate for the thin film sample compared to the isolated chain sample was attributed to a higher inter-chain interaction, which allows for additional pathways for nonradiative decay channels under pressure.

As briefly outlined in Section 4, Drickamer and coworkers came up with a simple method for obtaining the change in volume of the system upon application of high pressure from the optical data (PL/absorption linewidth and shifts). Although the model is an oversimplification, in that, one assumes only a single configuration coordinate for complex systems, yet it is useful to see the trends of volume change under pressure, especially when complete XRD data under pressure is non-existent. We list the value of $\Delta$ (shown in Figure 7) for the blue-emitting systems discussed in this review and for some other small molecules in Table 2. The negative value suggests a decrease in volume with pressure. One clearly sees that for crystalline systems such as acenes and PHP, the magnitude of $\Delta$ is higher. Thus the impact of 
pressure on the electronic excitations is strongly coupled to the intermolecular displacement, which decreases the configuration parameter with pressure, and this is further related to the attractive forces between the molecule and the surroundings. We direct the readers to Ref. [89] for a detailed discussion on how the configuration coordinate parameter is obtained from the experimental data under pressure.

Table 2 Configuration coordinate parameter. Ref. [89].

\begin{tabular}{cc}
\hline Sample & $\Delta\left(\mathrm{cm}^{3} / \mathrm{mol}\right)$ \\
\hline MeLPPP film & -1.6 \\
PHP & -5.1 \\
PF2/6 film & -2.4 \\
Anthracene crystal* & -10.2 \\
Tertacene crystal* & -11.7 \\
Phenanthrene crystal* & -3.7 \\
\hline
\end{tabular}

*Ref. [72]

\subsubsection{Photoluminescence in PF2/6: the nexus between phase change and, defect and triplet states}

The optical properties of PF2/6, especially HMW samples under pressure, show some striking features not observed in other polymers. These changes may be attributed to a delicate balance between a phase transition and other types of emission resulting from defect and triplet states. Since the changes inferred from the optical data under pressure have now been confirmed by recent XRD experiments, discussed in Section 6, in this section we attempt to elaborate how optical spectroscopy yields insights into phase change, especially in helical polymers.

OLEDs fabricated from the PF family of materials often see degradation in its emission, where the blue electroluminescence changes to a 2.1-2.4 eV greenish/ reddish emission $[96,97]$. This red-shift in the emission has been attributed to keto defect sites (9-fluorenone) as schematically shown in Figure 14 (inset), where the bridging carbon atom forms a $\mathrm{C}=\mathrm{O}$ bond. These defect sites act as guest emitters, efficiently trapping singlet excitons created in the PF backbone [98]. Using a combination of timedependent many body calculations and experiments, where OLEDs were fabricated using PF2/6 in the presence/absence of a paramagnetic impurity, and in the presence/absence of oxygen, it was shown that the triplet states also play a large role in the 2.1-1.4 eV emission [99]. Dynamical processes such as inter system crossing (ISC) (Figure 6), triplet state quenching, and electron hole recombination processes were modeled in a polyene chain. Even in the absence of any paramagnetic impurity, a significant triplet yield was found [100]. A simple way to understand this picture is that in an electron-hole recombination process, the charge transfer and exchange pathways may result in the mixing of the $T_{1}$ and $S_{1}$ states, thus opening up the $\mathrm{T}_{1}-\mathrm{S}_{0}$ transition, which is typically dipole forbidden. This process may be further facilitated in the presence of a keto defect site.

Pressure dependent PL spectra have been measured from LMW and HMW PF2/6 samples. For the HMW sample, both bulk and thin film samples were measured under pressure [101]. Figure 14 plots the PL spectra at selected values of pressure for powder and thin film samples of HMW PF2/6, measured at room temperature. Due to the higher optical density, the 0-0 vibronic peak is not observed for the powder sample at $0.1 \mathrm{MPa}$. Above $2 \mathrm{GPa}$, the PL spectrum of the powder sample dramatically changes as shown by the spectrum at 2.7 GPa (in red). The vibronic peaks are not discernable beyond this pressure and the backbone emission is completely overwhelmed by an orange emission at $\sim 2.4$ $\mathrm{eV}$. Since the intensity is plotted in normalized units, it is hard to tell from the figure but the intensity of the $2.4 \mathrm{eV}$ emission is much stronger than the blue backbone emission at 0.1 MPa. This is quite atypical of singlet excitons under pressure; in almost all luminescent conjugated molecules and polymers thus far studied, the PL intensity almost always decreases with pressure. The $2.4 \mathrm{eV}$ emission also becomes strong for the thin film sample above $3 \mathrm{GPa}$. There is some hysteresis but upon decreasing the pressure, the blue emission recovers at lower pressures.

At the time when the above experiments were conducted there were no readily available XRD data. The $2.4 \mathrm{eV}$ peak was attributed to keto defects and aggregate emission. However, one must bear in mind that in a closed pressure cell the keto defect sites cannot increase; at most the number of defect sites will remain the same as at 0.1 MPa. Electroluminescence experiments from PF2/6 (at atmospheric pressure) shed some light into the $2.4 \mathrm{eV}$ emission. Additionally, temperature dependent PL and Raman scattering from HMW PF2/6 (Section 5.3) indicate that the sample may undergo a phase transition.

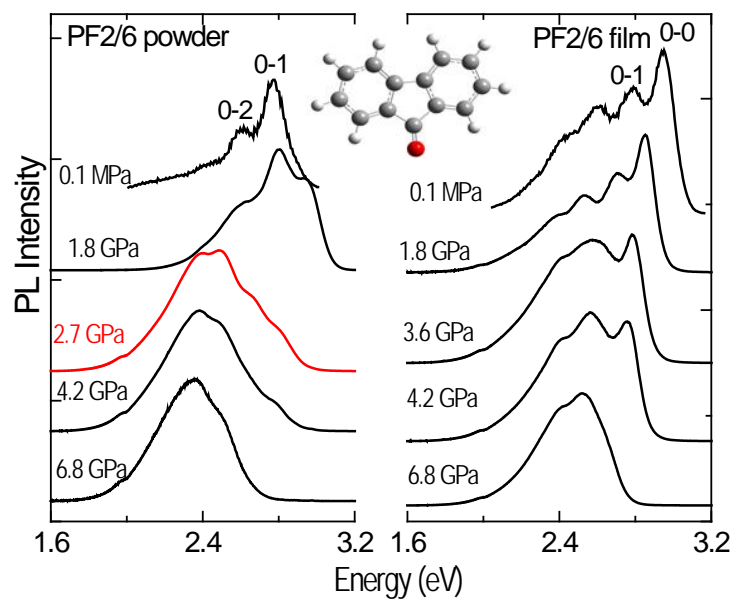

Figure 14. PL spectra of powder and thin film samples HMW PF2/6 at selected values of pressure at room temperature. The inset shows a fluorenone defect.

Unlike PF2/6, PF8 does not exhibit drastic changes in its PL spectrum beyond 2 GPa. Presumably if it was mainly the keto defects that were responsible for the sharp change in PF2/6, we could expect a similar behavior in PF8. To shed some light on the origin of the strong $2.4 \mathrm{eV}$ emission under pressure, we show the electroluminescence from P2/6 OLEDs (Figure 15), where the devices were fabricated under different conditions. 


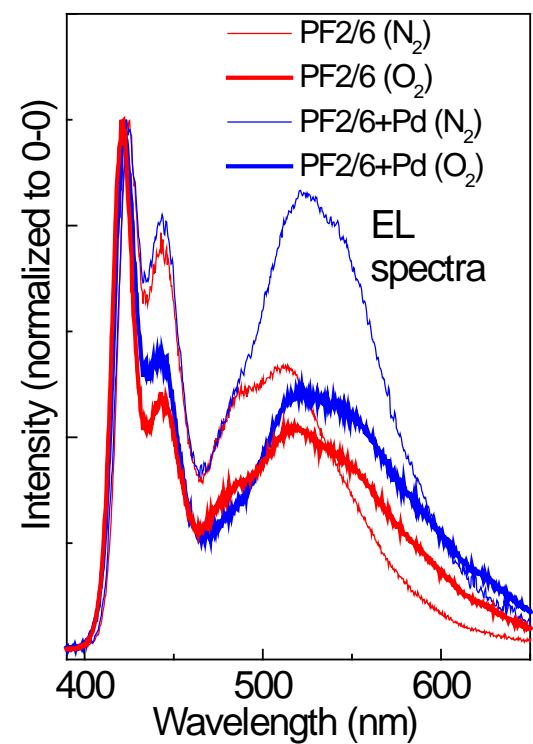

Figure 15 Electroluminescence spectra from PF2/6 OLEDs. Spectra shown in blue included trace quantity of Pd along with PF2/6 and the spectra shown in red is from pristine PF2/6. In both cases, the bold lines correspond to devices fabricated in the presence of oxygen and the thin lines correspond to devices fabricated in a nitrogen atmosphere. Adapted from Ref. [99].

Adding a small quantity of paramagnetic impurity such as Pd in PF2/6 enhances the process of ISC and populates the $\mathrm{T}_{1}$ state, further opening up the $\mathrm{T}_{1}-\mathrm{S}_{0}$ decay channel. This is what is observed in Figure 15. Compared to pristine PF2/6, adding a small amount of Pd enhances the $514 \mathrm{~nm}$ emission. Furthermore, when these devices are fabricated in an oxygen atmosphere, the green emission quenches. This is true both for the pristine and the PF2/6+Pd sample. Clearly if the origin of the green emission was simply due to oxidative defects, one would expect the emission to be enhanced in the presence of oxygen. One way to explain these observations is that the triplet excitons play a role in the low energy emission; the presence of oxygen is known to quench the triplet excitons. Electrophosphorescence has indeed been observed at low temperatures in PFs using time-resolved detection techniques [102].

In light of the electroluminescence data, it is conceivable that the triplet excitons do play a role in the $2.4 \mathrm{eV}$ emission at high pressures. As shown in Section 5.3, Raman scattering above $4 \mathrm{GPa}$ demonstrates signatures of a strong electronphonon coupling. High pressure XRD data now suggests that between 2-4 GPa, HMW PF2/6 undergoes a Hex-Nem phase transition. With a combination of phase change in the HMW PF2/6 sample and charge transfer and exchange pathways, there may be an enhanced mixing of the $S_{1}$ and $T_{1}$ states, which may explain the large change in the PL spectrum of PF2/6 beyond $2 \mathrm{GPa}$.

Contrary to HMW PF2/6, LMW PF2/6 (3/8) which is already in a nematic liquid crystalline phase at $0.1 \mathrm{MPa}$ shows gradual changes in the PL spectra under pressure (Figure 16). Here the nomenclature $3 / 8$ is for the number and weight-averaged molecular weights, which were $3 \mathrm{~kg} / \mathrm{mol}$ and $8 \mathrm{~kg} / \mathrm{mol}$, respectively [44].

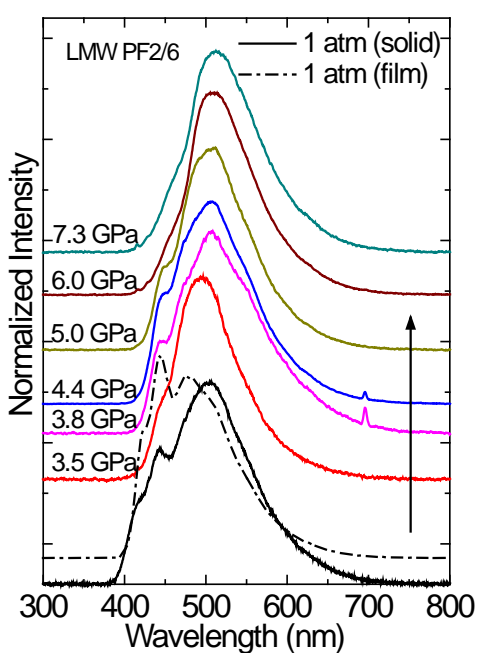

Figure 16. PL data of 3/8 PF2/6 with increasing pressure. Reproduced with permission from [44]. Copyright 2014 Wiley Periodicals, Inc.

The spectrum of 3/8-PF2/6 has nearly the same width as the HMW PF2/6. This implies that the finite polymer size does not play a role in its electronic properties. Should this be the case we would expect a much broader PL spectrum. The PL from the thin film at 0.1 MPa (dashed solid line in Figure 16) shows the $0-0$ vibronic peak at $420 \mathrm{~nm}$ as a shoulder; due to self-absorption the peak intensity is reduced. A higher intensity $0-1$ peak appears at $440 \mathrm{~nm}$. These peaks are seen in the bulk sample as well (black solid line at $0.1 \mathrm{MPa}$ ), which was used in the DAC. Overall, the PL spectrum redshifts but there are no abrupt changes as seen in the HMW PF2/6 sample (Figure 14).

\subsection{Triplet and polaron states}

The weak van der Waals forces in organic semiconductor results in a low dielectric constant; as a consequence the Coulomb and exchange interactions between electrons in these systems are large. The splitting between the lowest excited singlet $\left(\mathrm{S}_{1}\right)$ and triplet $\left(\mathrm{T}_{1}\right)$ levels is of the order of 1 $\mathrm{eV}$. Owing to this large $\mathrm{S}_{1}-\mathrm{T}_{1}$ splitting in organic semiconductors, the triplet states play a major role in the emission mechanism [103].

Phase sensitive photomodulation is a powerful tool to obtain the spectra and lifetimes of excitations of triplet-triplet absorption, intra polaron absorption and charge transfer complexes, not accessible by continuous wave (cw) PL spectroscopy and absorption. Unlike cw spectroscopy, which highlights near band-gap transitions, modulation spectroscopy gives insight into the extended band structure of a solid [104]. Such quasi-steady-state transient photoinduced absorption (PIA) measurement provides opportunities to probe both the emissive and the absorptive states. PIA measurements may further yield signatures of polarons, especially from donor-acceptor blends or defect-induced polymers upon photoexcitation. Transient picosecond high pressure PIA experiments from a PPV derivative have further shed light on the nature of excimers that tend to blueshift with pressure, when the film is ordered [105].

The PIA spectrum is typically measured using a 
mechanically chopped pump beam (laser) and probe beam (tungsten-halogen lamp) in a transmission mode with a lockin amplifier technique [106, 107]. The changes in transmission $(\Delta \mathrm{T})$ divided by the transmission $(\mathrm{T})$ is measured. The signal is usually collected by a photodiode with the electrical output amplified using a preamplifier and split into ac and dc components. The ac component corresponds to $\Delta \mathrm{T}$, and the dc component corresponds to $\mathrm{T}$.

In defect-free conjugated polymers the polaronic signature is difficult to observe optically. MeLPPP which has a planar backbone exemplifies such a defect-free polymer since the torsional degree of freedom is absent between the phenyl rings or between adjacent monomers. Polaron formation and stabilization are favored by the presence of defects. Moreover, since the ISC is quite small in the pristine polymer, the triplet signatures are negligible at room temperature. Figure 17 (a) shows the PIA spectrum from MeLPPP at $0.1 \mathrm{MPa}$ at $77 \mathrm{~K}$ [87]. The $1.3 \mathrm{eV}$ peak corresponds to the triplet-triplet (TT) absorption. The photobleaching $(\mathrm{PB})$ of the absorption is observed at $2.7 \mathrm{eV}$. Only a photo-oxidized sample (where defects are introduced intentionally) shows the signature of the polaron absorption at $2.0 \mathrm{eV}$. This peak is red-shifted for the $3.8 \mathrm{GPa}$ PIA spectrum. PIA under pressure was observed from both as-is and a photo-oxidized MeLPPP samples. The TT and PB peak positions as a function of pressure are shown in Figure 17 (b) and (c). Not surprising, the PB peak shows a pressure coefficient similar to PL and absorption. However, the TT peak red-shifts more slowly compared to the singlet excitonic peaks with pressure. This is attributed to a more localized nature of the triplet excitons compared to the singlet excitons. The polaron absorption peak for the photo-oxidized sample showed a similar pressure coefficient as the PL peaks [87].
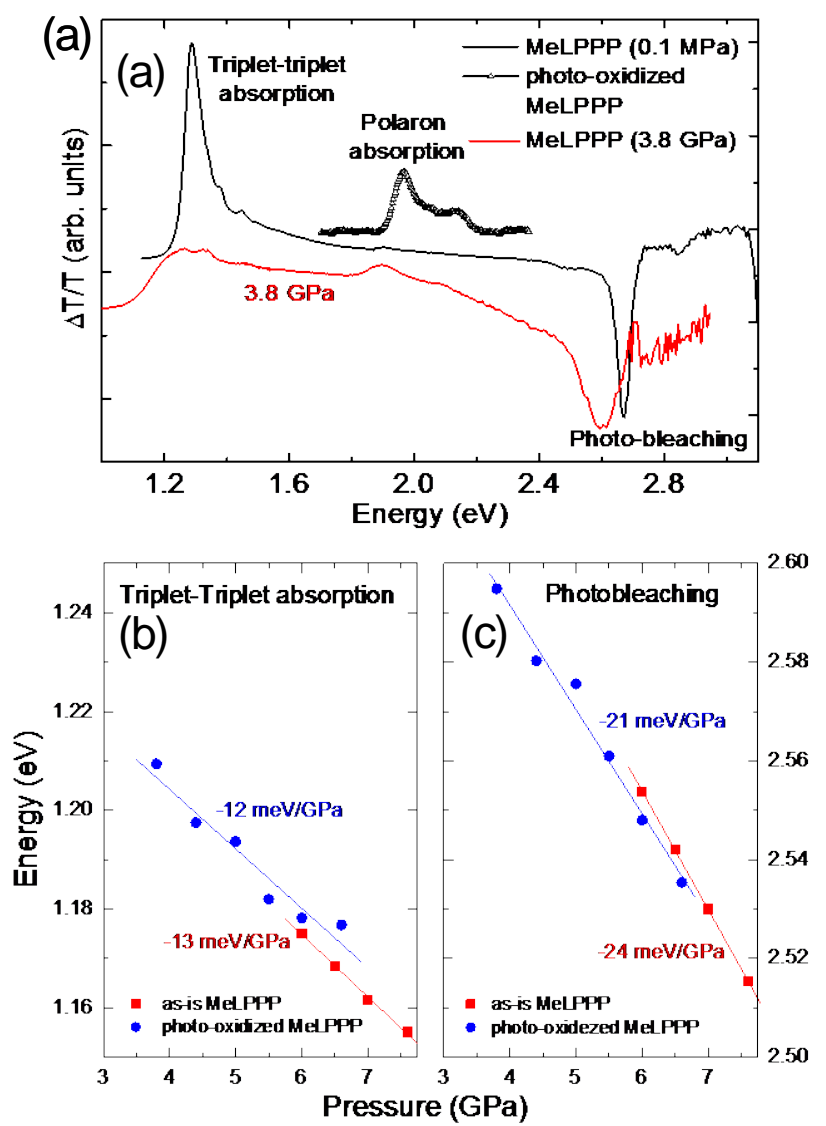

Figure 17. (a) PIA spectrum of MeLPPP (including from a photooxidized film) at $0.1 \mathrm{MPa}$. The red curve is the PIA spectrum from a photo-oxidized MeLPPP film at $3.8 \mathrm{GPa}$. The measurements were at $77 \mathrm{~K}$. (b) and (c) show the triplet-triplet absorption and the photobleaching peak positions for as-is and a photo-oxidized MeLPPP as a function of pressure. Adapted from Refs.[87, 108].

Introduction of trace concentration of Pd or other heavy atom in a polymer backbone opens up the $\mathrm{T}_{1}-\mathrm{S}_{0}$ decay channel since the heavy atom breaks the spin selection rule for emission without the need for change in structural material properties. This has been done for the ladder-type polymer with a diphenyl substitution of the side chain (PhLPPP) where the Pd concentration was less than 200 ppm. In such polymeric systems the $\mathrm{T}_{1}$ state is populated due to an increased ISC at room temperature, allowing the TT absorption to be probed without lowering the temperature. Furthermore, the optical properties such as PL and absorption are almost identical for MeLPPP and PhLPPP. PhLPPP has served as an important system for elucidating the fundamental properties of electrophosphorescence [109], temperature-dependent triplet diffusion [110], and triplet diffusion length [107].

Since both singlet and triplet excitonic levels can be tracked in PhLPPP, it has allowed the study of the singlettriplet splitting energy $\left(\mathrm{S}_{1}-\mathrm{T}_{1}\right)$ under pressure. Figure 18 shows the schematic of the various transitions involving singlet and triplet states in PhLPPP. The electroluminescence spectrum shows a very strong phosphorescence peak at 2.1 $\mathrm{eV}$, which is weakly observed in the fluorescence data [111]. 


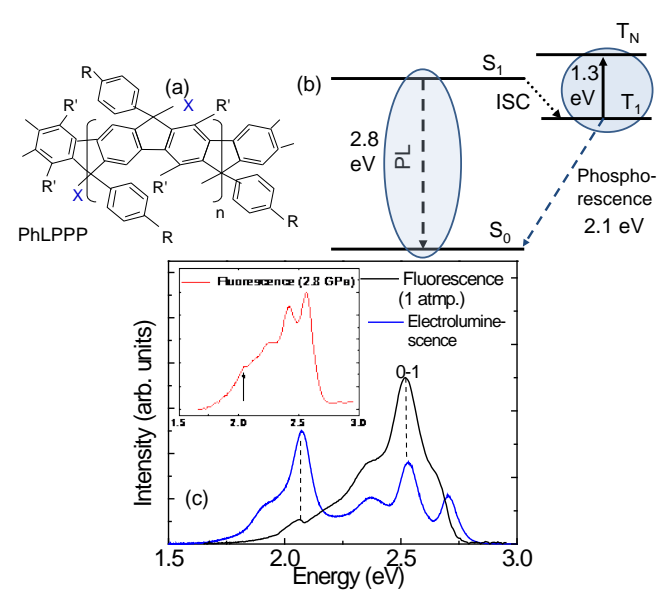

Figure 18 (a) Chemical structure of PhLPPP; X denotes a phenyl group. (b) Schematic of the singlet and triplet states depicting the various transitions. (c) The electroluminescence and fluorescence spectra from PhLPPP at 0.1 MPa. The phosphorescence peak at 2.1 $\mathrm{eV}$ is observed in both spectra. The inset shows the fluorescence spectrum at 2.8 GPa; the arrow denotes the phosphorescence peak. Reproduced with permission from [111]. Copyright 2013 The Institute of Physics.

The high pressure experiments by Paudel et al. were performed with a LMW PhLPPP sample $\left(\mathrm{M}_{\mathrm{n}}=3800 \mathrm{~g} / \mathrm{mol}\right)$ since the triplet signature is stronger compared to a HMW sample. The TT absorption peak is easily discernable at room temperature from PhLPPP due to a higher ISC (Figure 19 (a)), and thus simplifies the experimental condition with a DAC. It is further observed that the LMW sample shows a broader TT absorption peak compared to the HMW sample. The broadened peak arises from a distribution of conjugation lengths due to the configurational degrees of freedom, resulting in a distribution of $\pi$-electron transfer integrals between C-C bonds.
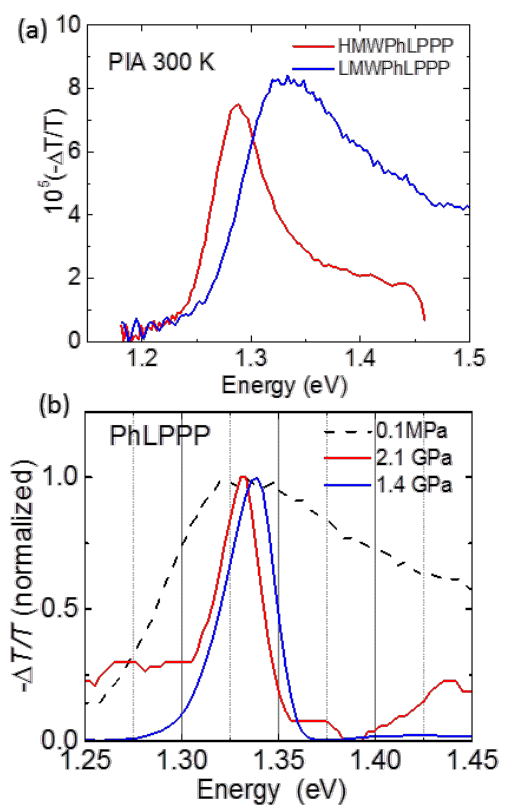

Figure 19. (a) PIA spectra for HMW and LMW PhLPPP at $300 \mathrm{~K}$. (b) PIA spectra of LMW PhLPPP for a few selected values of pressure.

Upon increasing the pressure, the TT peak dramatically narrows at $1.4 \mathrm{GPa}$ and red-shifts with further increase in pressure (Figure 19 (b)). Reduced inter-chain distances of the predominantly amorphous PhLPPP sample upon enhanced pressure could result in reduced conformational freedom resulting in the observed narrowing of the TT absorption peak. Similar to MeLPPP, the TT peak in PhLPPP red-shifts linearly at $-11 \mathrm{meV} / \mathrm{Gpa}$. The intensity of the PIA signal in PhLPPP decreases with pressure. Beyond $4 \mathrm{GPa}$, it is difficult to detect the PIA signal at room temperature.

Table 3. Linear pressure coefficient of optical transitions in PhLPPP.

\begin{tabular}{cc}
\hline Transition & $\begin{array}{c}\text { Pressure coefficient } \\
(\mathrm{meV} / \mathrm{GPa})\end{array}$ \\
\hline 0-0 PL & $-32.4 \pm 2.0$ \\
0-1 PL & $-28.0 \pm 2.0$ \\
Phosphorescence & $-32.0 \pm 0.2$ \\
TT absorption & $-10.7 \pm 0.3$ \\
\hline
\end{tabular}

Table 3 lists the linear pressure coefficient of the various transitions in PhLPPP. The $\mathrm{S}_{1}-\mathrm{S}_{0}$ and $\mathrm{T}_{1}-\mathrm{T}_{0}$ transitions change roughly by the same amount with pressure, indicating that the $\mathrm{S}_{1}-\mathrm{T}_{1}$ splitting does not change, at least up to $4 \mathrm{GPa}$. The lower pressure coefficient of the $T_{1}-T_{N}$ transition in the PIA experiments suggests that the higher-lying triplet states have a more ionic character than the lower $T_{1}$ state. The lifetime of the triplet excitons was further found to decrease with pressure both in PhLPPP [111] and MeLPPP [87].

The $T_{1}-T_{N}$ absorption in PIA and phosphorescence are undetectable beyond $4 \mathrm{GPa}$ (at $300 \mathrm{~K}$ ) in PhLPPP, and combined with the effect that $\mathrm{S}_{1}-\mathrm{T}_{1}$ splitting remains almost a constant within this pressure range, one can safely claim that there is hardly any back transfer of the triplet excitons into the singlet manifold. The main mechanism for the quenching of the triplet excitons, instead, is triplet-triplet annihilation process, where two triplet excitons yield singlet excitons $\left(\mathrm{T}_{1}+\mathrm{T}_{1} \rightarrow \mathrm{S}_{0}+\mathrm{S}_{1}\right)$.

Contrary to the experimental observation of the $S_{1}-T_{1}$ splitting in PhLPPP remaining a constant, first-principles calculations in anthracene show a closing of the $\mathrm{S}_{1}-\mathrm{T}_{1}$ energy gap from $1.22 \mathrm{eV}$ at $0.1 \mathrm{MPa}$ to $0.7 \mathrm{eV}$ at $10 \mathrm{GPa}$ [88]. A few other studies show a change in the exchange energy upon application of pressure. Transient absorption spectroscopy of poly(9,9-dioctylfluorene-co-benzothiadiazole) (F8BT) under hydrostatic pressure shows an increased dielectric constant with pressure, resulting in a delocalization of the singlet excitons [112]. Electron-spin-resonance studies of doped polypyrrole under high pressure show an enhancement of the exchange energy, resulting in decreased spin susceptibility [113]. The behavior of the $S_{1}-T_{1}$ energy gap under pressure still remains an open question. It is conceivable that amorphous polymers such as PhLPPP are different from polycrystalline molecules.

One may naively expect pressure to increase the overlap of the orbitals and hence an increase in the exchange integral. However, the experimental evidence of the unchanged $S_{1}-T_{1}$ energy gap in PhLPPP below 4 GPa, suggests the need for exploring other competing mechanisms that counterbalance the increase in the dielectric constant, if any. The photophysics of triplet excitons is important not only for our understanding of basic excitation/emission properties in 
conjugated polymer, but also for improvement of the performance of optoelectronic devices based on these polymers.

\section{Vibrational states under pressure}

Raman spectroscopy is an extremely powerful, noninvasive tool for characterizing the physical and chemical properties of materials. Typical applications include determining crystalline orientations, characterization of doping levels, stress measurements and study of alloy semiconductors [114]. For organic semiconductors this technique is particularly informative on chemical composition, segmental orientation, conformational distribution and phase identification [115]. The Raman spectra associated with polymer backbones yield structural information. The lower lying vibrations $\left(<200 \mathrm{~cm}^{-1}\right)$ that originate from skeletal deformation modes or interchain interactions throw light on the microscopic structure of the 3dimensional state. Most of these applications emphasize vibrational phonon spectroscopy.

High pressure Raman scattering is a powerful tool for probing molecular interactions in crystalline and amorphous solids. In the harmonic approximation, the longitudinal motion of a mass connected to two springs whose ends are fixed results in a frequency (and thus a force constant, $k$ ) that does not change for a higher compression of the spring. This is the classical Hooke's law: $F=-k x$. The vibrational frequencies of a strictly harmonic solid are independent of compression; pressure-induced changes in the Raman spectrum thus provide insight into the anharmonicity of the solid-state potential. Anharmonicity typically arises from the fact that $k$ depends upon the displacement, $x$.

In 1908, Eduard Grüneisen put forward a relationship between compressibility, thermal expansion, atomic volume, and specific heat of metals [116]. He assumed that the relative change of any vibrational frequency, $v$, is directly proportional to the relative change in volume (V) [117]:

$$
-\frac{\Delta v_{i}}{v_{i}} \times \frac{V}{\Delta V}=-\frac{d \ln \left(v_{i}\right)}{d \ln (V)}=\gamma_{i},
$$

where $\gamma_{i}$ is the volume-independent Grüneisen parameter. Using the above equation, one can further show that the pressure induced vibrational shifts are given by

$$
\frac{v_{i}(P)}{v_{i}(0)}=\left[\frac{V(0)}{V(P)}\right]^{\gamma_{i}}
$$

where $v_{\mathrm{i}}(P)$ is the wavenumber at the applied pressure $(P)$ $V(P)$ is the volume of the bulk solid at $P ; \gamma_{\mathrm{i}}$ is the vibrational mode Grüneisen parameter and is a measure of the anharmonicity in potential energy surface of the specific vibrational coordinate. For crystalline solids, it is a fair assumption that $\gamma_{i}$ is the same for all vibrational modes $\left(\gamma_{i}=\right.$ $\gamma$ ). One can then obtain a bulk thermodynamic definition of $\gamma$ in terms of the volume compressibility, $\chi$, heat capacity, $C_{v}$, and coefficient of expansion of the crystal, $\beta$, given by $\gamma=\beta / \chi C_{v}$. Since the compressibility is usually anisotropic in conjugated polymers and molecules, the mode Grüneisen parameter is expected to be different for different vibrational modes [118].

The broadening of Raman linewidths under pressure is an indication of anharmonic processes. The intrinsic Raman linewidths under pressure are often difficult to extract since non-hydrostatic conditions in a DAC or creation of defects tend to increase the linewidth. Provided other sources of broadening are small, the Raman linewidth in frequency units is proportional to $(2 \pi \tau)^{-1}$, where $\tau$ is the lifetime of the optical phonons. For most crystals an increase in Raman linewidth is observed; Si and Ge show a linear dependence of the linewidth of the optical phonon mode with pressure [119]. The decrease of the phonon lifetime with increasing pressure is attributed to pressure-induced changes in the phonon density of states governing anharmonic decay.

\subsection{Insights into backbone planarity in oligophenyls}

The Raman spectrum of oligophenyls is mainly characterized by four intense modes of $A_{g}$ symmetry. The three predominant ones are observed in the $1100-1700 \mathrm{~cm}^{-1}$ range. Figure 20 compares the Raman spectrum of PHP with other blue-emitting polymers discussed in this review. The three peaks at 1220,1280 , and $1600 \mathrm{~cm}^{-1}$ in PHP are assigned to the in-plane $\mathrm{C}-\mathrm{H}$ bend, $\mathrm{C}-\mathrm{C}$ interring stretch, and the aromatic C-C on-ring stretch modes, respectively. In shorter oligophenyls, the $1600 \mathrm{~cm}^{-1}$ mode is a double peak structure and has been suggested to originate from a Fermi resonance between a fundamental mode and some combination band [120]. The high energy peak in this region is the overtone mode $\left(\mathrm{B}_{1} \otimes \mathrm{B}_{1}\right)$. In PHP, only a weak reminiscence of the overtone mode is seen (red arrow) in contrast to shorter oligophenyls such as terphenyl and quaterphenyl where the overtone mode is comparable in intensity to the C-C stretch peak. In longer chain PPP-type polymers such as MeLPPP, the doublet in the $1600 \mathrm{~cm}^{-1}$ region (Figure 20) is due to a lowering of symmetry.

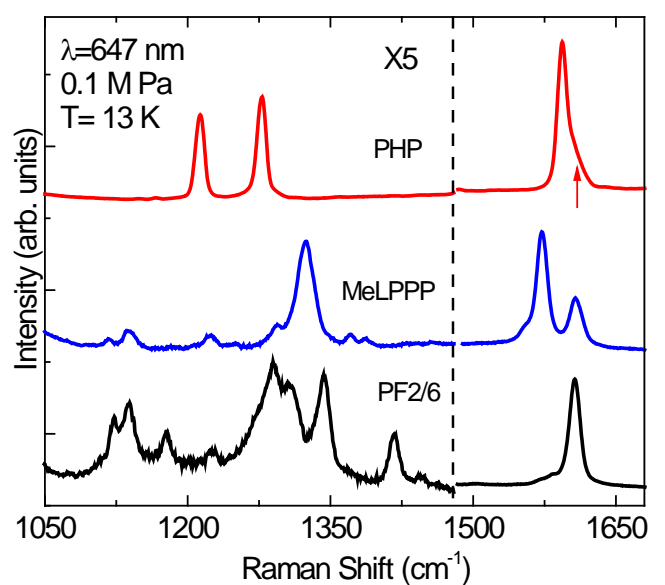

Figure 20. Raman spectra of bulk PHP, MeLPPP and PF2/6 at 0.1 MPa and $13 \mathrm{~K}$. The vertical scale has been expanded by $5 \times$ below $1500 \mathrm{~cm}^{-1}$. Reproduced with permission from [80].Copyright 2004 Wiley Periodicals, Inc.

For MeLPPP and PF2/6, the Raman frequencies in the $1100-1200 \mathrm{~cm}^{-1}$ region are sensitive to side-group 
substitution. The two peaks at $1120 \mathrm{~cm}^{-1}$ and $1137 \mathrm{~cm}^{-1}$ in PF2/6 arise from C-H bending modes of the ethyl-hexyl sidechain. The $1250-1350 \mathrm{~cm}^{-1}$ region shows subtle differences amongst various PF derivatives and LPPPs. These peaks are associated with backbone C-C stretch frequencies, discussed in detail in the next section.

The Raman intensity ratio $\mathrm{I}_{1280} / \mathrm{I}_{1220}$ in para oligophenyls has been found to be a good indicator of the conjugated segments [121-123], and thus also used for monitoring the quality of synthetic routes of PPP systems [124]. $\mathrm{I}_{1280} / \mathrm{I}_{1220}$ versus the number of phenyl rings in oligophenyls is compiled in Figure 21. For biphenyl, $\mathrm{I}_{1280} / \mathrm{I}_{1220} \approx 25$ and for PHP the Raman intensity ratio decreases to $\sim$ unity [33]. The inset shows the Raman spectra of PHP at $0.1 \mathrm{MPa}$ and 2.1 GPa. $\mathrm{I}_{1280} / \mathrm{I}_{1220}$ decreases quite dramatically between $0.1 \mathrm{MPa}$ and $1.5 \mathrm{GPa}$, beyond which it remains a constant. This is an indication of a planarization of the backbone of the molecule where the torsional angle between the neighboring phenyl rings decreases till $1.5 \mathrm{GPa}$, reaching maximum planarity. The Raman data corroborates the photoluminescence spectra of PHP, presented in Section 4.1.1. (Figure 11).

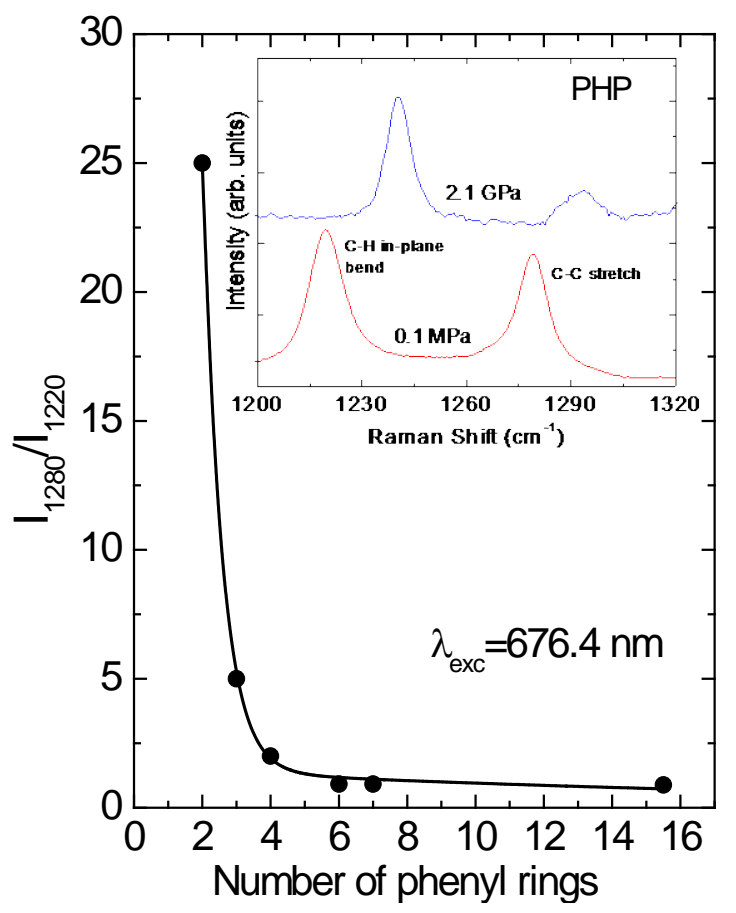

Figure 21. The Raman intensity ratio $I_{1280} / I_{1220}$ versus number of phenyl rings in oligophenyls. The inset shows the Raman spectra of PHP at 0.1 MPa and 2.1 GPa. Reproduced with permission from [33]. Copyright 2001 The American Chemical Society.

Using first principles quantum chemical methods within Albrecht's Raman intensity formalism [125, 126], Heimel et al. have shown the interdependence of $\mathrm{I}_{1280} / \mathrm{I}_{1220}$ in paraoligophenylenes to the oligomer length, planarity, and excitation wavelength [127]. The $1220 \mathrm{~cm}^{-1}$ mode draws its intensity mainly from a transition (virtual) into the lowest excited electronic state. This state can be described by transitions from delocalized occupied molecular orbitals (MOs) into delocalized unoccupied MOs, which are very sensitive to chain length and planarity. As a consequence, the $1220 \mathrm{~cm}^{-1}$ mode is very sensitive to these parameters, experiencing strong conjugation enhancement. A more complex interplay between the vibrational coupling to the delocalized and localized electronic states leads to a less pronounced conjugation enhancement of the $1280 \mathrm{~cm}^{-1}$ mode.

Since the nature of the torsional motion of the phenyl rings in oligophenyls is mainly determined by the intramolecular repulsion between the ortho hydrogens and a resonance interaction of the $\pi$ electron system, high pressure is a mechanism of tuning these interactions. Under atmospheric conditions, the potential energy of two neighboring phenyl rings as a function of the torsional angle is "W"-shaped and the libration of the phenyl rings occur between the two minima of this potential. At elevated pressures, the potential well becomes more "U"-shaped [33].

Using DFT, we calculate the Raman spectrum of a PHP molecule for the optimized geometry (where the backbone is non-planar) and for a planar geometry, where the rings were forced to be at $180^{\circ}$. Details of the theoretical methodology including correlation between theoretical Raman intensities and experimental data are found in Refs. [128, 129]. The theoretical Raman spectra of PHP are shown in Figure 22; the Raman intensity (scattering cross section) is normalized to the $1600 \mathrm{~cm}^{-1}$ peak. Since the calculations are in the gas phase, it is not a surprise that the frequencies (Stokes) are shifted to higher energies compared to the experimental data. The relative intensity ratio of the $\mathrm{C}$ - $\mathrm{C}$ stretch mode to the $\mathrm{C}$ $\mathrm{H}$ bend mode $\left(\mathrm{I}_{1280} / \mathrm{I}_{1220}\right)$ shows a similar trend to the experimental data (inset of Figure 21), further confirming that high pressure changes the backbone conformation of PHP to a more planar geometry.

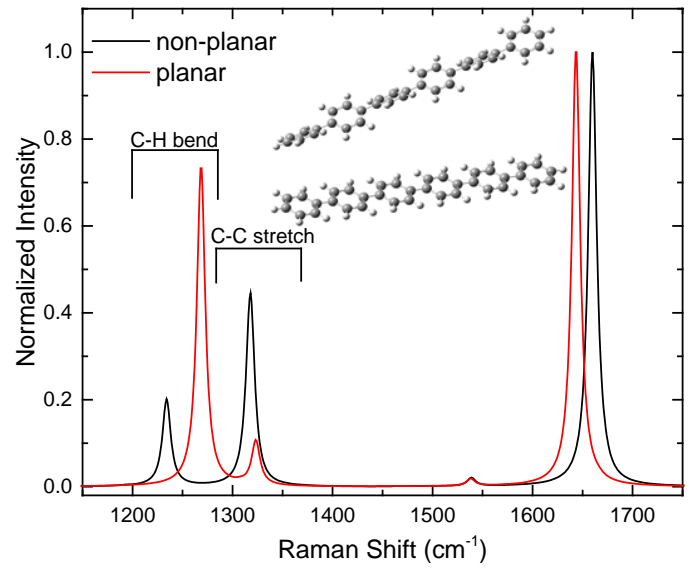

Figure 22. Calculated Raman spectrum of a PHP molecule for an optimized (non-planar) and planar geometries. The inset shows the optimized and planarized geometries of the phenyl rings.

Smaller oligophenyls such as $p$-terphenyl (3P) and $p$ quaterphenyl (4P) under pressure show a similar trend of the $\mathrm{I}_{1280} / \mathrm{I}_{1220}$ Raman intensity ratio as PHP - a rapid decrease till planarization of the backbone is achieved, and then a fairly constant ratio (Figure 23). The pressure at which planarization occurs is $\sim 1 \mathrm{GPa}$ in smaller oligophenyls [130]. When the experiments are conducted at fairly low temperatures, a discontinuity in the Raman frequency position of the $1220 \mathrm{~cm}^{-1}, 1280 \mathrm{~cm}^{-1}$, and $1600 \mathrm{~cm}^{-1}$ modes is 
also observed at the planarization pressure.

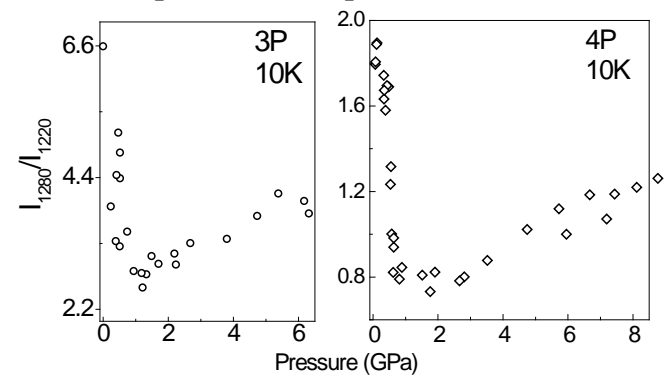

Figure 23. The Raman intensity ratio $\mathrm{I}_{1280} / \mathrm{I}_{1220}$ in $3 \mathrm{P}$ and $4 \mathrm{P}$ as a function of pressure. The measurements were performed at $10 \mathrm{~K}$ with $647.1 \mathrm{~nm}$ as the excitation wavelength. Reproduced with permission from [130]. Copyright 2004 Wiley Periodicals, Inc.

Planarization of oligophenyls under pressure has also been observed by infrared (IR) spectroscopy [131, 132]. Several IR absorption peaks associated with the out-of-plane $\mathrm{C}-\mathrm{H}$ bend modes in $3 \mathrm{P}$ and $4 \mathrm{P}$ disappear when the molecule planarizes. The disappearance of the IR modes has been
attributed to a change from a low symmetry non-planar configuration $\left(C_{2 h}\right.$ in $\left.3 \mathrm{P}\right)$ to a higher symmetry $\left(D_{2 h}\right.$ in $\left.3 \mathrm{P}\right)$ of the molecule, when fully planarized.

\subsection{Mode Grüneisen parameter}

Typically, all Raman frequencies in conjugated polymers and molecules harden as a function of pressure. The Raman frequency positions of the $\mathrm{C}-\mathrm{C}$ and $\mathrm{C}-\mathrm{H}$ modes under pressure for PFs will be discussed in the next section. Here we simply comment on the mode Grüneisen parameter for the Raman modes in PHP. As stated earlier, $\gamma_{i}$ relates to the bulk thermodynamic properties for crystalline solids. Ideally, to determine $\gamma_{i}$, along with a change in the vibrational frequency with pressure, the change in the volume of the crystal with pressure is also required. Since XRD under pressure from conjugated molecules and polymers has been scarce as a result of the challenges involved with such experiments, the volume dependence under pressure is not known for most systems. The Grüneisen parameters for the $1220 \mathrm{~cm}^{-1}, 1280$ $\mathrm{cm}^{-1}$, and $1600 \mathrm{~cm}^{-1}$ Raman modes of PHP were ascertained using the change in volume of $p$-terphenyl under pressure [133].

Figure 24 shows a plot of $\log \left[v_{i}(P) / v_{i}(0)\right]$ versus the change in volume under pressure for the three Raman modes in PHP beyond 1.5 GPa, i.e., after the molecule is planarized. Using a linear fit for all three modes, it is seen that none of the fits go through the origin, as one would expect if Eq. 6 were valid. Linear regression analysis for all three lines in Figure 24 (where the intercepts are nonzero) yield a value of $\sim 0.09$ for the slope. The deviation from Eq. 6 suggests that the intramolecular vibrational modes in PHP are associated with volume-dependent Grüneisen parameters. In other words, the volume ratio $(V(P) / V(0))$ for the three modes is different. Since the $\mathrm{C}-\mathrm{H}$ bend mode at $1220 \mathrm{~cm}^{-1}$ is an out of plane motion compared to the $\mathrm{C}$ - $\mathrm{C}$ stretch modes, it is conceivable that different compressibilities along the chain and perpendicular to the chain result in volume-dependent Grüneisen parameters. Such a behavior is observed in polycarbonate, where the volume dependence of the Grüneisen parameters has been attributed to a difference between the local volume compression relative to that of the bulk [118].

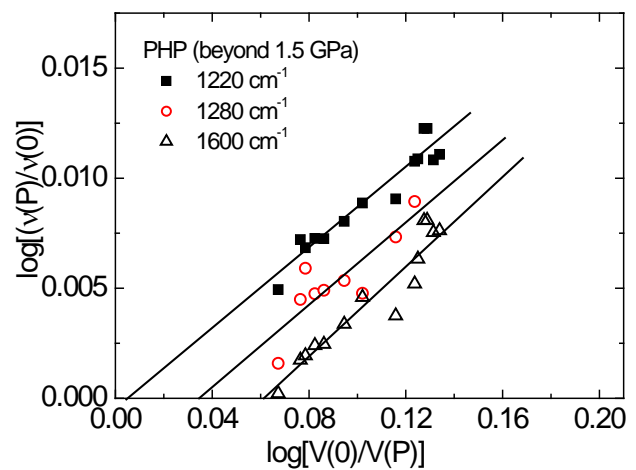

Figure 24. A log-log plot of the wavenumber ratio $v_{i}(\mathrm{P}) / v_{i}(0)$ versus the volume ratio $\mathrm{V}(\mathrm{P}) / \mathrm{V}(0)$ for three Raman active modes in PHP beyond 1.5 GPa. Reproduced with permission from [33]. Copyright 2001 The American Chemical Society.

Given the close connection between the thermodynamic properties of a solid and vibrational frequencies as a function of pressure, high pressure Raman scattering has been a vital tool for estimating compressibility and other anharmonic properties of diamond-like crystals and perovskite structures [134-137]. Unfortunately, for conjugated organic polycrystalline and amorphous materials, high pressure Raman scattering has not been used a whole lot to predict the thermodynamic properties, mainly due to a concomitant lack of x-ray data at high pressures. For a polymorph of diphenyl oxadiazole crystal, Raman scattering and XRD under pressure revealed its bulk modulus and pressure derivative to be 8.6 GPa and 7.2, respectively, and the mode Grüneisen parameters for the Raman modes varied between 0.02 and 2.9 [138].

There has been an on-going interest in the optical properties of polyatomic crystals, minerals, and hydrocarbons under pressure from the geophysical point of view [139-141]. The mode Grüneisen parameter has been redefined for polyatomic crystals to allow for the presence of the functional groups, and has been found to work well for spinel and olivine group minerals [142]. The revised formula uses the polyhedral bulk moduli appropriate to the particular atomic motion associated with each vibrational mode.

With recent interest in high pressure Raman scattering and optical spectroscopic experiments from 2D materials [143, 144], there is perhaps even a greater need for high pressure XRD measurements from soft matter. A complete picture of anharmonicity, thermal properties, validity of the Grüneisen parameter in conjugated molecules and polymers, thus far, is still lacking.

\subsection{Raman spectra of PF2/6 and PF8 under pressure}

The application of Raman scattering in PFs is particularly useful for resolving ambiguities regarding chain conformations and crystalline phases in PFs. The Raman spectrum of PF is characterized by numerous intramolecular 
C-C/C-H stretch and bend modes spanning from 100 to 1600 $\mathrm{cm}^{-1}$, as shown in Figure 25. Similar to other phenyl substituted molecules, the Raman peaks in the $1600 \mathrm{~cm}^{-1}$ region arises from an intraring $\mathrm{C}-\mathrm{C}$ stretch frequency and is best fit with two peaks: an overwhelmingly dominant peak at $1605 \mathrm{~cm}^{-1}$ and at least one or two weak peaks in the range of $1570-1600 \mathrm{~cm}^{-1}$. These weaker peaks correspond to a breathing motion of the pentagon within the monomer. The Raman frequencies in the $1250-1350 \mathrm{~cm}^{-1}$ region, which are quite similar in PF8 and PF2/6, are associated with the backbone $\mathrm{C}-\mathrm{C}$ stretch motion; the peak at $1342 \mathrm{~cm}^{-1}$ being the $\mathrm{C}-\mathrm{C}$ stretch mode from within the monomer unit. The $\mathrm{C}$ C stretch mode between adjacent monomers appears at slightly lower frequencies $\left(\sim 1300 \mathrm{~cm}^{-1}\right)$. The $1257 \mathrm{~cm}^{-1}$ corresponds to a C-H bend type motion.

Frequencies in the $1100-1200 \mathrm{~cm}^{-1}$ region are sensitive to side-group substitution; the 1120 and $1135 \mathrm{~cm}^{-1}$ peaks mainly arise from the $\mathrm{C}-\mathrm{H}$ bending modes (either local or between phenyl units). Crystalline fluorene has a strong peak at 743 $\mathrm{cm}^{-1}$, corresponding to the $747 \mathrm{~cm}^{-1}$ fundamental of a biphenyl with $A_{1}$ symmetry, and thus provides sensitivity of this vibration to the planarity of the ground state [128, 145]. In PFs, this frequency is slightly down shifted to $735 \mathrm{~cm}^{-1}$.

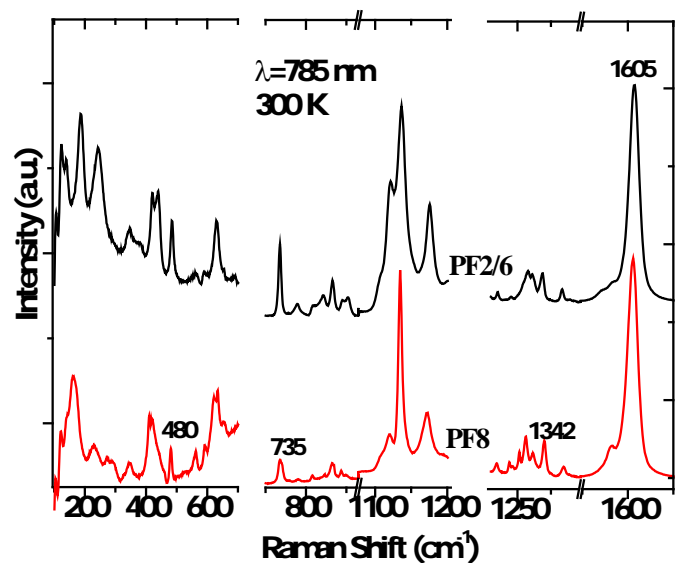

Figure 25. Raman spectra of PF2/6 and PF8 at 0.1 MPa (300 K).

The low frequency Raman peaks in the $100-700 \mathrm{~cm}^{-1}$ range originate mainly from the side chains. This region is thus an extremely sensitive indicator of both side chain composition and ordering. The peak at $480 \mathrm{~cm}^{-1}$ corresponds to the ring torsion mode and is seen in all PFs. Since the Raman peaks around $600 \mathrm{~cm}^{-1}$ and in the range $100-400 \mathrm{~cm}^{-1}$ are predominantly from alkyl side chains, they show different signatures in PF8 and PF2/6. This region, in particular, is very sensitive to the different conformations in PF8: $\mathrm{C}_{\alpha}, \mathrm{C}_{\gamma}$, and $\mathrm{C}_{\beta}$, as discussed in Section 4.1.1. The $\mathrm{C}_{\alpha}$ conformation, attributed with the aaa type di-octyl side chain, shows a longitudinal accordon motion (LAM) with its odd orders which are allowed in Raman scattering. LAM1 occurs at $130 \mathrm{~cm}^{-1}$ in $\mathrm{C}_{\alpha}$ PF8 [128]. Detailed Raman scattering experiments from a series of fluorene oligomers distinguishing the three phases, $\mathrm{C}_{\alpha}, \mathrm{C}_{\gamma}$, and, $\mathrm{C}_{\beta}$, were measured by Tsoi and Lidzey [146]. PF2/6 with its branched alkyl side chain does not show any LAM frequencies.

Some of the more interesting features in the $1300 \mathrm{~cm}^{-1}$ range, which belong to the backbone Raman active modes in the PFs, cannot be tracked in a DAC. The strong $1330 \mathrm{~cm}^{-1}$ Raman peak from diamond completely swamps this region. Furthermore, the low energy region of the Raman spectrum is difficult to measure with a DAC even in the off resonance condition (with excitation energy at $785 \mathrm{~nm}$ ), since at high pressures the PL is red-shifted and the tail of the PL spectrum overwhelms the Raman spectrum.

Being one of the strongest peaks, the $1600 \mathrm{~cm}^{-1}$ Raman peak of PFs under pressure has been well studied. As it is an intraring stretch mode, this frequency is not particularly sensitive to the various conformations in PFs. In PF2/6 (both low and high mol. wt.) and PF8 the pressure coefficient is linear and ranges between $5-7 \mathrm{~cm}^{-1} / \mathrm{GPa}$. Figure 26 shows the Raman spectrum of PF8 in the $1600 \mathrm{~cm}^{-1}$ region. Along with an as-is sample (which is predominantly in the $\beta$ phase), a TC PF8 sample was also measured under pressure [90]. The TC sample is mainly in the $\alpha$ phase. The pressure coefficient of the $1600 \mathrm{~cm}^{-1}$ peak is the same for both phases, highlighting the insensitive nature of this vibration to the backbone conformation. Corroborating the PL data of PF2/6 (shown in Figure 14), the Raman spectra under pressure shows asymmetric line shapes beyond $4 \mathrm{GPa}$, which we discuss in the next section.
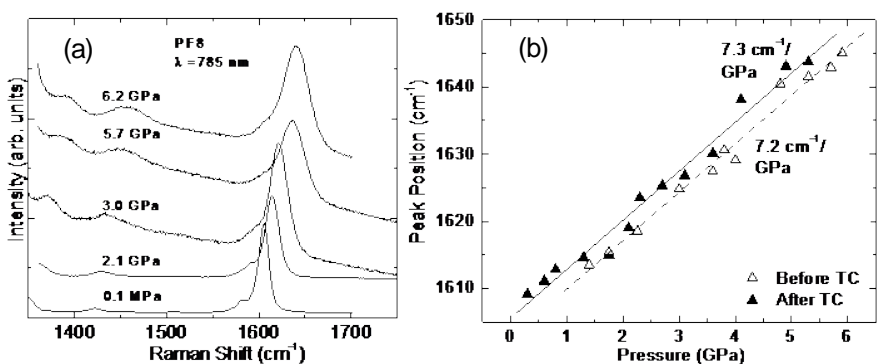

Figure 26. (a) Raman spectra of the $1600 \mathrm{~cm}^{-1}$ region in as-is PF8 at selected values of pressure. (b) The peak position of the $1605 \mathrm{~cm}^{-1}$ Raman peak as a function of pressure for the as-is (before TC) and thermally cycled (after TC) PF8. Adapted from Ref. [90].

The $1135 \mathrm{~cm}^{-1}$ Raman peak in as-is and TC PF8 samples show slightly different pressure coefficients. The origin of this peak is from a C-H in-plane bend motion along with a ring distortion. For a non-planar conformation, the motion splits into two or more vibrations. The pressure coefficients of the $1135 \mathrm{~cm}^{-1}$ Raman peak in the as-is and TC PF8 samples are 1.6 and $2.9 \mathrm{~cm}^{-1} / \mathrm{GPa}$, respectively [90]. The $1135 \mathrm{~cm}^{-1}$ broadens with pressure both in as-is PF8 and LMW PF2/6 (shown in Figure 27), which could be an indication of a reduced torsion angle between adjacent repeat units, consistent with the XRD data presented in Section 6. 


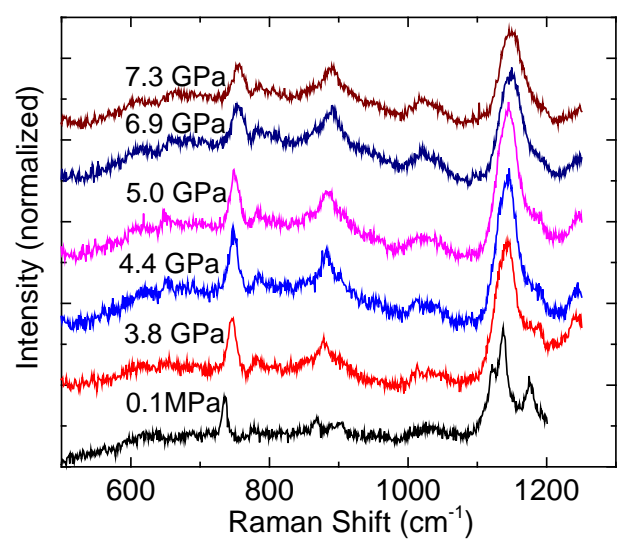

Figure 27. Raman spectra of LMW PF2/6 showing the $600-1200 \mathrm{~cm}^{-1}$ region at selected values of pressure. Reproduced with permission from Ref. [44]. Copyright 2014 Wiley Periodicals, Inc.

\subsubsection{Enhanced electron-phonon interaction in PF2/6}

Asymmetric lines shapes in the Raman frequencies are often observed in heavily doped semiconductors [147-149]. The energy continuum (due to defects or doping) overlaps with the discrete Raman active optical phonon resulting in an interference between the phonon and electronic transitions. The line shape of the phonon is modified to an asymmetric Breit-Wigner Fano (BWF)-type, which is characteristic of the strong electron-phonon discrete-continuum interaction [150]. In carbon based materials, BWF line shape seen in the Gband of graphene [151] has been theoretically modeled and attributed to an interference between the electron-hole excitation spectra and the discrete phonon [152]. The Raman spectra of high $\mathrm{T}_{\mathrm{c}}$ superconductors are often characterized by BWF line shape, where the nature of the asymmetry yields information on the superconducting gap [153]. As an example, we show the first-order Raman spectra (TO phonon) of pure $\mathrm{Si}$ and p-doped $\mathrm{Si}$, measured with an excitation wavelength of $785 \mathrm{~nm}$ in Figure 28. The asymmetric line shape is clearly seen for p-doped $\mathrm{Si}$.

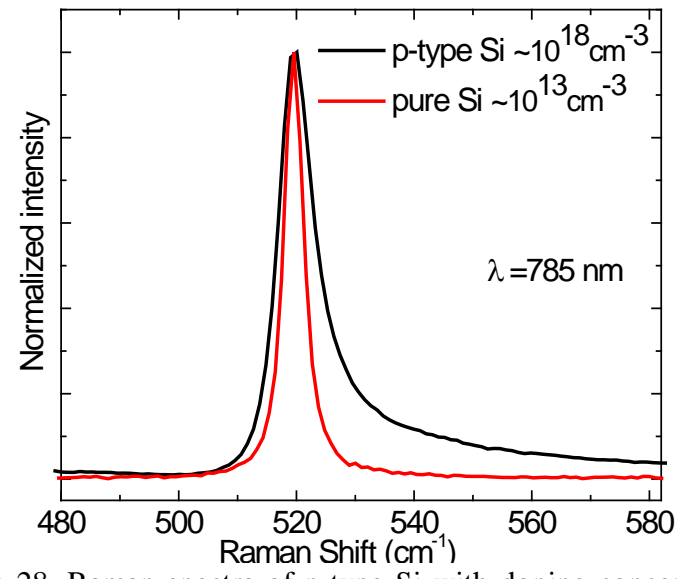

Figure 28. Raman spectra of p-type Si with doping concentration of $10^{18} \mathrm{~cm}^{-3}$ and intrinsic Si.

The BWF line shape is given by:

$$
I(\omega)=I_{0} \frac{\left[\frac{\left(\omega-\omega_{0}\right)}{q}+\Gamma\right]^{2}}{\left(\omega-\omega_{0}\right)^{2}+\Gamma^{2}},
$$

where $\omega_{0}$ is the discrete phonon frequency and $\Gamma$ is the width of the resonant interference between the continuum and discrete scattering channels. The asymmetry parameter (1/q) depends upon the average electron-phonon matrix, $M$, and the Raman matrix elements between the ground and excited states. The broadening parameter is expressed in terms of the electron-phonon matrix and the combined density of states for the electronic transitions, $D(\omega)$ :

$$
\Gamma=\pi M^{2} D(\omega)
$$

where $q=-\left(\omega-\omega_{0}\right) / \Gamma$. The spectral function reveals an anti-resonance close to the value of the phonon frequency.

As seen in Figure 26, the line-shape of the $1600 \mathrm{~cm}^{-1}$ Raman peak at higher pressures in PF8 shows some asymmetry. This is greatly enhanced in bulk PF2/6 (HMW) and is consistent with the large change in the PL spectrum as shown in Figure 14. We note that in the rest of this section, the high pressure Raman scattering data presented is mainly from a HMW PF2/6 sample.

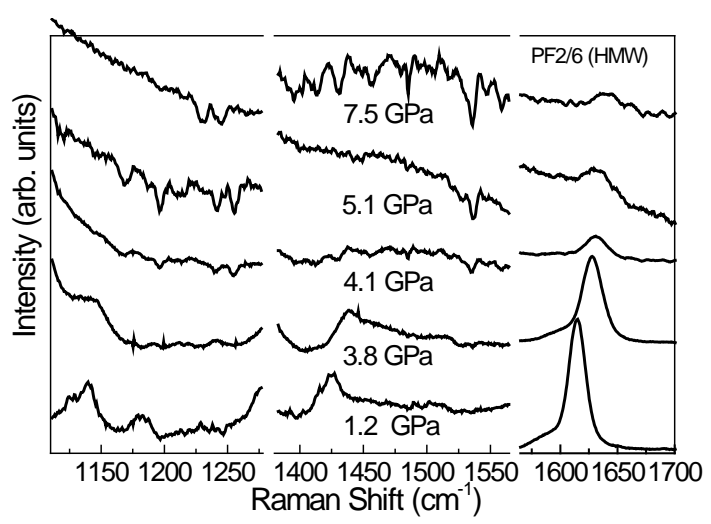

Figure 29 Raman spectra of PF2/6 at selected pressures. Each frequency region has been scaled individually. Reproduced with permission from Ref. [101]. Copyright 2003 The American Physical Society.

Figure 29 shows the Raman spectra from PF2/6 at different pressures. The diamond peak at $1330 \mathrm{~cm}^{-1}$ has been removed for clarity. As the pressure is increased, the background increases and as a result many of the weaker Raman peaks cannot be tracked at high pressures. The region below $1300 \mathrm{~cm}^{-1}$ is observable up to $2 \mathrm{GPa}$ and the $1417 \mathrm{~cm}^{-1}$ is observed up to $4 \mathrm{GPa}$. Above $4 \mathrm{GPa}$, the asymmetry of the $1600 \mathrm{~cm}^{-1}$ peak becomes substantial, beyond which fitting this peak to a Lorentzian line shape becomes difficult. A BWF line shape gives an accurate fit. Along with the asymmetry of the $1605 \mathrm{~cm}^{-1}$ peak, new peaks exhibiting antiresonance effects appear near $1230 \mathrm{~cm}^{-1}$ and $1530 \mathrm{~cm}^{-1}$. Some of them are IR peaks that become Raman active. The appearance and disappearance of these peaks with cycling the pressure are very consistent. The asymmetry and the antiresonant peaks are typical of a BWF interaction.

Figure 30 shows the asymmetry parameter (1/q), obtained by fits to the $1600 \mathrm{~cm}^{-1}$ peak at each pressure, using Eq. 7. The parameter is relatively small between $0.1 \mathrm{MPa}$ and $3 \mathrm{GPa}$; beyond $4 \mathrm{GPa}, 1 / \mathrm{q}$ varies between -0.3 to -0.6 . The negative sign for q suggests that the energy of the electronic continuum lies below the discrete phonon frequency of 1600 
$\mathrm{cm}^{-1}(0.2 \mathrm{eV})$. The inset shows a sample BWF fit at $4 \mathrm{GPa}$. The linewidth shows a square law dependence with pressure $\left(\Gamma \propto P^{2}\right)$. For details, see Ref. [101]. Moreover, the frequency position shows a linear dependence with pressure. One expects an additional shift in phonon frequency due to the BWF interaction (which is due to the real part of the selfenergy). In materials such as doped $\mathrm{Si}$, where the BWF interaction could be tuned by uniaxial stress, the real part of the self-energy was determined by comparing with a pure material[147]. Such a comparison is difficult in organic semiconductors such as PF2/6 due to a lack of a defect-free but identical material.

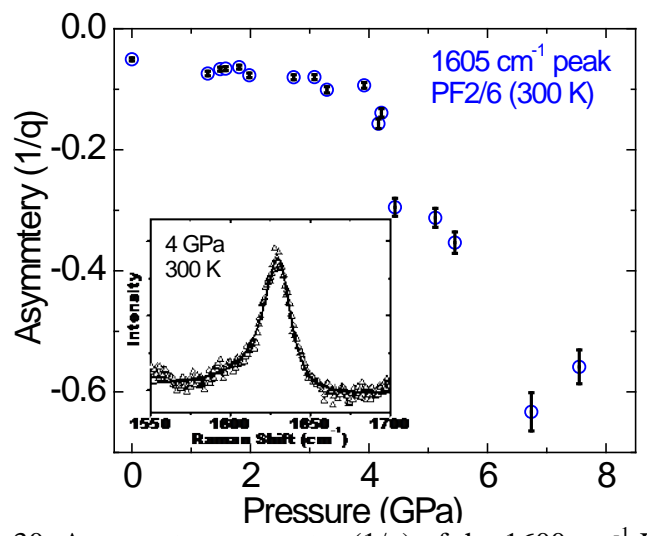

Figure 30. Asymmetry parameter (1/q) of the $1600 \mathrm{~cm}^{-1}$ Raman peak, obtained from a BWF line shape fits, as a function of pressure. The inset shows a sample fit at $4 \mathrm{GPa}$. Reproduced with permission from Ref.[101]. Copyright 2003 The American Physical Society.

What is then the origin of the electronic continuum for the BWF interference in PF2/6? Wide-angle X-ray scattering measurements (Section 6), which were conducted almost a decade later, allude to a hexagonal to nematic phase transition between 2-4 GPa in HMW PF2/6 along with a partial backbone planarization. Such structural changes could induce a change in the electronic density of states arising from aggregate and defect emission. However, the exact origin of the BWF interference in PF2/6 upon increasing pressure is still an open question. Simultaneous Raman and x-ray scattering in PFs by adding additional defects (including trace quantities of heavy atoms to enhance the spin-orbit coupling) could reveal the nature or the electronic density at elevated pressures.

\section{Structure under pressure}

In this section we illustrate selected structural findings deduced from X-ray scattering and related modelling studies. We will first discuss three structurally related materials classes - $\pi$-conjugated oligomers and crystals, $\pi$-conjugated polymers and rigid rod polymers. After this, we focus on the details of polyfluorene PF2/6 whose ambient structure and pressure driven optical properties have been introduced earlier.

We point out that while $\pi$-conjugated oligomers and polymers share the emission properties, their structural similarity is limited to the intramolecular factors. Oligomers are monodisperse and rigid with well-defined structural transitions. They are usually highly crystalline with small unit cells. Polymers are polydisperse with a limited persistence length and smooth transitions with significant hysteresis. The degree of order tends to be much lower and long range order between main chains is usually separated by disordered side chains.

Much of the structural characteristics of $\pi$-conjugated polymers stems from their stiff backbone. This leads to lyotropic and thermotropic liquid crystallinity and distinctive macroscopic alignment of polymers and/or polymer crystallites which yields polarized emission also under pressure (see a rare example for polarized emission shown for compressed poly( $p$-phenylene vinylene) PPV by Morandi et al. [154]). For this reason, $\pi$-conjugated polymers are likened to rigid rod polymers (whether $\pi$-conjugated or not) with stiff backbone and to hairy-rod polymers with stiff backbone and flexible side chains also under pressure. Similarly, the selforganization and phase behavior of hairy-rods [155] provides a good starting point to consider structure of $\pi$-conjugated polyfluorenes [43].

Structural studies allow system characterization in terms of compressibility as

$$
\kappa=\lim _{P \rightarrow 0} \frac{\partial \ln d}{\partial P}=c\left(1-\frac{d}{d_{0}}\right),
$$

where $c$ is a constant, and $d_{0}$ and $d$ are the initial and saturated distances in the system at ambient and infinite pressures. Compressibility can be deduced from the unit cell parameters determined against the pressure. However, as noted by Boldyreva [57], the bulk compressibility is not informative for highly asymmetric systems but one should rather follow anisotropy of structural strain as

$$
\varepsilon_{(h k l)}=\frac{\Delta d}{d_{0,(h k l)}},
$$

where $d_{0,(h k l)}$ is the spacing at ambient pressure and $\Delta d$ the variation of spacing at elevated pressure.

\subsection{Structural analogues}

\subsection{1 $\pi$-Conjugated molecules}

XRD combined with modellings such as DFT based methods has been used to solve structures of diverse aromatic or heterocyclic molecules molecules and $\pi$-conjugated oligomers such as indole [156], diphenyl-1,2,3.oxadiazoles [157], or bis-benzene-1,2-dithiolato crystals [158], oligoacenes including anthracene [159], pentacene, and tetracene [160] as well as oligo $p$-phenylenes including biphenyl [161] and terphenyl [86]. While the structural details vary from molecule to molecule, these studies demonstrate two factors important for compressed $\pi$ conjugated systems - anisotropy and planarization.

First, there is high anisotropy both in structure and in compression. Second, if the molecules allow internal rotations (like in the case of $p$-phenylenes), it is important to monitor the torsion angle $\tau$ between planar moieties and thus 
the planarity of the whole molecule. These features should be followed in the polymer studies as well.

We note that fluorene under pressure makes a connection between $\pi$-conjugated molecules and PFs. At the ambient conditions, fluorene is crystalline with orthorhombic unit cells consisting of four herringbone positioned molecules [162]. This structure can be further modified by alkyl chains as illustrated by the structure of dioctylfluorene by McFarlane et al. [163] making an obvious analogue to the alkyl substitution of polymers.

Figure 31 shows XRD curves of compressed fluorene as reported by Heimel et al. [92]. The data illustrate the known orthorhombic phase at normal pressure and a distinctive phase transition from the herringbone structure to the $\pi$ stacked high pressure phase at around 3.6 GPa. This is a prime example of $\pi$-conjugated molecules manifesting not only a gradual compression of an existing phase but a whole new high pressure phase.

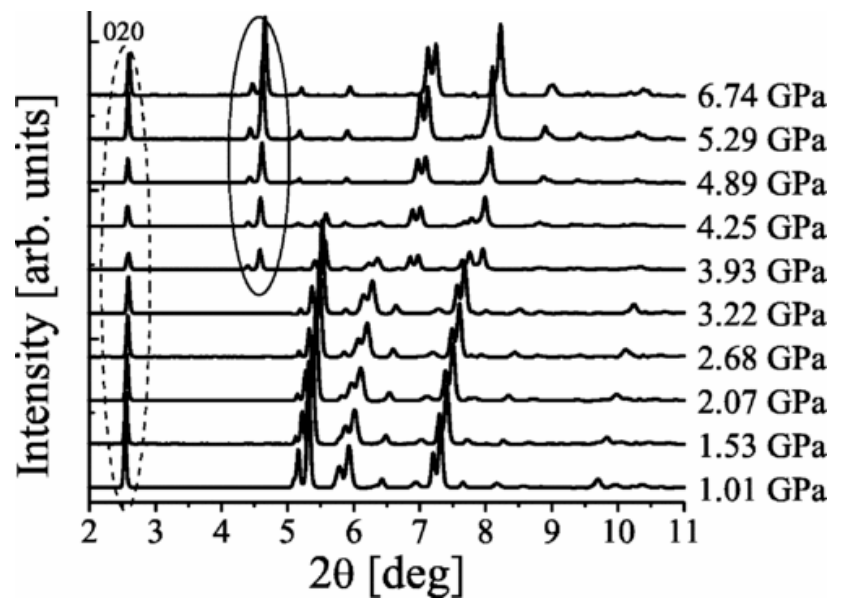

Figure 31. XRD curves of fluorene crystal with increasing pressure. A dashed ellipse illustrates 020 reflection associated with the fluorene layers. A solid ellipse illustrates new phase between $3.2 \mathrm{GPa}$ and 3.9 GPa. Reproduced with permission from [92]. Copyright 2006 The American Physical Society.

\subsection{2 $\pi$-Conjugated polymers}

While there are several structural studies of $\pi$-conjugated molecules, less attention has been placed on $\pi$-conjugated polymers. High pressure XRD studies of $\pi$-conjugated polymers were pioneered using PA [164-166]. At the ambient conditions, trans-PA assumes a space group $\mathrm{P} 21 / \mathrm{n}$ in a monoclinic structure where the chains define the polymer caxis [167]. Pressure lowers the two-dimensional space group symmetry. Furthermore, like with $\pi$-conjugated molecules, the interchain compressibilities are high and anisotropic taking values $8.3 \cdot 10^{-3} / \mathrm{kbar}$ and $3.8 \cdot 10^{-3} / \mathrm{kbar}$ along a- and baxes, respectively [164].

These studies were followed by XRD studies of polythiophenes [168, 169]. The ambient structures of alkylsubstituted polythiophenes are well-known [170]. Poly(3octylthiophene) (POT) takes an orthorhombic layered structure of backbones and side chains whereby the a-axis defines the layer normal, b-axis the backbone stacking and caxis the backbone itself. This is seen by XRD curves showing distinctive $h 00$ peaks and broader 010 (also indexed as 020) peak on top of the amorphous halo as well as weak but sharp 001 at a wider angle [171].

POT and poly(3,3'-dioctyl-2,2'-bithiophene) change their color from red to yellow with increasing pressure. Mårdalen et al. studied the origin of this effect using XRD when increasing the hydrostatic pressure up to $10 \mathrm{GPa}[168,169]$ In agreement with the simulations of Corish et al. [172, 173], it was shown that the distance between neighboring chains decreases and the planarity of lamellar POT increases with increasing pressure and that this is related to a pressure induced optical transition. It was shown, moreover, that poly(3,3'-dioctyl-2,2'-bithiophene) experiences a reduction in the chain twist angle at high pressure, which explains a similar optical effect. Figure 32 shows XRD curves of compressed POT as reported by Mårdalen et al. [169]. The data show clearly how both $h 00$ and 010 reflections move towards higher scattering angle as associated with decreasing lamellar period and increasing backbone planarity.

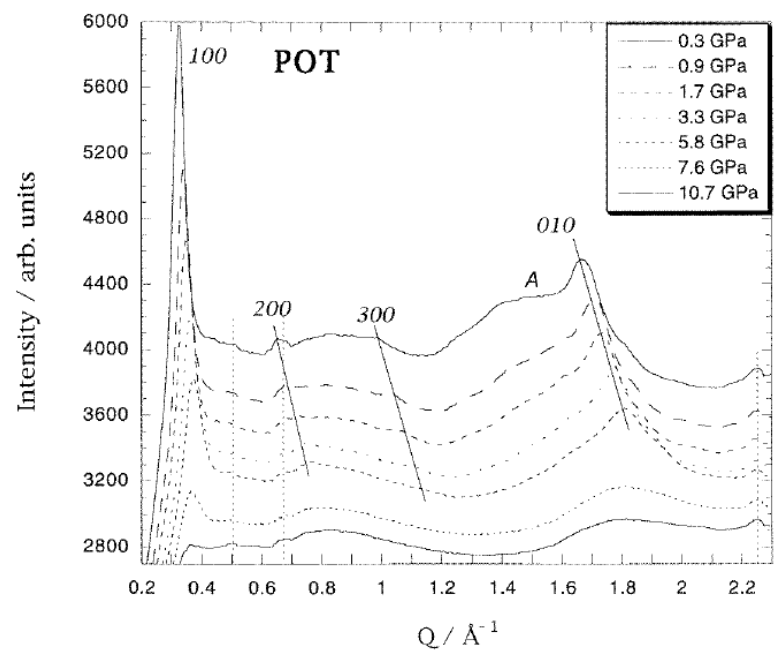

Figure 32. XRD curves of POT polythiophene showing $h 00$ and 010 reflections with increasing pressure. Reproduced from Ref. [169]. Copyright 1998 The Institute of Physics.

More recently, polythiophenes have been widely applied in polymer solar cells especially when blended with methanofullerenes. Particularly well-studied is the blend of poly(3-hexylthiophene) (P3HT) and phenyl-C61-butyric acid methyl ester (PCBM). When blended, they do not mix at the molecular level but remain segregated into polymer and fullerene phases (see, e.g., Ref. [174]). The crystallinity varies depending on the chosen blend ratio and processing conditions.

Paudel et al. studied compressed P3HT-PCBM system first using optical spectroscopy and illustrated decreases in the LUMO and badgap which are associated with the polymer planarization [175]. This was followed by a XRD study of Seki and coworkers who have shown interlamellar P3HT compression and planarization for a P3HT-PCBM blend [176]. The authors also found decreasing crystallite size with increasing pressure presumably related to the increased variation in the $\pi$-stacking distance. Figure 33 shows XRD curves of P3HT:PCBM blend as reported by 
Seki and co-workers [176]. Note that the compression is anisotropic, which typical for $\pi$-conjugated molecules.

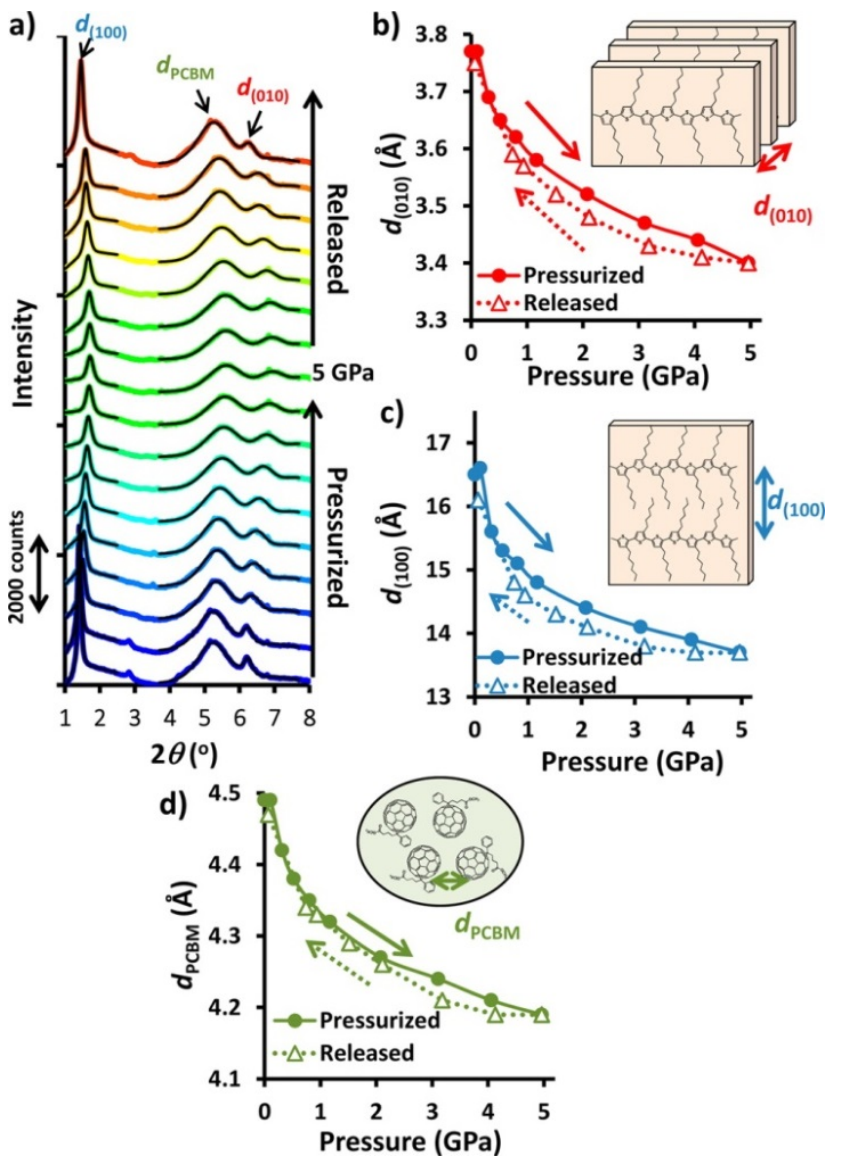

Figure 33. (a) XRD curves of P3HT:PCBM (1:1) blend with increasing and decreasing pressure. The data show 100 and 010 reflections for P3HT and the reflection associated with the intermolecular distance for PCBM. (b-d) The stacking and interlamellar distances for P3HT and intermolecular distance for PCBM as a function of pressure. Reproduced with permission from [176]. Copyright 2015 The American Chemical Society.

\subsubsection{Rigid rod polymers and $\pi$-conjugated rigid rod polymers}

Gitsas et al. [177] studied $\pi$-conjugated poly $(p$ phenylenes) (PPPs) with sulfonated ester and dodecyl side chains under high pressure conditions. At ambient pressure, these polymers show a glass transition and a subsequent liquid crystalline phase that turns to isotropic with heating. When the pressure is increased, both transitions are shifted to higher temperatures. When the pressure is sufficiently high, the isotropic phase is no longer obtained in the attainable temperature range $\left(260-270{ }^{\circ} \mathrm{C}\right)$. Figure 34 shows corresponding phase diagram. Since virtually all hairy-rod type polymers manifest this type of ambient phase behavior, the work of Gitsas et al. provides an important guideline for high pressure studies of other hairy-rod polymers such as PF2/6.

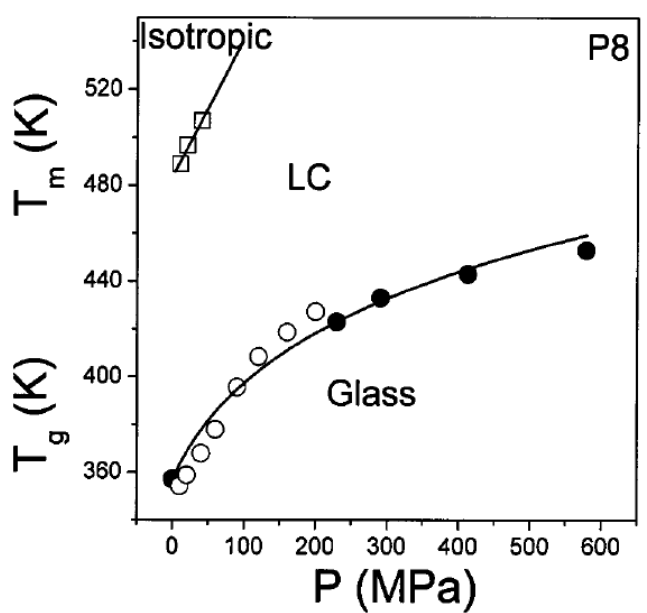

Figure 34. Phase diagram of rigid rod PPP as a function of temperature and pressure. Reproduced with permission from [177]. Copyright 2004 The American Physical Society.

Takizawa et al. [178-181] performed comprehensive studies of rigid rod polyimides. The authors varied the composition of polyimides in terms of phenyl rings in the diamine moiety (and thus effectively the rigidity of the system) and found out that the compressibility along the main chain (c-axis) increases with the increasing number of phenyl rings. Furthermore, depending on this parameter, they were able to identify a system with isotropic or anisotropic compression along interchain directions (a- and b-axes). Figure 35 illustrates this design of pressure-induced anisotropy. This phenomenon, again, is likely to be generalized to other rigid rod polymers and $\pi$-conjugated polymers.

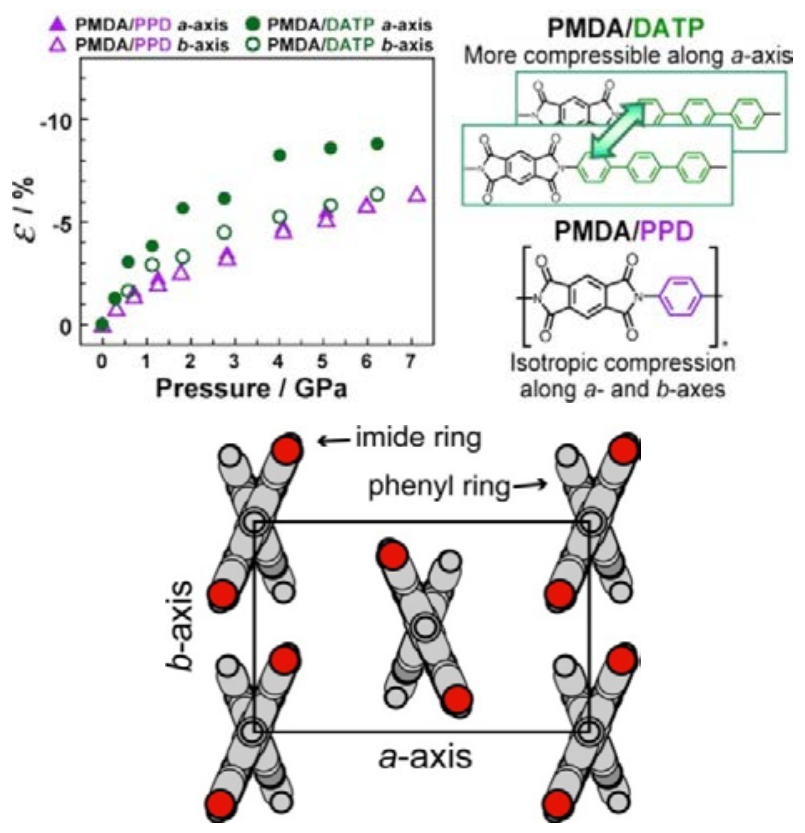

Figure 35. Strain along the crystallographic a- and b-axes for two different polyimides PMDA/DATP and PMDA/PPD with different number of phenyl moieties. The a- and b-axes are parallel and perpendicular to the stacking direction and both perpendicular to the rigid polymer chain that coincidences with the c-axis. Reproduced with permission from [178]. Copyright 2014 The American Chemical Society. 


\subsection{Polyfluorene PF2/6}

\subsubsection{Theoretical considerations}

Earlier studies point to two factors that theoretical considerations should illustrate prior to understanding the high pressure experiments of PFs. First is the impairment of long range order. Whether this means a phase transition to the lower symmetry phase or decreasing crystallite size, pressure may not be able to distinguish the two. Second is the potential backbone planarization as followed by a decrease in the torsion angle $\tau$ between the repeat units.

Pressure driven structural effects on PF2/6 may be approached using packing density arguments as suggested in Ref. [182]. This is based on the previously found first order phase transition between Hex and Nem phases whereby Hex phase turns Nem phase either upon heating or with decreasing molecular weight [43]. However, the earlier consideration assumed constant (and normal atmospheric) pressure. To incorporate pressure to this picture, the shift in the transition temperature as a function of pressure is understood by the Clausius-Clapeyron relation [183]

$$
\frac{d T^{*}}{d P}=\frac{V_{N}-V_{H}}{S_{N}-S_{H}},
$$

where $T^{*}$ denotes the Nem-Hex transition temperature and $V_{i}$ and $S_{i}$ are the volume and entropy of the Nem $(i=N)$ and Hex $(i=H)$ phases.

Following the argumentation of Ref. [182] this was written as

$$
\frac{d T^{*}}{d P}=\frac{A M_{n 0}^{*}}{R T^{*}}\left(\frac{1}{\rho_{N}}-\frac{1}{\rho_{H}}\right),
$$

where $\rho_{i}$ denotes the densities of phases at the transition point and $R$ is the gas constant. $T^{*}$ is the transition temperature for a given pressure and $M_{n 0}^{*}$ the molecular weight $M_{n}$ for the transition at $T_{g}$.

This leads to the transition pressure for the Hex-Nem transition as

$$
P^{*}=P_{r e f}+\frac{R \ln (e / f)}{2 A M_{n 0}} \frac{\rho_{N} \rho_{H}}{\rho_{N}-\rho_{H}}\left(T_{r e f}^{* 2}-T^{2}\right),
$$

where $f$ is the volume fraction of the polymer backbone. This expression and the description above are valid for a nonglassy polymer.

This consideration assumes that PF2/6 is effectively a hairy-rod type polymer with rigid rod backbone and flexible side chains. This is a successful route to understand phase behavior but it does not account the details of changes in the backbone, which is important for pressure studies as pressure is assumed to influence intramolecular structure.

Helical PF2/6 [39, 40, 42] differs from more planar PF8 that manifest a repeat distance of four monomers for the crystalline $\alpha$ phase [184] or a repeat distance of two monomers for the mesomorphic $\beta$ phase [185]. Assuming PF2/6 planarization with increasing pressure, a suitable model should incorporate trans or near trans torsion angles and allow at least 4-fold and 2-fold periodicities.

Figure 36 illustrates different models for the $\mathrm{PF}$ backbone conformations as described in Refs. [186, 187]. These manifest different symmetries about the c-axis and produce the $00 \mathrm{l}$ reflections which can be followed by XRD experiments. The first two geometries represent regular $4 / 1$ and 2/1 helixes. The third geometry (TS) ${ }_{2}$ has alternating trans and near-trans torsion angles. The fourth geometry $(\mathrm{GS})_{2}$ contains two rotations that put the third monomer in the trans position to the first. The two rotations have one degree of freedom and satisfy $\tau_{\mathrm{s}}+\tau_{\mathrm{g}} \sim 180^{\circ}$. The two last geometries manifest twisted conformations through alternating left-hand right-hand rotations $\tau \mathrm{s}$ and $-\tau \mathrm{s}$.

Figure 37 plots the monomer periods corresponding to 001 reflections for helical structures and for the (GS)2 and (TS)2 geometries as described in Ref. [186]. The bond distances are based on dioctylfluorene after McFarlane et al. [188]. The monomer repeat depends on $\tau$ and on the bond angle between fluorene repeats. For the assumed fixed bond angle of $11.43^{\circ}$, the $21 / 4$ helix is realised with a torsion angle 65.09 o, for example. This corresponds to the repeat unit 8.06 $\AA$ which is in good agreement with the experimental data [42]. Any pressure driven planarization of PF2/6 should lead to a decreased monomer period and be seen as a shift of 001 reflections towards higher scattering angles.

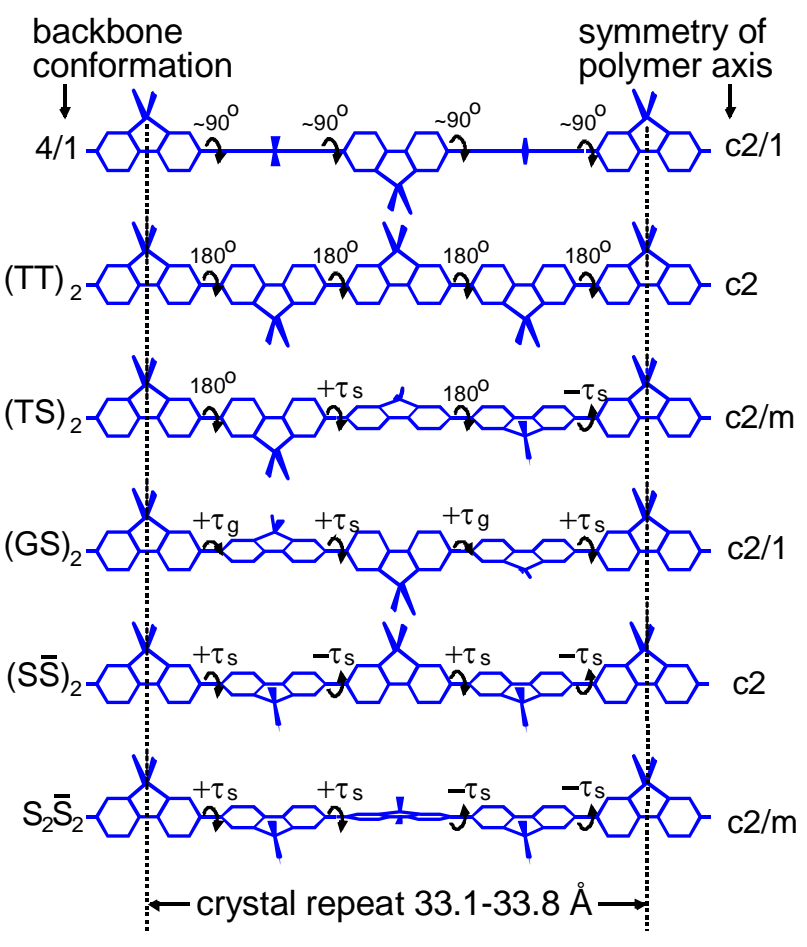

Figure 36. Models for the polyfluorene backbone conformations that manifest different symmetries about the c-axis and produce the $00 \mathrm{l}$ reflections. The models are characterized by torsion angles $\sim 90^{\circ}$, $180^{\circ}, \pm \tau_{\mathrm{s}}$, and $\pm \tau_{\mathrm{g}} . \mathrm{T}=$ trans. $\mathrm{S}=$ skew. $\mathrm{G}=$ gauche. Reproduced with permission from [186]. Copyright 2013 The American Physical Society. 


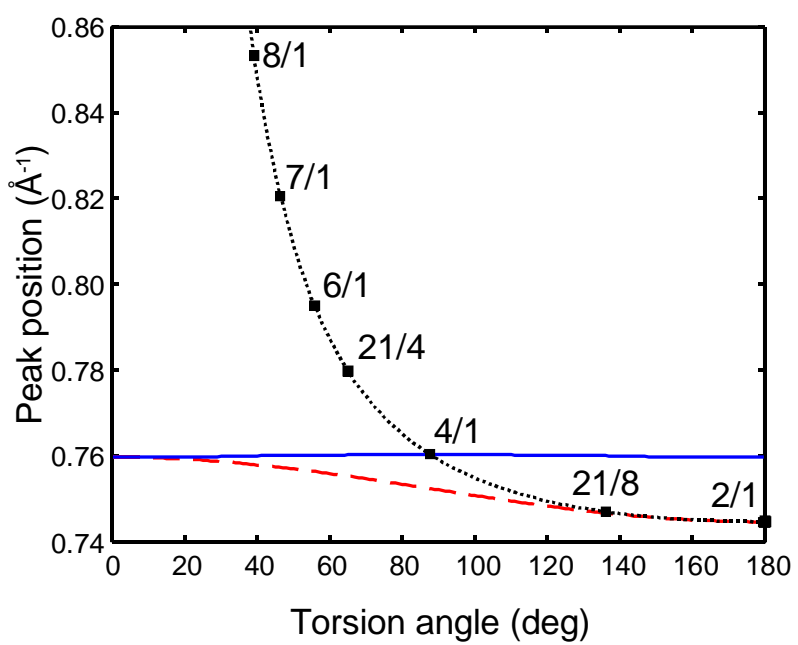

Figure 37. Theoretically predicted position of the most prominent $00 \mathrm{l}$ $\mathrm{X}$-ray reflection as a function of torsion angle for various helical (dotted black line and square markers) and non-helical structures where the base unit consist of two (solid blue line - $(\mathrm{GS})_{2}$ ) or four alternating torsion angles (dashed red line- $(\mathrm{TS})_{2}$ ). Reproduced with permission from [186]. Copyright 2013 The American Physical Society.

\subsubsection{Pressure induced structural effects}

Figure 38 (a) shows the XRD data of PF2/6 with increasing pressure as reported in Ref. [186]. These data show a peak at $0.82 \AA^{-1}$ and a shoulder at $0.86 \AA^{-1}$ which are indexed as $00 l$ and $h 00$, and fitted to a two peak model. At ambient conditions these reflections correspond to 0021 and 200. Figure 38 (b) plots the peak positions deduced from these data.

The shift of $h 00$ peak together with the broadening of $00 l$ implies impairment of the ambient hexagonal order which begins at or below $2 \mathrm{GPa}$. This change seems similar as the ambient Hex-Nem transition (as discussed in the section above and in Ref. [182]) and is concomitant with sharp optical changes shown in Ref. [189]. However, the nature of transition is not the same. The ambient transition occurs above the glass transition but the pressure induced loss of hexagonal structure occurs below the glass transition. Therefore, we denote the obtained structure as glassy nematic phase. We have previously calculated that ambient LMW Nem state is denser than Hex phase $\left(1.1 \mathrm{~g} / \mathrm{cm}^{3}\right.$ vs $\left.0.9 \mathrm{~g} / \mathrm{cm}^{3}\right)$ [43]. It is not possible to determine the full set of lattice parameters from the high pressure data. However, the direction of the density change is plausibly towards the denser phase.

Figure 39 shows similar XRD data and Figure 40 shows the 001 reflection deduced from these data and interpreted against the theoretically calculated model helices with decreasing torsion angle $\tau$ (see the section above). This consideration indicates that the PF2/6 backbone is planarized ( $\tau$ decreased) with increasing pressure. This result is further supported by the minimum energy conformation with the torsion angle at $43^{\circ}$ as calculated from the first principles by Tanto et al.[40].

At ambient conditions, LMW PF2/6 and HMW PF2/6 Copyright 2013 The American Physical Society. have significant difference in the long range order (Figure 3). Furthermore, a slight deviation in the $00 \mathrm{l}$ reflections towards higher scattering angle indicates that the torsion angle and thus the intramolecular structure of ambient LMW PF2/6 is not identical to HMW PF2/6 [43]. Figure 41 shows the most prominent $00 l$ peak in LMW PF2/6 with increasing pressure as reported in Ref. [44]. The peak is shifted towards higher scattering angles indicating polymer planarization consistent with the high pressure Raman data shown in Section 5.3. However, the peak width stays constant, which indicates that its intermolecular structure is maintained on compression.
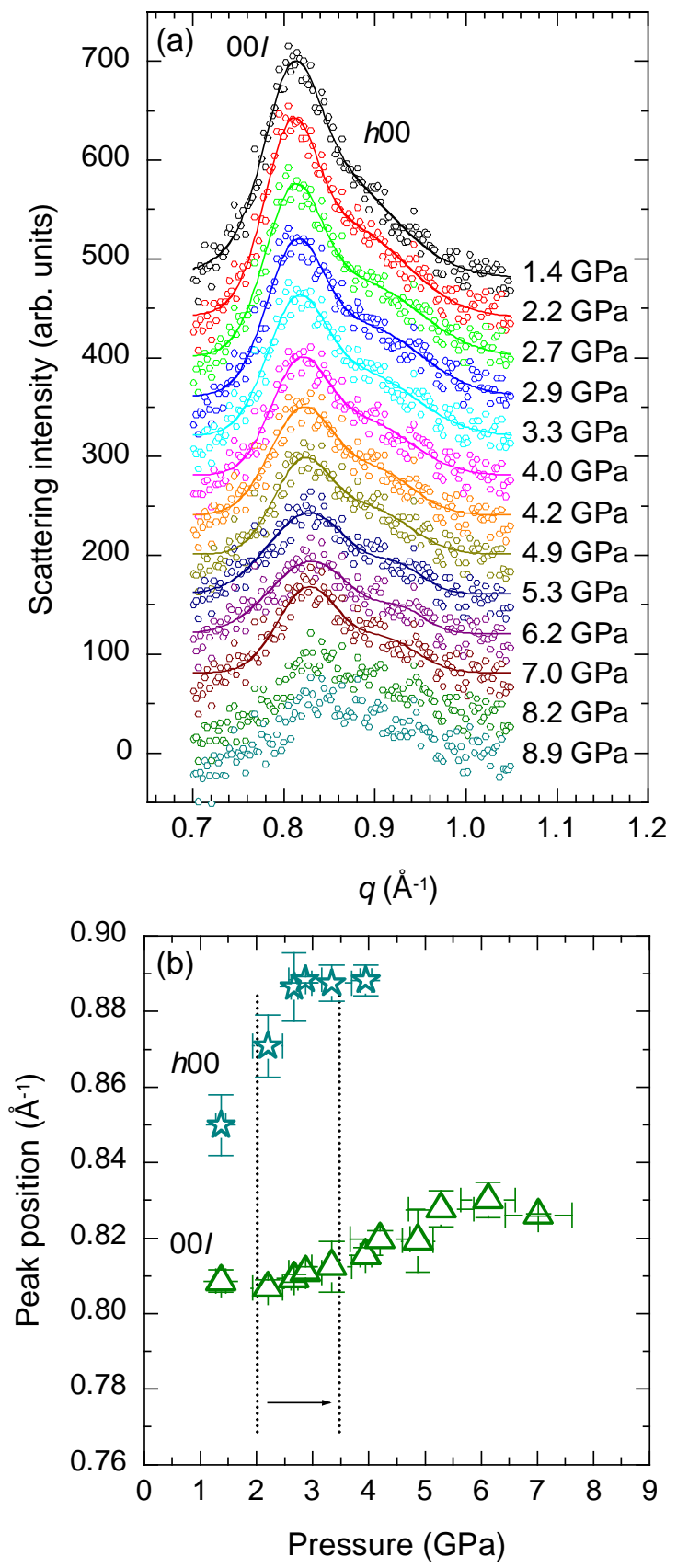

Figure 38. (a) XRD data of PF2/6 during compression. Solid lines represent a two-peak scattering model with the assumed $00 l$ and $h 00$ peaks. (b) Peak positions deduced from this model. Dashed vertical lines mark the area corresponding to the sharp optical changes as reported in Ref. [189]. Reproduced with permission from [186]. 


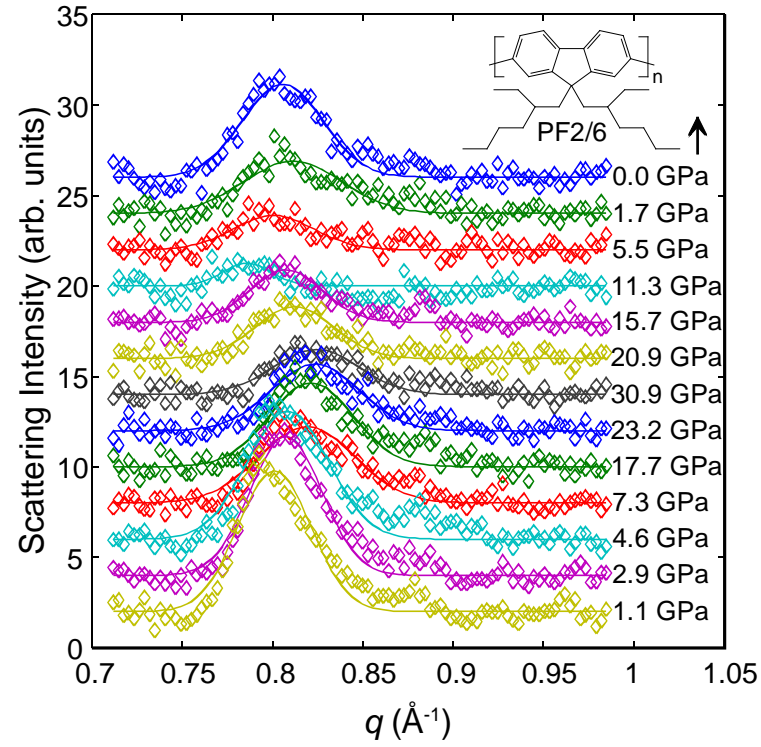

Figure 39. XRD curves (diamonds) and fits to the data (solid lines) of PF2/6 during compression and decompressions. Reproduced with permission from [187]. Copyright 2013 The American Chemical Society.

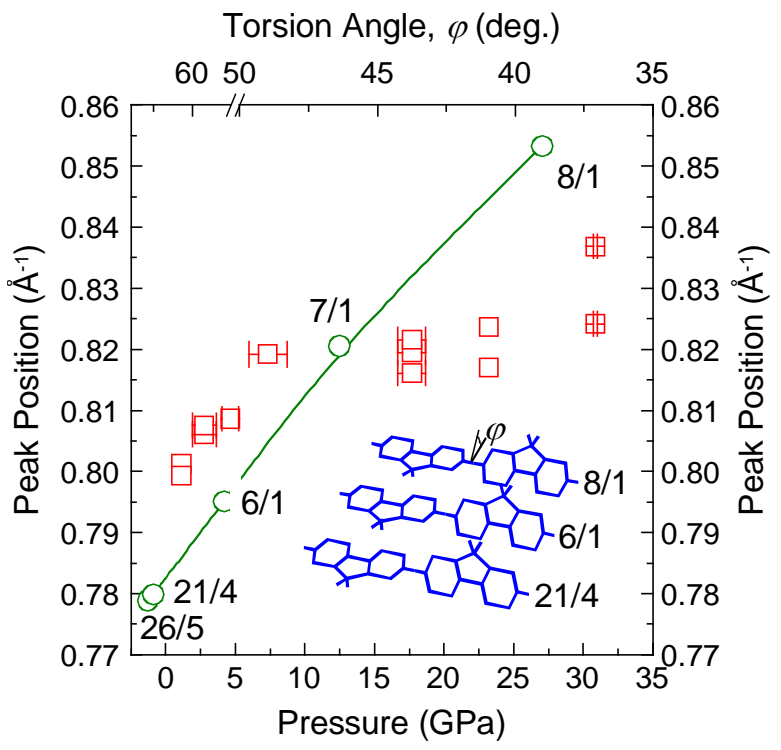

Figure 40. Position of the strongest 001 reflection against pressure (red squares) as a function of pressure corresponding to the data shown in Figure 39. The peak position for selected theoretical PF2/6 helixes (open circles) as a function of torsion angle that is this reference denoted as $\varphi$.. Reproduced with permission from [187]. Copyright 2013 The American Chemical Society.

$$
q\left(\AA^{-1}\right)
$$
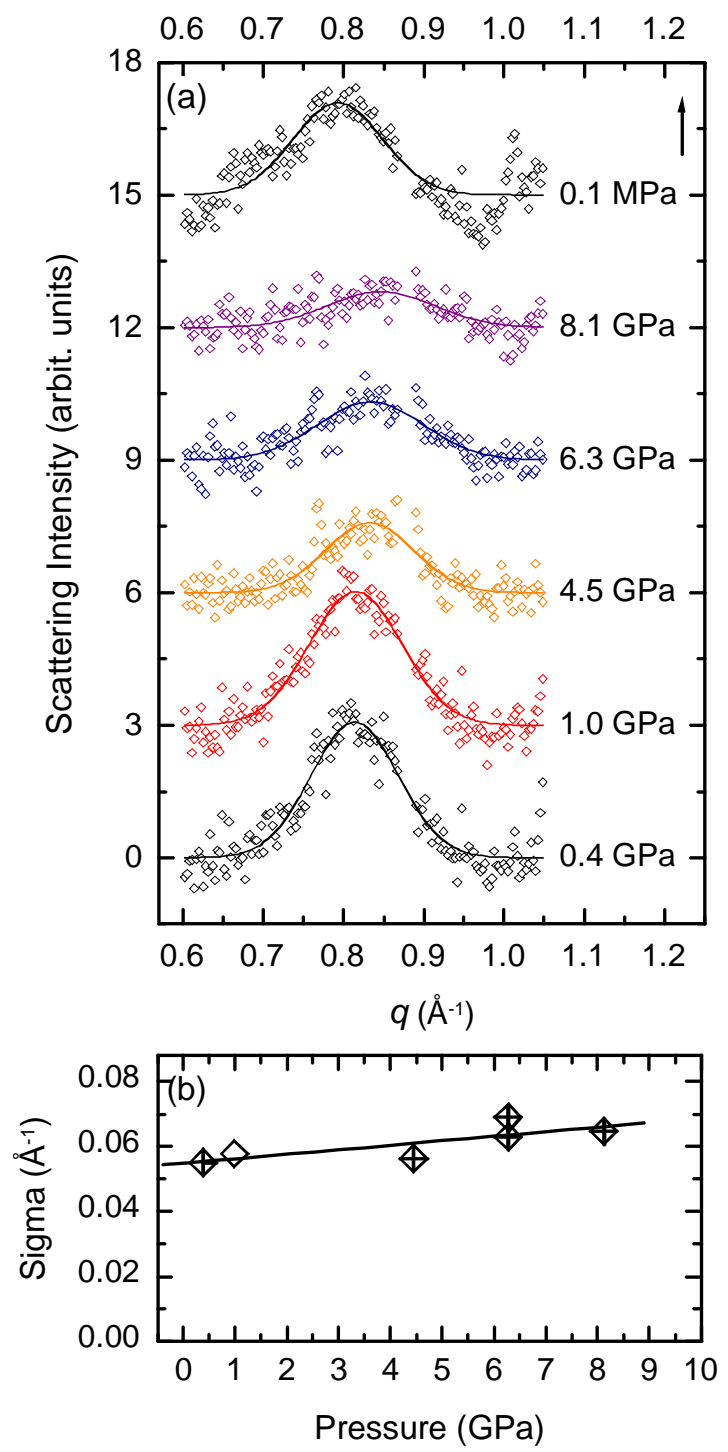

Figure 41. (a) XRD patterns of LMW PF2/6 at selected pressures and fits of the most prominent $00 l$ peak. (b) Peak width of the same data. Reproduced with permission from [44]. Copyright 2014 Wiley Periodicals, Inc.

\subsubsection{Structural inhomogeneity under pressure}

Many pressure transmitting media solidify and their hydrostatic limits are well below the pressure maxima of current DACs as described by Klotz et al. [190]. For example, commonly used methanol-ethanol mixture becomes non-hydrostatic around $10 \mathrm{GPa}$ and neon at $15 \mathrm{GPa}$. When the system is non-hydrostatic, making a distinction between the inhomogeneous pressure distribution and the effect of pressure becomes difficult. This has been a reason why maximum pressures used for $\pi$-conjugated polymers have been 7-8 GPa. Pressures beyond this limit would, however, allow us to find phases and phenomena not existing at lower pressures. Scaling of polymer properties with pressure would become more accurate and lead to more quantitative data analysis if XRD studies are conducted at higher pressures.

We have proposed that the pressure inhomogeneity could 
be handled by mapping the sample by a microbeam and comparing the obtained data distribution to the pressure distribution obtained from the analysis of pressure standards. Figure 42 shows maps of the strongest $00 \mathrm{l}$ reflection and the crystallite orientation for compressed PF2/6 as reported in Ref. [187] . Each point corresponds to the dataset like the one shown in Figure 39 and are reduced to plot the maps shown in Figure 40. The photos of the sample in the gasket corresponding to the maps are shown on the background. These data show a sharp widening in the distribution of the pressure induced effect in structure above 7-8 GPa, which justifies the mapping in above these limits. In contrast, the crystallite orientation distribution is not significantly influenced by pressure.

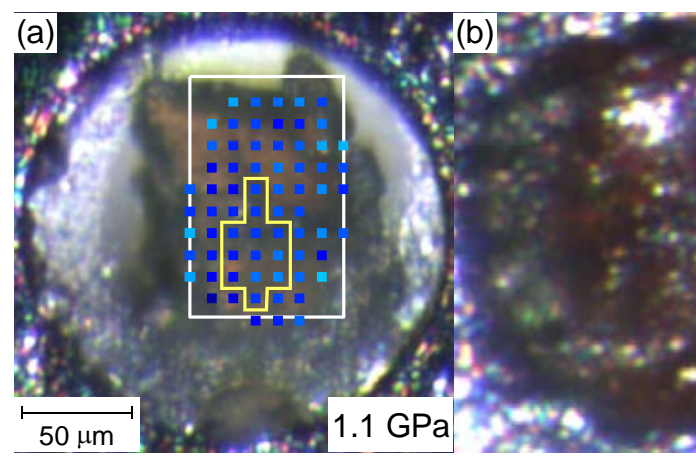

(d)

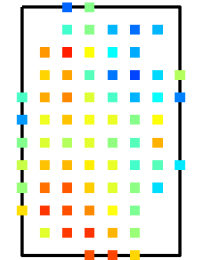

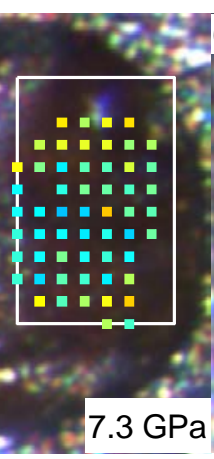

(e)

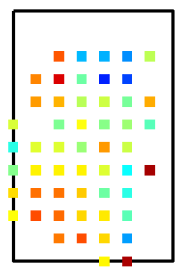

(f)
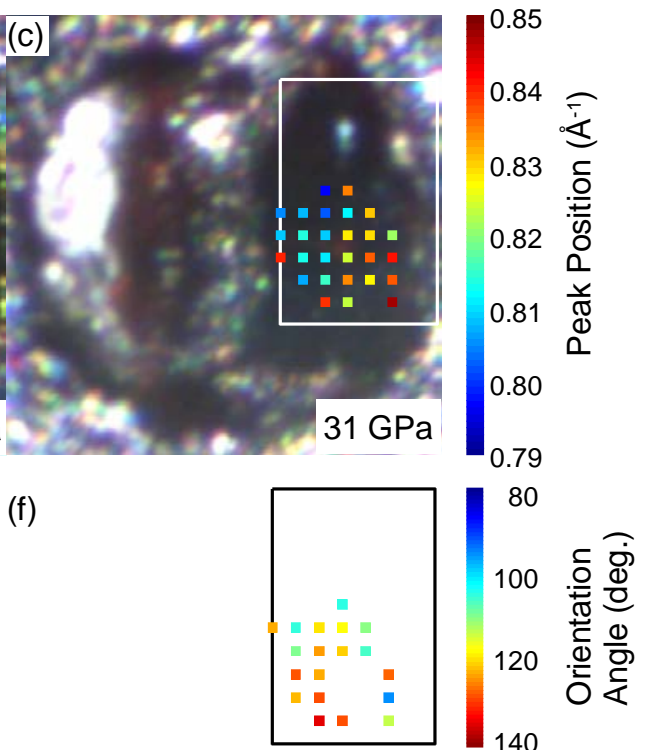

Figure 42. (a-c) Photos of the compressed samples within the DAC gasket at various pressures and maps of the peak positions for the strongest $00 \mathrm{l}$ reflection. (d-f) Corresponding maps for the preferred orientation direction. Yellow polygon marks the area corresponding to the data shown in Figure 31. Reproduced with permission from [187]. Copyright 2013 The American Chemical Society.

\section{Summary and outlook}

$\pi$-Conjugated polymers and organic semiconductors in general manifest complex electrical, optical and structural behaviour. This review illustrates pressure induced effects on their electronic and vibrational states as well as phase transitions, molecule planarization and local and macroscopic anisotropy. Beginning from archetypical blue emitting systems - oligophenylenes and polyfluorenes - these effects have been discussed from experimental and theoretical point of view for a few systems.

In many occasions, the current literature provides solid evidence and guidelines for pressure induced effects on intraand intermolecular structure and its electronic and optical realizations. Yet the current literature is not comprehensive in terms of what is achievable with high pressure. The details of thermodynamic vs kinetic factors behind polymorphism are largely unknown. The effects of pressure may depend on the processing history including compression rate and direction and whether oriented or powder samples are considered. This all requires systematic work from different families of conjugated polymers under pressure.

Due to the high resistivity of conjugated polymers and challenges in attaching electrical contacts in a DAC with soft materials, concomitant electrical measurements with electronic spectra under high pressure hardly exist. Only recently it was demonstrated that by using an ionic liquid both as the gate dielectric in a thin film polymer transistor as well as the pressure medium, a cross-over from a 1D hopping to 3D hopping transport occurs under pressure [191]. Such studies are essential for obtaining a complete picture of the impact of the pressure on the charge transport properties of $\pi$ conjugated molecules and polymers.

In years to come, we expect high pressure studies becoming more and more common in the fields of $\pi$ conjugated molecules and foresee several advances that stem from new modelling, materials synthesis, and instrumentation. So far the studies have focused on relatively simple model materials and phase transitions or compression of the ambient phase. Future studies are likely to move towards more complicated materials and towards high pressure synthesis as already shown for -N(NO)N(NO) polymers analogous to cis-polyacetylene [192]. With this vision, it is feasible that chemical transformations are induced by pressure and temperature or light induced transformations under pressure result in new compounds, which are not achievable through ambient routes.

Molecular spintronics, which combines the potential of spintronics and organic electronics is a promising alternative to conventional spintronics with inorganic materials [193]. The mixing of the triplet and singlet states as a result of the hyperfine field (of the hydrogen atoms) in organic semiconductors manifests itself as a large magnetoresistance effect [194, 195]. Presumably, if electrical leads could be easily attached to conjugated polymers and molecules in a DAC for magneto-electric measurements, high pressure could serve as a powerful tuning parameter to study spin transport 
in organic spintronics.

Furthermore, we expect that this field will benefit from new sophisticated X-ray instrumentation. Already existing submicrometer level beam size approaches polymer crystallite size and will allow the sample mapping in the level of individual crystallites. Novel structures may be discovered by new techniques involving $\mathrm{x}$-ray lasers, which are already being harnessed to connect time-resolved optical processes with structural properties of materials. Similarly, powerful neutron sources allowing significantly smaller sample cells, and consequently higher pressures so far being possible only with X-ray studies, may yield new insights into both magnetic and non-magnetic organic semiconductors

\section{Aknowledgements}

We would like to thank several of our collaborators who have played a major role in the development of the field; without their important contribution this review would have never come to fruition. Meera Chandrasekhar and Willi Graupner laid the foundation for optical spectroscopy of conjugated polymers under high pressure. Ulli Scherf not only provided most of the conjugated polymers presented in this work but has throughout guided us in understanding the structure-property relationship in these systems. We have greatly benefitted from our interaction with Michael Winokur, who has given deep insights into the structural properties of PFs. We thank S Ramasesha and Roman Stepanyan for theoretical insights on the impact of pressure on the electronic properties and structure formation.

Chris Martin, Keshab Paudel, and Mohammad Arif were instrumental in undertaking several optical measurements of PFs and analysis under pressure. Chris Volz and Soma Khanra are acknowledged for the DFT calculations (including vibrational spectra) and Mika Torkkeli, Dörthe Haase, Stefan Carlson, Zuzana Konopkôvá, and Hanns-Peter Liermann for work with X-ray scattering. 


\section{References}

[1] Bridgman P W 1935 Theoretically interesting aspects of high pressure phenomena Rev. Mod. Phys. 71

[2] Bridgman P W 1945 Effects of high hydrostatic pressure on the plastic properties of metals Rev. Mod. Phys. 173

[3] Bridgman P W 1946 Recent work in the field of high pressures: Addendum Rev. Mod. Phys. 18291

[4] Drickamer H G (1965) Solid-state physics vol 17: Academic, New York), pp 1

[5] Drickamer H G (1966) Solid-state physics vol 19: Academic, New York), pp 135

[6] Merrill L and Bassett W A 1974 Miniature diamond anvil pressure cell for single crystal x-ray diffraction studies Rev. Sci. Instrum. 45290

[7] Paul W 1961 Band structure of intermetallic semiconductors from pressure experiments J. Appl. Phys. 32 Supplement 2082

[8] Cardona M, Paul W and Brooks H 1959 Dielectric constant of germanium and silicon as a function of volume J. Phys. Chem. Solids 8204

[9] Dacorogna M M, Chang K J and Cohen M L 1985 Pressure increase of the electron-phonon interaction in superconducting hexagonal silicon Phys. Rev. B 321853

[10] Erskine D, Yu P Y, Chang K J and Cohen M L 1986 Superconductivity and phase transitions in compressed si to 45 gpa Phys. Rev. Lett. 572741

[11] Chu C W, Gao L, Chen F, Huang Z J, Meng R L and Xue $\mathrm{Y} Y 1993$ Superconductivity above $150 \mathrm{k}$ in hgba2ca2cu3o8 $+\delta$ at high pressures Nature 365323

[12] Dubrovinsky L, Dubrovinskaia N, Prakapenka V B and Abakumov A M 2012 Implementation of micro-ball nanodiamond anvils for high-pressure studies above 6 mbar Nat. Commun. 31163

[13] Mao H-k and Hemley R J 1994 Ultrahigh-pressure transitions in solid hydrogen Rev. Mod. Phys. 66671

[14] Dzyabura V, Zaghoo M and Silvera I F 2013 Evidence of a liquid-liquid phase transition in hot dense hydrogen Proc. Natl. Acad. Sci. 1108040

[15] Jeanloz R, Celliers P M, Collins G W, Eggert J H, Lee K K M, McWilliams R S, Brygoo S and Loubeyre P 2007 Achieving high-density states through shock-wave loading of precompressed samples Proc. Natl. Acad. Sci. 1049172

[16] Ma J, Fischer J E, Cao Y and Heeger A J 1992 X-ray structural study of trans-polyacetylene at high pressure Solid State Commun. 83395

[17] Menon R, Väkiparta K, Cao Y and Moses D 1994 Pressure dependence of the conductivity and magnetoconductance in oriented iodine-doped polyacetylene Phys. Rev. B 4916162

[18] Väkiparta K, M R, Andersson M, Cao Y, Moses D and Heeger A 1993 Temperature dependence of the electrical conductivity of potassium-doped polyacetylene as a function of pressure and magnetic field Phys. Rev. B 47 9977

[19] Meersam F and McMillan P F 2014 High hydrostatic pressure: A probing tool and a necessary parameter in biophysical chemistry Chem. Commun 50766

[20] Rogers J A, Bao Z, Baldwin K, Dodabalapur A, Crone B, Raju V R, Kuck V, Katz H, Amundson K, Ewing J and
Drzaic P 2001 Paper-like electronic displays: Large-area rubber-stamped plastic sheets of electronics and microencapsulated electrophoretic inks Proc. Natl. Acad. Sci. 984835

Heeger A J 2001 Nobel lecture: Semiconducting and metallic polymers: The fourth generation of polymeric materials Rev. Mod. Phys. 73681

Burroughes J H, Bradley D D C, Brown A R, Marks R N, Mackay K, Friend R H, Burns P L and Holmes A B 1990 Light-emitting diodes based on conjugated polymers Nature 347539

Kalinowski J (2004) Organic light-emitting diodes: Principles, characteristics \& processes (optical science and engineering) (New York: Marcel Dekker)

Zaumseil J and Sirringhaus H 2007 Electron and ambipolar transport in organic field-effect transistors Chem. Rev. 107 1296

[25] Allard S, Forster M, Souharce B, Thiem H and Scherf U 2008 Organic semiconductors for solution-processable field-effect transistors (ofets) Angew. Chem. Int. Ed. 47 4070

[26] Schwartz G, Tee B C K, Mei J, Appleton A L, Kim D H, Wang $\mathrm{H}$ and Bao Z 2013 Flexible polymer transistors with high pressure sensitivity for application in electronic skin and health monitoring Nat. Commun. 41859

Brabec C J, Dyakonov V, Parisi J and Sariciftci N S (2003) Organic photovoltaics: Concepts and realization (Berlin: Springer Verlag)

[28] Peet J, Kim J Y, Coates N E, Ma W L, Moses D, Heeger A $\mathrm{J}$ and Bazan G C 2007 Efficiency enhancement in lowbandgap polymer solar cells by processing with alkane dithiols Nat. Mater. 6497

Martens H C F, Blom P W M and Schoo H F M 2000 Comparative study of hole transport in poly( p-phenylene vinylene) derivatives Phys. Rev. B 617489

[30] Kemerink M, van Duren J K J, Jonkheijm P, Pasveer W F, Koenraad P M, Janssen R A J, Salemink H W M and Wolter J H 2003 Relating substitution to single-chain conformation and aggregation in poly(p-phenylene vinylene) films Nano Lett. 31191

[31] McCullough R D 1998 The chemistry of conducting polythiophenes Adv. Mater. 1093

[32] Baker K N, Fratini A V, Resch T, Knachel H C, Adams W W, Socci E P and Farmer B L 1993 Crystal structures, phase transitions and energy calculations of poly(pphenylene) oligomers Polymer 341571

Guha S, Graupner W, Resel R, Chandrasekhar M, Chandrasekhar H R, Glaser R and Leising G 2001 Tuning intermolecular interactions: A study of the structural and vibrational properties of p-hexaphenyl under pressure $J$. Phys. Chem. A 1056203

[34] Socci E P, Farmer B L and Adams W W 1993 Molecular dynamics simulations of a poly(p-phenylene) oligomer $J$. Polym. Sci. Part B Polym. Phys. 311975

Scherf U 1999 Ladder-type materials J. Mater. Chem. 9 1853

Scherf U and Müllen K 1991 Polyarylenes and poly(arylenevinylenes), 7. A soluble ladder polymer via bridging of functionalized poly(p-phenylene)-precursors Macrom. Rapid Comm. 12489

Tasch S, List E J W, Ekström O, Graupner W, Leising G, 
Schlichting P, Rohr U, Geerts Y, Scherf U and Müllen K 1997 Efficient white light-emitting diodes realized with new processable blends of conjugated polymers Appl. Phys. Lett. 712883

[38] Stagira S, Zavelani-Rossi M, Nisoli M, DeSilvestri S, Lanzani G, Zenz C, Mataloni P and Leising G 1998 Singlemode picosecond blue laser emission from a solid conjugated polymer Appl. Phys. Lett. 732860

[39] Lieser G, Oda M, Miteva T, Meisel A, Nothofer H-G, Scherf U and Neher D 2000 Ordering, graphoepitaxial orientation, and conformation of a polyfluorene derivative of the "Hairy-rod" Type on an oriented substrate of polyimide Macromolecules 334490

[40] Tanto B, Guha S, Martin C M, Scherf U and Winokur M J 2004 Structural and spectroscopic investigations of bulk poly[bis(2-ethyl)hexylfluorene] Macromolecules 379438

[41] Brinkmann M, Charoenthai N, Traiphol R, Piyakulawat P, Wlosnewski J and Asawapirom U 2009 Structure and morphology in highly oriented films of poly(9,9-bis(noctyl)fluorene-2,7-diyl) and poly(9,9-bis(2ethylhexyl)fluorene-2,7-diyl) grown on friction transferred poly(tetrafluoroethylene) Macromolecules 428298

[42] Knaapila M, Torkkeli M and Monkman A P 2007 Evidence for 21-helicity of poly[9,9-bis(2-ethylhexyl)fluorene-2,7diyl] Macromolecules $\mathbf{4 0} 3610$

[43] Knaapila M, Stepanyan R, Torkkeli M, Lyons B P, Ikonen T P, Almásy L, Foreman J P, Serimaa R, Güntner R, Scherf $\mathrm{U}$ and Monkman A P 2005 Influence of molecular weight on the phase behavior and structure formation of branched side chain hairy-rod polyfluorene in bulk phase Phys. Rev. E 71041802

[44] Guha S, Knaapila M, Moghe D, Konôpková Z, Torkkeli M, Fritsch M and Scherf U 2014 Persistence of nematic liquid crystalline phase in a polyfluorene-based organic semiconductor: A high pressure study J. Polym. Sci. Part B Polym. Phys. 521014

[45] Chen S H, Chou H L, Su A C and Chen S A 2004 Molecular packing in crystalline poly(9,9-di-n-octyl-2,7fluorene) Macromolecules 376833

[46] Winokur M J, Slinker J and Huber D L 2003 Structure, photophysics, and the order-disorder transition to the beta phase in poly(9,9-(di-n,n-octyl)fluorene) Phys. Rev. B 67 184106

[47] Ariu M, Lidzey D G, Sims M, Cadby A J, Lane P A and Bradley D D C 2002 The effect of morphology on the temperature-dependent photoluminescence quantum efficiency of the conjugated polymer $\operatorname{poly}(9$, 9dioctylfluorene) J. Phys.: Condens. Matter 149975

[48] Chunwaschirasiri W, Tanto B, Huber D L and Winokur M J 2005 Chain conformations and photoluminescence of poly(di-\$n\$-octylfluorene) Phys. Rev. Lett. 94107402

[49] Guha S 2008 Raman spectroscopic studies of polyfluorenes The Open Physical Chemistry Journal 26

[50] Bruno M S and Dunn K J 1984 Stress analysis of a beveled diamond anvil Rev. Sci. Instrum. 55940

[51] Venkateswaran U and Chandrasekhar M 1985 Lowtemperature studies of the photoluminescence in cds under hydrostatic pressure Phys. Rev. B 311219

[52] Venkateswaran U, Chandrasekhar M, Chandrasekhar H R, Wolfram T, Fischer R, Masselink W T and Morkoç H 1985 Photoluminescence studies of a gaas-ga1-x alxas superlattice at $8-300 \mathrm{k}$ under hydrostatic pressure (0-70 kbar) Phys. Rev. B 314106
Piermarini G J, Block S, Barnett J D and Forman R A 1975 Calibration of the pressure dependence of the r1 ruby fluorescence line to $195 \mathrm{kbar}$ J. Appl. Phys. 462774

[54] Mao H K, Bell P M, Shaner J W and Steinberg D J 1978 Specific volume measurements of $\mathrm{cu}, \mathrm{mo}$, pd, and ag and calibration of the ruby r1 fluorescence pressure gauge from 0.06 to 1 mbar J. Appl. Phys. 493276

Wang Z, He D, Zhang W, Li W, Li W, Qin J, Lei L, Zou Y and Yang X 2010 Portable high pressure sapphire anvil cell for gas hydrates research Rev. Sci. Instrum. 81085102

[56] Katrusiak A 2008 High-pressure crystallography Acta Cryst. A 64135

Boldyreva E V 2007 High-pressure diffraction studies of molecular organic solids. A personal view Acta Cryst. A 64 218

[58] Sahle C J, Sternemann C, Schmidt C, Lehtola S, Jahn S, Simonelli L, Huotari S, Hakala M, Pylkkänen T, Nyrow A, Mende K, Tolan M, Hämäläinen K and Wilke M 2012 Microscopic structure of water at elevated pressures and temperatures Proc. Natl. Acad. Sci. 1106301

Chiba A, Funamori N, Nakayama K, Ohishi Y, Bennington S M, Rastogi S, Shukla A, Tsuji K and Takenaka M 2012 Pressure-induced structural change of intermediate-range order in poly(4-methyl-1-pentane) melt Phys. Rev. E 85 021807

[60] Leute U and Dollhopf W 1980 A review of the experimental-data from the high pressure phase in polyethylene Colloid Polym. Sci. 258353

[61] Eby R K, Clark E S, Farmer B L, Piermarini G J and Block S 1990 Crystal structure of poly(tetrafluoroethylene) homoand copolymer in the high pressure phase Polymer 312227

[62] Lorenzen M, Hanfland M and Mermet A 2003 Poly(tetrafluoroehtylene) under pressure: X-ray diffraction studies Nucl. Instr. and Meth. in Phys. Res. B 200416

[63] Rae P J and Dattelbaum D M 2004 The properties of poly(tetrafluoroethylene) (ptfe) in compression Polymer 45 7615

[64] Soignard E, Benmore C J and Yarger J L 2010 A perforated diamond anvil cell for high-energy x-ray diffraction of liquids and amorphous solids at high pressure Rev. Sci. Instrum. 81035110

Pope M and Swenberg C E (1999) Electronic processes in organic crystals and polymers, 2nd edition (New York: Oxford University Press)

[66] Barford W (2009) Electronic and optical properties of conjugated polymers (New York: Oxford University Press)

Bässler H and Schweitzer B 1998 Site-selective fluorescence spectroscopy of conjugated polymers and oligomers Acc. Chem. Res. 32173

Friend R H, Bradley D D C and Townsend P D 1987 Photo-excitation in conjugated polymers J. Phys. D: Appl. Phys. 201367

Kersting R, Lemmer U, Mahrt R F, Leo K, Kurz H, Bässler $\mathrm{H}$ and Göbel E O 1993 Femtosecond energy relaxation in pi-conjugated polymers Phys. Rev. Lett. 703820

Drickamer H G and Frank C W (1973) Electronic transistions and the high pressure chemistry and physics of solids (New York: Chapman and Hall, Ltd.)

Drickamer H G, Frank C W and Slichter C P 1972 Optical versus thermal transitions in solids at high pressure Proc. Natl. Acad. Sci. 69933 
configuration coordinate parameters from high pressure [89] optical data. I. Phenanthrene, anthracene, and tetracene $J$. Chem. Phys. 612870

[73] Tasch S, Niko A, Leising G and Scherf U 1996 Highly efficient electroluminescence of new wide band gap laddertype poly(para-phenylenes) Appl. Phys. Lett. 681090

[74] Yang Y, Pei Q and Heeger A J 1996 Efficient blue polymer light-emitting diodes from a series of soluble poly(paraphenylene)s J. Appl. Phys. 79934

[75] Yoshino K, Nakao K, Onoda M and Sugimoto R 1989 Photoluminescence of poly(3-alkylthiophene) under hydrostatic pressure J. Phys.: Condens. Matter 11009

[76] Hess B, Kanner G and Vardeny Z 1993 Photoexcitations in polythiophene at high pressure Phys. Rev. B 471407

[77] Webster S and Batchelder D N 1996 Absorption, luminescence and raman spectroscopy of poly(p-phenylene vinylene) at high pressure Polymer 374961

[78] Yang G, Li Y, White J O and Drickamer H G 1999 Effect of pressure on the fluorescence of poly[2-methoxy-5-(2'ethylhexoxy)-p-phenylenevinylene] The Journal of Physical Chemistry B 1035181

[79] Guha S, Rice J D, Yau Y T, Martin C M, Chandrasekhar M, Chandrasekhar H R, Guentner R, Scanduicci de Freitas $P$ and Scherf U 2003 Temperature-dependent photoluminescence of organic semiconductors with varying backbone conformation Phys. Rev. B 67125204

[80] Guha S and Chandrasekhar M 2004 Photophysics of organic emissive semiconductors under hydrostatic pressure Phys. Status Solidi B 2413318

[81] Guha S, Graupner W, Yang S, Chandrasekhar M, Chandrasekhar H R and Leising G 1999 Optical properties of poly(para-phenylenes) under high pressure Phys. Status Solidi B 211177

[82] Liu Z X, Goñi A R, Syassen K, Siegle H, Thomsen C, Schöttker B, As D J and Schikora D 1999 Pressure and temperature effects on optical transitions in cubic gan $J$. Appl. Phys. 86929

[83] Ves S, Venkateswaran U D, Loa I, Syassen K, Shahedipour F and Wessels B W 2000 Pressure dependence of the blue luminescence in mg-doped gan Appl. Phys. Lett. 772536

[84] Guha S, Cai Q, Chandrasekhar M, Chandrasekhar H R, Kim H, Alvarenga A D, Vogelgesang R, Ramdas A K and Melloch M R 1998 Photoluminescence of short-period gaas/alas superlattices: A hydrostatic pressure and temperature study Phys. Rev. B 587222

[85] Chelikowsky J R and Cohen M L 1976 Nonlocal pseudopotential calculations for the electronic structure of eleven diamond and zinc-blende semiconductors Phys. Rev. B 14556

[86] Puschnig P, Hummer K, Ambrosch-Draxl C, Heimel G, Oehzelt M and Resel R 2003 Electronic, optical, and structural properties of oligophenylene molecular crystals under high pressure: An ab initio investigation Phys. Rev. B 67235321

[87] Yang S-C, Graupner W, Guha S, Puschnig P, Martin C, Chandrasekhar H R, Chandrasekhar M, Leising G, Ambrosch-Draxl C and Scherf U 2000 Geometrydependent electronic properties of highly fluorescent conjugated molecules Phys. Rev. Lett. 852388

[88] Hummer K, Puschnig P and Ambrosch-Draxl C 2004 Lowest optical excitations in molecular crystals: Bound excitons versus free electron-hole pairs in anthracene Phys. Rev. Lett. 92147402
Guha S, Chandrasekhar M, Scherf U and Knaapila M 2011 Tuning structural and optical properties of blue-emitting polymeric semiconductors Phys. Status Solidi B 2481083

Paudel K, Knoll H, Chandrasekhar M and Guha S 2010 Tuning intermolecular interactions in dioctyl-substituted polyfluorene via hydrostatic pressure J. Phys. Chem. A 114 4680

Murnaghan F D 1937 Finite deformations of an elastic solid Amer. J. Math. 59235

Heimel G, Hummer K, Ambrosch-Draxl C, Chunwachirasiri W, Winokur M J, Hanfland M, Oehzelt M, Aichholzer A and Resel R 2006 Phase transition and electronic properties of fluorene: A joint experimental and theoretical high-pressure study Phys. Rev. B 73024109

Schmidtke J P, Kim J-S, Gierschner J, Silva C and Friend R H 2007 Optical spectroscopy of a polyfluorene copolymer at high pressure: Intra- and intermolecular interactions Phys. Rev. Lett. 99167401

[94] Huang Y-S, Gierschner J, Schmidtke J P, Friend R H and Beljonne D 2011 Tuning interchain and intrachain interactions in polyfluorene copolymers Phys. Rev. B 84 205311

[95] Schmidtke J P, Friend R H and Silva C 2008 Tuning interfacial charge-transfer excitons at polymer-polymer heterojunctions under hydrostatic pressure Phys. Rev. Lett. 100157401

Bliznyuk V N, Carter S A, Scott J C, Klärner G, Miller R D and Miller D C 1999 Electrical and photoinduced degradation of polyfluorene based films and light-emitting devices Macromolecules 32361

[97] Weinfurtner K-H, Fujikawa H, Tokito S and Taga Y 2000 Highly efficient pure blue electroluminescence from polyfluorene: Influence of the molecular weight distribution on the aggregation tendency Appl. Phys. Lett. 762502

[98] List E J W, Guentner R, Scanducci de Freitas P and Scherf U 2002 The effect of keto defect sites on the emission properties of polyfluorene-type materials Adv. Mater. 14 374

[99] Arif M, Mukhopadhyay S, Ramasesha S and Guha S 2009 The role of triplet states in the emission mechanism of polymer light-emitting diodes Europhys. Lett. 8757008

[100] Mukhopadhyay S, Ramasesha S and Guha S 2010 Role of the triplet state in the green emission peak of polyfluorene films: A time evolution study J. Chem. Phys. 132044104

[101] Martin C M, Guha S, Chandrasekhar M, Chandrasekhar H R, Guentner R, Scanduicci de Freitas P and Scherf U 2003 Hydrostatic pressure dependence of the luminescence and raman frequencies in polyfluorene Phys. Rev. B 68115203

Sinha S, Rothe C, Güntner R, Scherf U and Monkman A P 2003 Electrophosphorescence and delayed electroluminescence from pristine polyfluorene thin-film devices at low temperature Phys. Rev. Lett. 90127402

Köhler A and Bässler H 2009 Triplet states in organic semiconductors Mater. Sci. Eng. R-Rep. 6671

Cardona M (1969) Modulation spectroscopy: Academic Press)

[105] Aryanpour K, Sheng C X, Olejnik E, Pandit B, Psiachos D, Mazumdar S and Vardeny Z V 2011 Evidence for excimer photoexcitations in an ordered $\pi$-conjugated polymer film Phys. Rev. B 83

[106] Botta C, Luzzati S, Tubino R, Bradley D D C and Friend R H 1993 Photoinduced absorption of polymer solutions 


\section{Phys. Rev. B 4814809}

[107] Samiullah M, Moghe D, Scherf U and Guha S 2010 Diffusion length of triplet excitons in organic semiconductors Phys. Rev. B 82

[108] Chandrasekhar M, Guha S and Graupner W 2001 Squeezing organic conjugated molecules-what does one learn? Adv. Mater. 13613

[109] Lupton J M, Pogantsch A, Piok T, List E J W, Patil S and Scherf U 2002 Intrinsic room-temperature electrophosphorescence from a $\$ \$ \backslash p i \$\}$-conjugated polymer Phys. Rev. Lett. 89167401

[110] Reufer M, Lagoudakis P G, Walter M J, Lupton J M, Feldmann J and Scherf U 2006 Evidence for temperatureindependent triplet diffusion in a ladder-type conjugated polymer Phys. Rev. B 74241201

[111] Paudel K, Moghe D, Chandrasekhar M, Yu P, Ramasesha S, Scherf U and Guha S 2013 Pressure dependence of singlet and triplet excitons in amorphous polymer semiconductors Europhys. Lett. 10427008

[112] Albert-Seifried S, Hodgkiss J M, Laquai F, Bronstein H A, Williams C K and Friend R H 2010 Pressure-induced delocalization of photoexcited states in a semiconducting polymer Phys. Rev. Lett. 105195501

[113] Chauvet O, Sienkiewicz A, Forro L and Zuppiroli L 1995 High-pressure electron-spin dynamics in disordered conducting polymers Phys. Rev. B 52 R13118

[114] Menéndez J (2000) Characterization of bulk semiconductors using raman spectroscopy (Raman scattering in materials science vol 42: Springer Berlin Heidelberg), pp 55

[115] Hsu S (2000) Raman spectroscopic studies of polymer structure (Raman scattering in materials science vol 42: Springer Berlin Heidelberg), pp 369

[116] Grüneisen E 1908 Relation between compressibility, thermal expansion, atom volume and atomic heat of the metals Annalen der Physik 26394

[117] Lucazeau G 2003 Effect of pressure and temperature on raman spectra of solids: Anharmonicity J. Raman Spectrosc. 34478

[118] Flores J J and Chronister E L 1996 Pressuredependentraman shifts of molecular vibrations in poly(methyl methacrylate) and polycarbonate polymers $J$. Raman Spectrosc. 27149

[119] Ulrich C, Anastassakis E, Syassen K, Debernardi A and Cardona M 1997 Lifetime of phonons in semiconductors under pressure Phys. Rev. Lett. 781283

[120] Heimel G, Somitsch D, Knoll P and Zojer E 2002 Ab initio study of vibrational anharmonic coupling effects in oligo(para-phenylenes) J. Chem. Phys. 11610921

[121] Cuff L and Kertesz M 1994 Ab initio oligomer approach to vibrational spectra of polymers: Comparison of helical and planar poly(p-phenylene) Macromolecules 27762

[122] Cuff L, Cui C and Kertesz M 1994 Role of charge transfer and quinonoid structure in the raman spectrum of doped poly(p-phenylene) J. Am. Chem. Soc. 1169269

[123] Louarn G, Athouël L, Froyer G, Buisson J P and Lefrant S 1993 Optical characterization of parasexiphenyl : A model compound of polyparaphenylene Synth. Met. 574762

[124] Leising G, Verdon T, Louarn G and Lefrant S 1991 Electronic properties of polyparaphenylene prepared by a precursor route Synth. Met. $\mathbf{4 1} 279$

[125] Albrecht A C 1961 On the theory of raman intensities $J$.
Chem. Phys. 341476

Albrecht A C and Hutley M C 1971 On the dependence of vibrational raman intensity on the wavelength of incident light J. Chem. Phys. 554438

Heimel G, Somitsch D, Knoll P, Brédas J-L and Zojer E 2005 Effective conjugation and raman intensities in oligo(para-phenylene)s: A microscopic view from firstprinciples calculations J. Chem. Phys. 122114511

[128] Volz C, Arif M and Guha S 2007 Conformations in dioctyl substituted polyfluorene: A combined theoretical and experimental raman scattering study J. Chem. Phys. 126

Adil D and Guha S 2013 Surface-enhanced raman spectroscopic studies of the au-pentacene interface: A combined experimental and theoretical investigation $J$. Chem. Phys. 139044715

[130] Martin C M, Cai Q, Guha S, Graupner W, Chandrasekhar M and Chandrasekhar H R 2004 Raman modes in oligophenyls under hydrostatic pressure Phys. Status Solidi B 2413339

Zhuravlev K K and McCluskey M D 2001 Flattening of organic molecules under pressure J. Chem. Phys. 1145465

Zhuravlev K K and McCluskey M D 2004 Conformation of p-terphenyl under hydrostatic pressure J. Chem. Phys. 120 1841

(1961) Landolt-börnstein physikalisch-chemische tabellen

$$
\text { Springer-Verlag) }
$$

[134] Weinstein B A and Piermarini G J 1975 Raman scattering and phonon dispersion in si and gap at very high pressure Phys. Rev. B 121172

Akishige Y, Nakahara J and Sawaguchi E 1991 Hydrostatic pressure effects on the raman scattering in hexagonal type batio3 J. Phys. Soc. Jpn. 601115

Loridant S and Lucazeau G 1999 High-pressure raman study of the perovskite baceo3 J. Raman Spectrosc. 30485

Slack G A and Bartram S F 1975 Thermal expansion of some diamondlike crystals J. Appl. Phys. 4689

Franco O, Orgzall I, Regenstein W and Schulz B 2006 Structural and spectroscopical study of a 2,5-diphenyl1,3,4-oxadiazole polymorph under compression J. Phys.: Condens. Matter 181459

Chopelas A, Boehler R and Ko T 1994 Thermodynamics and behavior of $\gamma$-mg2sio4 at high pressure: Implications for mg2sio4 phase equilibrium Phys. Chem. Miner. 21351

[140] Qin Z-X, Chen X-J, Zhang C, Tang L-Y, Zhong G-H, Lin H-Q, Meng Y and Mao H-K 2013 Vibrational and structural properties of tetramethyltin under pressure $J$. Chem. Phys. 138024307

[141] Chen P-N, Zha C-S, Chen X-J, Shu J, Hemley R J and Mao H-k 2011 Raman study of phase transitions in compressed methane using moissanite anvil cells Phys. Rev. B 84 104110

Hofmeister A M and Mao H-k 2002 Redefinition of the mode grüneisen parameter for polyatomic substances and thermodynamic implications Proc. Natl. Acad. Sci. U. S. A. 99559

[143] Proctor J E, Gregoryanz E, Novoselov K S, Lotya M, Coleman J N and Halsall M P 2009 High-pressure raman spectroscopy of graphene Phys. Rev. B $\mathbf{8 0} 073408$

[144] Nayak A P, Pandey T, Voiry D, Liu J, Moran S T, Sharma A, Tan C, Chen C-H, Li L-J, Chhowalla M, Lin J-F, Singh A K and Akinwande D 2015 Pressure-dependent optical 
and vibrational properties of monolayer molybdenum [164] disulfide Nano Lett. 15346

[145] Negri F and Zgierski M Z 1992 Franck-condon analysis of the $\mathrm{s} 0 \rightarrow \mathrm{t} 1$ absorption and phosphorescence spectra of biphenyl and bridged derivatives The Journal of Chemical Physics 977124

[146] Tsoi W C and Lidzey D G 2008 Raman spectroscopy of fluorene oligomers in the $\alpha-\beta$ - and $\gamma$-phases $J$. Phys.: Condens. Matter 20125213

[147] Chandrasekhar M, Renucci J B and Cardona M 1978 Effects of interband excitations on raman phonons in heavily doped n-si Phys. Rev. B 171623

[148] Pintschovius L, Vergés J A and Cardona M 1982 Selfenergies of phonons in heavily doped \$n\$- and \$p\$-type silicon Phys. Rev. B 265658

[149] Klein M V (1983) Electronic raman scattering (Light scattering in solids $i$ vol 8: Springer Berlin Heidelberg), pp 147

[150] Fano U 1961 Effects of configuration interaction on intensities and phase shifts Physical Review 1241866

[151] Yoon D, Jeong D, Lee H-J, Saito R, Son Y-W, Lee H C and Cheong H 2013 Fano resonance in raman scattering of graphene Carbon $\mathbf{6 1} 373$

[152] Hasdeo E H, Nugraha A R T, Dresselhaus M S and Saito R 2014 Breit-wigner-fano line shapes in raman spectra of graphene Phys. Rev. B 90245140

[153] Cardona M (2002) Phonons, electrons, and electronphonon interaction: Semiconductors and high-t $c$ superconductors (Ultrafast dynamics of quantum systems vol 372: Springer US), pp 257

[154] Morandi V, Galli M, Marabelli F and Comoretto D 2009 Highly oriented poly(paraphenylene vinylene): Polarized optical spectroscopy under pressure Phys. Rev. B 79 045202

[155] Stepanyan R, Subbotin A, Knaapila M, Ikkala O and ten Brinke G 2003 Self-organization of hairy-rod polymers Macromolecules 363758

[156] Schatschneider B and Lian J J 2011 Simulated pressure response of crystalline indole J. Chem. Phys. 135164508

[157] Orgzall I, Emmerling F, Schulz B and Franco O 2008 Highpressure studies on molecular crystals - relations between structure and high-pressure behavior J. Phys. : Condes. Matter 20295206

[158] Schiødt N C, Bjørnholm T, Bechgaard K, Neumeier J J, Allgeier C, Jacobsen C S and Thorup N 1996 Structural, electrical, magnetic, and optical properties of bis-benzene1,2-dithiolato-au(iv) crystals Phys. Rev. B 531773

[159] Oehzelt M, Heimel G, Resel R, Puschnig P, Hummer K, Ambrosch-Draxl C, Takemura K and Nakayama A 2003 High pressure $\mathrm{x}$-ray study on anthracene J. Chem. Phys. 1191078

[160] Oehzelt M, Aichholzer A, Resel R, Heimel G, Venuti E and Della Valle R G 2006 Crystal structure of oligoacenes under high pressure Phys. Rev. B 74104103

[161] Puschnig P, Ambrosch-Draxl C, Heimel G, Zojer E, Resel R, Leising G, Kriechbaum M and Graupner W 2001 Pressure studies on the intermolecular interaction in biphenyl Synth. Met. 116327

[162] Belsky V K, Zavodnik V E and Vozzhenikov V M 1984 Fluorene, c13h10 Acta Cryst. C $\mathbf{4 0} 1210$

[163] McFarlane S, McDonald R and Veinot J G C 2005 9,9-di-noctyl-9h-fluorene Acta Cryst. C 61 o671
Ma J, Fischer J E, Cao Y and Heeger A J 1992 X-ray structural study of trans-polyacetylene at high pressure Solid State Comm. 83395

Papanek P and Fischer J E 1993 Molecular-dynamics simulation of crystalline trans-polyacetylene Phys. Rev. B 4812566

Matsushita A, Akagi K, Liang T-S and Shirakawa H 1999 Effects of pressure on the electrical resistivity of iodine doped polyacetylene Synth. Met. 101447

Zhu Q and Fischer J E 1992 Crystal structure of polyacetylene revisited: An x-ray study Solid State Comm. 83179

Mårdalen J, Cerenius Y and Häggkvist P 1995 The crystalline structure of poly(3-octylthiophene) at high pressure J. Phys.: Condens. Matter 73501

Mårdalen J, Samuelsen E J, Konestabo O R, Hanfland M and Lorenzen M 1998 Conducting polymers under pressure: Synchrotron x-ray determined structure and structure related properties of two forms of poly(octylthiophene) J. Phys.: Condens. Matter 107145

Winokur M J (1998) Structural studies of conducting polymers (Handbook of conducting polymers) (New York: Marcel Dekker), pp 707

Prosa T J, Winokur M J, Moulton J, Smith P and Heeger A J 1995 X-ray-diffraction studies of the three-dimensional structure with iodine-intercalated poly(3-octylthiophene) Phys. Rev. B 51159

Corish J, Morton-Blake D A, Bénière $\mathrm{F}$ and Lantoine $\mathrm{M}$ 1996 Interaction of side-chains in poly(3-alkylthiophene) lattices J. Chem. Soc., Faraday Trans. 92671

O'Dwyer S, Xie H, Corish J and Morton-Blake D A 2001 An atomistic simulation of the effect of pressure on conductive polymers J. Phys.: Condens. Matter 132395

Agostinelli T, Lilliu S, Labram J G, Campoy-Quilles M, Hampton M, Pires E, Rawle J, Bikondoa O, Bradley D D C, Anthopoulos T D, Nelson J and Macdonald J E 2011 Realtime investigation of crystallization and phase-segregation dynamics in p3ht:Pcbm solar cells during thermal annealing Adv. Funct. Mater. 211701

Paudel K, Chandrasekhar M, Scherf U, Preis E and Guha S 2011 High-pressure optical studies of donor-acceptor polymer heterojunctions Phys. Rev. B 84205208

Noguchi Y, Saeki A, Fujiwara T, Yamanaka S, Kumano M, Sakurai T, Matsuyama N, Nakano M, Hirao N, Ohishi Y and Seki S 2015 Pressure modulation of backbone conformation and intermolecular distance of conjugated polymers toward understanding the dynamism of $\pi$ figuration of their conjugated system J. Phys. Chem. B 119 7219

[177] Gitsas A, Floudas G and Wegner G 2004 Effects of temperature and pressure on the stability and mobility of phases in rigid rod poly(p-phenylenes) Phys. Rev. E 69 041802

Takizawa K, Fukudome H, Kozaki Y and Ando S 2014 Pressure-induced changes in crystalline structures of polyimides analyzed by wide-angle x-ray diffraction at high pressures Macromolecules 473951

[179] Takizawa K, Wakita J, Kakiage M, Masunaga H and Ando S 2010 Molecular aggregation of polyimide films at very high pressure analyzed by synchrotron wide-angle x-ray diffraction Macromolecules 432115

Takizawa K, Wakita J, Azami S and Ando S 2011 Relationship between molecular aggregation structures and 
optical properties of polyimide films analyzed by synchrotron wide-angle $\mathrm{x}$-ray diffraction, infrared absorption, and uv/visible absorption spectroscopy at very high pressure Macromolecules $\mathbf{4 4} 349$

[181] Takizawa K, Wakita J, Sekiguchi K and Ando S 2012 Variations in aggregation structures and fluorescence properties of a semialiphatic fluorinated polyimide induced by very high pressures Macromolecules 454764

[182] Knaapila M, Stepanyan R, Haase D, Carlson S, Torkkeli M, Cerenius Y, Scherf U and Guha S 2010 Evidence for structural transition in hairy-rod poly[9,9-bis(2ethylhexyl)fluorene] under high pressure conditions Phys. Rev. E 82051803

[183] Landau L D and Lifshits E M (1980) Statistical physics (Oxford: Pergamon)

[184] Brinkmann M 2007 Directional epitaxial crystallization and tentative crystal structure of poly(9,9'-di-n-octyl-2,7fluorene) Macromolecules 407532

[185] Grell M, Bradley D D C, Ungar G, Hill J and Whitehead K S 1999 Interplay of physical structure and photophysics for a liquid crystalline polyfluorene Macromolecules 325810

[186] Knaapila M, Konôpková Z, Torkkeli M, Haase D, Liermann H-P, Guha S and Scherf U 2013 Structural study of helical polyfluorene under high quasihydrostatic pressure Phys. Rev. E 87022602

[187] Knaapila M, Torkkeli M, Konôpková Z, Haase D, [195] Liermann H-P, Scherf U and Guha S 2013 Measuring structural inhomogeneity of conjugated polymer at high pressures up to 30 gpa Macromolecules 468284
[188] McFarlene S, McDonald R and Veinot J G C 2005 9,9-di-noctyl-9h-fluorene Acta Cryst. C 61 o671

[189] Martin C M, Guha S, Chandrasekhar M, Chandrasekhar H R, Guentner R, Scanduicci de Freitas P and Scherf U 2003 Hydrostatic pressured dependence of the luminescence and raman frequencies in polyfluorene Phys. Rev. B 68115203

[190] Klotz S, Chervin J-C, Munsch P and Le Marchand G 2009 Hydrostatic limits of 11 pressure transmitting media $J$. Phys. D: Appl. Phys. 42075413

[191] Shi W, Ye J, Checkelsky J G, Terakura C and Iwasa Y 2014 Transport properties of polymer semiconductor controlled by ionic liquid as a gate dielectric and a pressure medium Adv. Funct. Mater. 242005

Xiao H, An Q, Goddard W A, Liu W-G and Zybin S V 2013 Formation of the -n(no)n(no)- polymer at high pressure and stabilization at ambient conditiosn Proc. Natl. Acad. Sci. 1105321

[193] Barraud C, Seneor P, Mattana R, Fusil S, Bouzehouane K, Deranlot C, Graziosi P, Hueso L, Bergenti I, Dediu V, Petroff F and Fert A 2010 Unravelling the role of the interface for spin injection into organic semiconductors Nat. Phys. 6

[194] Hu B and Wu Y 2007 Tuning magnetoresistance between positive and negative values in organic semiconductors Nat. Mater. 6985

Wohlgenannt M 2012 Organic magnetoresistance and spin diffusion in organic semiconductor thin film devices Phys. Status Solidi RRL 6229 